Supporting Information for:

\title{
Tris(pyrazolyl)borate Copper Hydroxide Complexes Featuring Tunable Intramolecular H-bonding
}

Evan J. Gardner, Caitlyn R. Cobb, Jeffery A. Bertke, and Timothy H. Warren*

Department of Chemistry, Georgetown University, Box 571227, Washington, DC 20057, USA

\section{Contents}

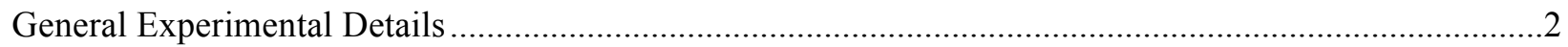

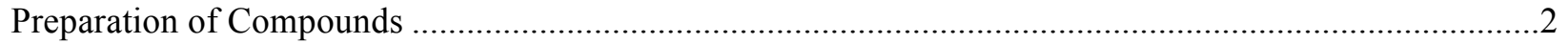

Synthesis and Characterization of Methyl 5-(trifluoromethyl)picolinate (1a) ...................................2

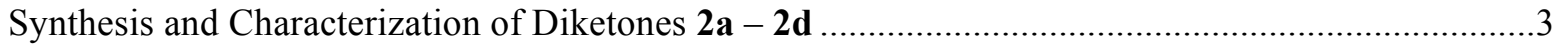

Synthesis and Characterization of Pyridine-pyrazoles ${ }^{\text {pyMe }} \mathrm{PzH}(\mathbf{3 a}-\mathbf{3 d})$..........................................

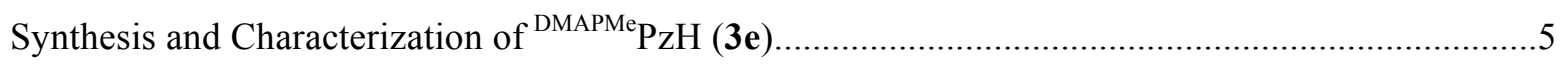

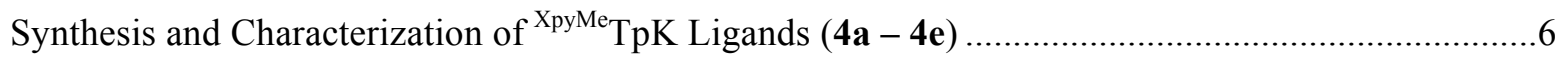

Synthesis and Characterization of ${ }^{\mathrm{XpyMe}} \mathrm{TpCuOAc}$ Complexes $(\mathbf{5 a}-\mathbf{5 e})$...........................................

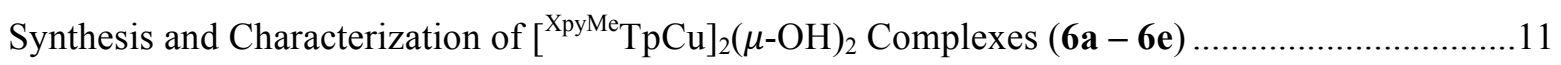

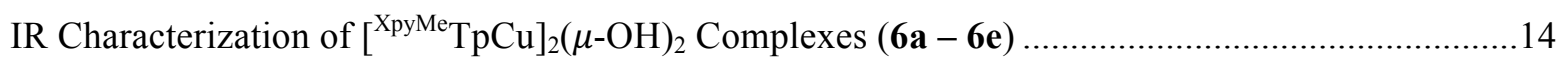

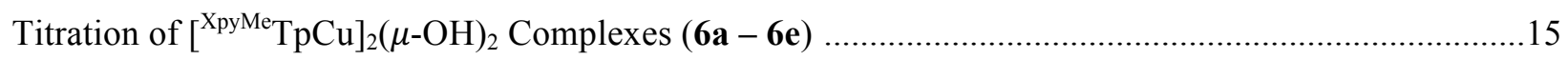

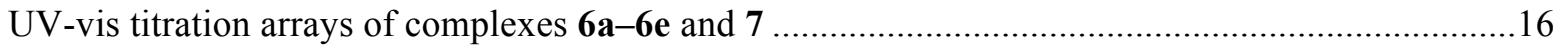

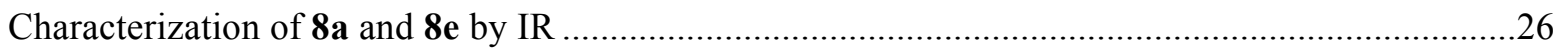

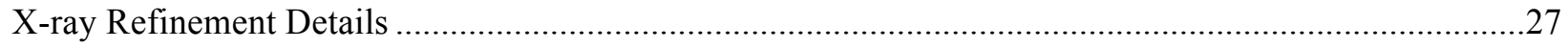

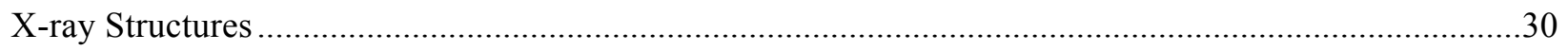

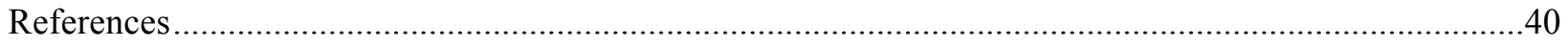




\section{General Experimental Details}

All experiments were conducted in a dry dinitrogen atmosphere using an MBraun glovebox and/or standard Schlenk techniques unless otherwise noted. Dry dicholoromethane (DCM), diethyl ether, tetrahydrofuran (THF), methanol (MeOH), and pentane were purchased from Acros and were stored over activated 4A molecular sieves under dinitrogen. The 4A molecular sieves were activated prior to use in vacuo at $200{ }^{\circ} \mathrm{C}$ for $24 \mathrm{~h}$. Deuterated solvents were sparged with dinitrogen, stored over $4 \mathrm{~A}$ molecular sieves, and stored under a dinitrogen atmosphere. ${ }^{1} \mathrm{H},{ }^{13} \mathrm{C}$, and ${ }^{19} \mathrm{~F}$ NMR spectra were recorded on either a Varian $300 \mathrm{MHz}$ or $400 \mathrm{MHz}$ spectrometer (300 or 400, 75.4 or $100.4,376.1$ or $282.3 \mathrm{MHz}$ respectively) at Georgetown University. All NMR spectra were collected at room temperature and were indirectly referenced to residual solvent signals, TMS, or fluorobenzene as internal standards. UV-vis spectra were measured at Georgetown on a Varian Cary 8454 or Cary 50 spectrometer each fitted with a Unisoku Unispek Coolspeck variable temperature cell holder. IR vibrational spectra were taken on a Varian 3100 FTIR/ATR.

Anhydrous $\mathrm{Cu}(\mathrm{OAc})_{2}$ was purchased from Strem, $\mathrm{TMSCHN}_{2}$ and HOTf were purchased from SigmaAldrich and stored at $-40{ }^{\circ} \mathrm{C}$ in a glovebox prior to use. 5-(trifluoromethyl)pyridine-2-carboxylic acid (1a), methyl-2-pyrimidinecarboxylate (1b), and HOTf were purchased from Matrix Scientific and were purified via sublimation prior to reactions. Ethyl 2-picolinate (1c) and 4 -chloropyridine-2-carboxylic acid (1d), were purchased from TCI America and were used without further purification. 2,4,6-collidine, triethylamine $\left(\mathrm{Et}_{3} \mathrm{~N}\right)$, and 1,8-diazabicyclo[5.4.0]undec-7-ene (DBU) were purchased from TCI America and stored in the glovebox over activated $4 \mathrm{~A}$ sieves prior to use. The ${ }^{\mathrm{iPr} 2} \mathrm{TpK}$ starting material was prepared according to literature procedures ${ }^{1}$ and the preparation of ${ }^{\mathrm{XpyMe}} \mathrm{TpCuOAc}(\mathbf{5 a}-\mathbf{5 e})$ and $\left[{ }^{\mathrm{XpyMe}} \mathrm{TpCu}\right]_{2}(\mu-\mathrm{OH})_{2}(\mathbf{6 a}-\mathbf{6 e})$ were synthesized using procedures adapted from the preparation of ${ }^{\mathrm{R}} \mathrm{TpCuOAc}$ and $\left[{ }^{\mathrm{iPr} 2} \mathrm{TpCu}\right]_{2}(\mu-\mathrm{OH})_{2}$, respectively. ${ }^{2,3}$ The magnetic susceptibility of paramagnetic compounds was determined using an adapted Evans method ${ }^{4}$ using a coaxial NMR tube and ${ }^{19} \mathrm{~F}$ NMR as outlined by Ghosh and Darensbourg. ${ }^{5}$ The applied diamagnetic susceptibility corrections for 5a - 5e and 6a - 6e were calculated and were close to [-(mol.wt.)/2]/1000000 for each individual species. ${ }^{6}$ Elemental analyses were performed on a Perkin-Elmer PE4200 microanalyzer at Georgetown and HRMS samples analyses were performed at mass spectrometry facilities at the Indiana University.

\section{Preparations of Compounds}

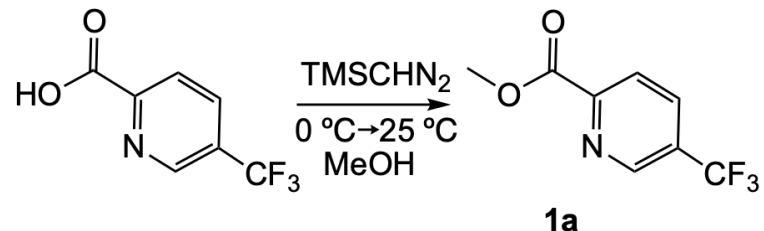

Scheme S1. Reaction of 5-(trifluoromethyl)picolinic acid with TMS diazomethane. 


\section{Synthesis of Methyl 5-(trifluoromethyl)picolinate (1a).}

In a foil wrapped $250 \mathrm{~mL}$ Schlenk flask, (2.325 g, $12.2 \mathrm{mmol})$ 5-(trifluoromethyl)picolinic acid was added to $(100 \mathrm{~mL})$ 50:50 solution of dry $\mathrm{MeOH}$ and $\mathrm{THF}$, chilled to $0{ }^{\circ} \mathrm{C}$, and stirred under dynamic $\mathrm{N}_{2}$ flow. A $2 \mathrm{M}$ solution of $\mathrm{TMSCHN}_{2}$ in $\mathrm{Et}_{2} \mathrm{O}$ was slowly added dropwise $(6.7 \mathrm{~mL}, 13.4 \mathrm{mmol})$, resulting in vigorous gas evolution. The reaction was stirred at high velocity for $2 \mathrm{~h}$ and allowed to gradually warm to room temperature followed by stirring for an additional $14 \mathrm{~h}$. The solution was concentrated to dryness and solids were purified via sublimation at $80{ }^{\circ} \mathrm{C}$ under dynamic vacuum for $6 \mathrm{~h}$. Light grey crystals were collected without further purification. (1.802 g, $8.7 \mathrm{mmol}, 72 \%$ yield). Characterization data matched that from materials purchased from Matrix Scientific as well as that in a literature report. ${ }^{7}$

\section{General Synthesis of Diketones 2a-2d}

Syntheses of diketones 2a - 2d uniformly follow an adapted procedure. ${ }^{8}$ Each respective ester $(\mathbf{1 a}-\mathbf{1 d})$ was added to a 50:50 mixture of THF and $\mathrm{Et}_{2} \mathrm{O}$ containing a slight excess of acetone under an $\mathrm{N}_{2}$ atmosphere. This solution was chilled to $-45{ }^{\circ} \mathrm{C}$ and $\mathrm{KH}$ was added gradually. After stirring at room temperature for $4 \mathrm{~h}$, the solution was neutralized by addition of $1 \mathrm{M} \mathrm{NH}_{4} \mathrm{Cl}$ (aq) adjusted to be equimolar to the $\mathrm{KH}$ used. Organics were extracted with $3 \times 50 \mathrm{~mL}$ portions of $\mathrm{Et}_{2} \mathrm{O}$ and were dried over anhydrous $\mathrm{MgSO}_{4}$. Products were purified using a silica flash column (1:5 EtOAc : hexane).

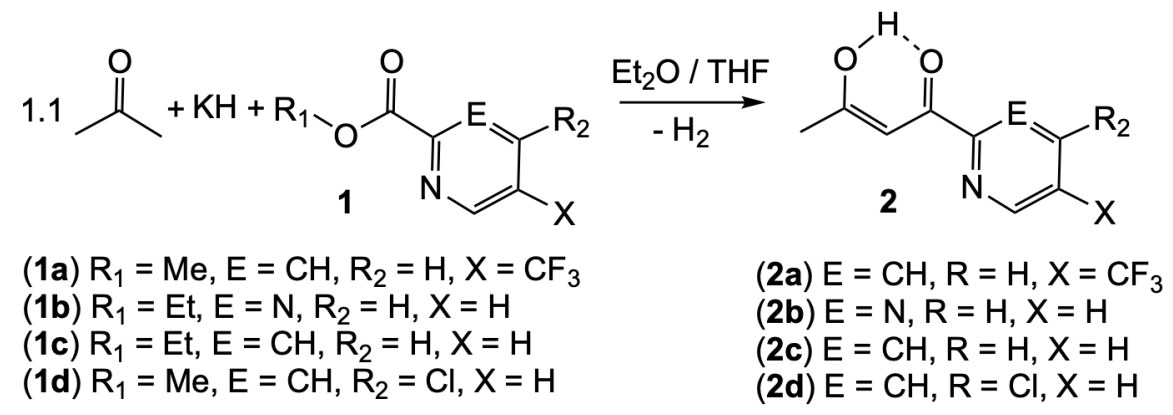

Scheme S2. General condensation reaction to form diketones $\mathbf{2 a - 2 d}$.

1-(5-(trifluoromethyl)pyridin-2-yl)butane-1,3-dione (2a). 1a (5.769 g, $28.1 \mathrm{mmol})$, dry acetone (2.3 $\mathrm{mL}$, 31.0 mmol, 1.1 equiv.), and KH (1.131 g, $28.2 \mathrm{mmol}, 1$ equiv.) yielded a light yellow powder of 2a (4.202 g, $18.2 \mathrm{mmol}, 65 \%) .{ }^{1} \mathrm{H}$ NMR $\left(\mathrm{CDCl}_{3}\right): \delta_{\mathrm{H}} 15.51(\mathrm{~s}, 1 \mathrm{H}), 8.87(\mathrm{~d}, J=1.6 \mathrm{~Hz}, 1 \mathrm{H}), 8.15(\mathrm{~d}, 8.2 \mathrm{~Hz}$, $1 \mathrm{H}), 8.04(\mathrm{dd}, J=8.2,1.6 \mathrm{~Hz}, 1 \mathrm{H}), 6.84(\mathrm{~s}, 1 \mathrm{H}), 2.24(\mathrm{~s}, 1 \mathrm{H}) \cdot{ }^{13} \mathrm{C} \mathrm{NMR}\left(\mathrm{CDCl}_{3}\right): \delta_{\mathrm{C}} 196.5,178.3$, $155.3(\mathrm{q}, J=1.8 \mathrm{~Hz}), 146.3(\mathrm{q}, J=4.0 \mathrm{~Hz}), 134.4(\mathrm{q}, J=3.6 \mathrm{~Hz}), 128.5(\mathrm{q}, J=33.3 \mathrm{~Hz}), 123.3(\mathrm{q}, J=$ 
272.6 Hz), 121.7, 98.2, 26.5. ${ }^{19} \mathrm{~F}$ NMR $\left(\mathrm{CDCl}_{3}\right): \delta_{\mathrm{F}}$-62.7. Anal. Calcd. for $\mathrm{C}_{10} \mathrm{H}_{8} \mathrm{~F}_{3} \mathrm{NO}_{2}(\mathbf{2 a}): \mathrm{C}, 52.01 ; \mathrm{H}$, 3.49; N, 6.06. Found: C, 52.01; H, 3.60; N, 6.11.

1-(pyrimidin-2-yl)butane-1,3-dione (2b). $1 \mathbf{b}(9.564 \mathrm{~g}, 62.9 \mathrm{mmol})$, dry acetone (5.6 mL, $75.6 \mathrm{mmol}, 1.1$ equiv.), and KH (2.800 g, 69.8 mmol, 1.1 equiv.) yielded 2b (6.875 g, 41.9 mmol, 67 \%). ${ }^{1} \mathrm{H}$ NMR $\left(\mathrm{CDCl}_{3}\right): \delta_{\mathrm{H}} 15.36(\mathrm{~s}, 1 \mathrm{H}), 8.89(\mathrm{~d}, J=4.9 \mathrm{~Hz}, 2 \mathrm{H}), 7.39(\mathrm{t}, J=4.9 \mathrm{~Hz}, 1 \mathrm{H}), 6.90(\mathrm{~s}, 1 \mathrm{H}), 2.30(\mathrm{~s}, 3 \mathrm{H})$.

${ }^{13} \mathrm{C}$ NMR: $\delta_{\mathrm{C}} 198.2,175.3,159.9,157.3,121.9,100.1,27.0$. One ${ }^{13} \mathrm{C}$ resonance was not observed. Anal. Calcd. for $\mathrm{C}_{8} \mathrm{H}_{8} \mathrm{~N}_{2} \mathrm{O}_{2}(2 \mathbf{b})$ : C, 58.53; H, 4.91; N, 17.06. Found: C, 58.49; H, 5.04; N, 16.93 .

1-(pyridin-2-yl)butane-1,3-dione (2c). 1c $(10.0 \mathrm{~mL}, 74 \mathrm{mmol})$, dry acetone $(5.98 \mathrm{~mL}, 81 \mathrm{mmol}, 1.1$ equiv.), and KH (3.001 g, $74.8 \mathrm{mmol}, 1$ equiv.) yielded colorless crystals of 2c (8.369 g, $51.3 \mathrm{mmol}$, $69 \%$ ). Compound characterization data matched literature reports. ${ }^{8}$

1-(4-chloropyridin-2-yl)butane-1,3-dione (2d). 1d (5.025 g, $29.3 \mathrm{mmol})$, acetone (2.4 mL, $32.4 \mathrm{mmol}, 1.1$ equiv.), and KH (1.178 g, $29.3 \mathrm{mmol}, 1$ equiv.) yielded $2 \mathrm{~d}$ (4.516 g, $22.9 \mathrm{mmol}, 78 \%) .{ }^{1} \mathrm{H} \mathrm{NMR}\left(\mathrm{CDCl}_{3}\right)$ : $\delta_{\mathrm{H}} 15.57(\mathrm{~s}, 1 \mathrm{H}), 8.55(\mathrm{dd}, J=5.20,0.38 \mathrm{~Hz}, 1 \mathrm{H}), 8.08(\mathrm{dd}, J=2.07,0.38 \mathrm{~Hz}, 1 \mathrm{H}), 7.42(\mathrm{dd}, J=5.20$, $2.07 \mathrm{~Hz}, 1 \mathrm{H}), 6.81(\mathrm{~s}, 1 \mathrm{H}), 2.25$ (s, $3 \mathrm{H}) .{ }^{13} \mathrm{C}$ NMR: $\delta_{\mathrm{C}} 195.5,179.5,153.8,150.2,145.6,126.4,122.7$, 97.8, 26.3. Anal. Calcd. for $\mathrm{C}_{9} \mathrm{H}_{8} \mathrm{ClNO}_{2}$ : C, 54.70; H, 4.08; N, 7.09. Found: C, 54.57; H, 4.06; N, 7.06.

\section{General Synthesis of Pyridine-pyrazoles $\left({ }^{\mathrm{pyMe}} \mathrm{PzH}\right) 3 \mathrm{a}-\mathbf{3 d}$}

Each diketone (2a - 2d) was dissolved in $150 \mathrm{~mL}$ of $\mathrm{MeOH}$ in a $250 \mathrm{~mL}$ round bottom flask charged a stir bar. $1.5-2$ equiv. anhydrous hydrazine was added to this mixture slowly at room temperature and the reaction was left to stir for $4 \mathrm{~h}$. The solution was then concentrated in vacuo to dryness yielding pyrazoles (3a-3d), typically without need for further purification.
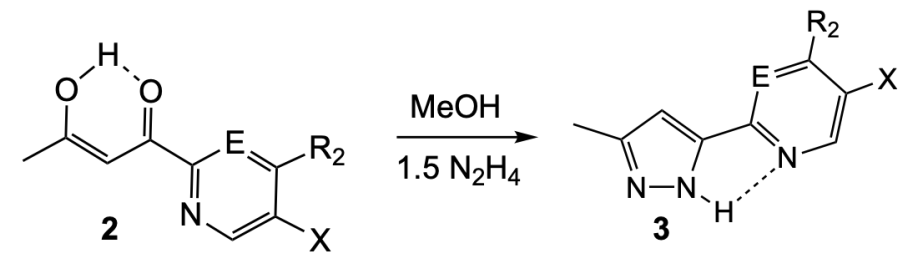

$$
\begin{aligned}
& \text { (2a) } E=C H, R=H, X=C_{3} \\
& \text { (2b) } E=N, R=H, X=H \\
& \text { (2c) } E=C H, R=H, X=H \\
& \text { (2d) } E=C H, R=C l, X=H
\end{aligned}
$$

(3a) $\mathrm{E}=\mathrm{CH}, \mathrm{R}=\mathrm{H}, \mathrm{X}=\mathrm{CF}_{3}$

(3b) $\mathrm{E}=\mathrm{N}, \mathrm{R}=\mathrm{H}, \mathrm{X}=\mathrm{H}$

(3c) $\mathrm{E}=\mathrm{CH}, \mathrm{R}=\mathrm{H}, \mathrm{X}=\mathrm{H}$

(3d) $\mathrm{E}=\mathrm{CH}, \mathrm{R}=\mathrm{Cl}, \mathrm{X}=\mathrm{H}$

Scheme S3. Condensation of diketones $2 \mathbf{a}-\mathbf{2 d}$ with hydrazine to form pyrazoles ${ }^{\mathrm{XpyMe}} \mathrm{PzH} \mathbf{3 a}-\mathbf{3 d}$. 
${ }^{\text {CF3pyMe }} \mathrm{PzH}$ (3a). A solid sample of $2 \mathbf{2 a}(4.202 \mathrm{~g}, 18.2 \mathrm{mmol})$ and $\mathrm{N}_{2} \mathrm{H}_{4}(0.87 \mathrm{~mL}, 27.8 \mathrm{mmol}, 1.5$ equiv.) yielded 3a as a light yellow powder $(3.896 \mathrm{~g}, 17.1 \mathrm{mmol}, 94 \%)$. ${ }^{1} \mathrm{H}$ NMR $\left(\mathrm{CDCl}_{3}\right): \delta_{\mathrm{H}} 8.85(\mathrm{~s}, 1 \mathrm{H}), 7.95$ $(\mathrm{dd}, \mathrm{J}=8.2,2.0 \mathrm{~Hz}, 1 \mathrm{H}), 7.88(\mathrm{~d}, \mathrm{~J}=8.2 \mathrm{~Hz}, 1 \mathrm{H})), 6.68(\mathrm{~s}, 1 \mathrm{H}), 2.40(\mathrm{~s}, 3 \mathrm{H}) .{ }^{13} \mathrm{C} \mathrm{NMR}\left(\mathrm{CDCl}_{3}\right): \delta_{\mathrm{C}}$ 153.6, 145.6, 146.6 (q, J = 4.2 Hz), 134.1 (q, J = 3.5 Hz), 125.3 (q, J = 33.0 Hz), 123.7 (q, J = 244.1 Hz), 119.5, 104.1, 12.4. ${ }^{19} \mathrm{~F}$ NMR $\left(\mathrm{CDCl}_{3}\right): \delta_{\mathrm{F}}-62.43(\mathrm{~s}, 3 \mathrm{~F})$. One ${ }^{13} \mathrm{C}$ resonance was not observed. Anal. Calcd. for $\mathrm{C}_{10} \mathrm{H}_{8} \mathrm{~F}_{3} \mathrm{~N}_{3}: \mathrm{C}, 52.87 ; \mathrm{H}, 3.55 ; \mathrm{N}, 18.50$. Found: C, 52.91; H, 3.52; N, 18.35.

${ }^{\text {pymMe }} \mathrm{PzH}$ (3b). A solid sample of $\mathbf{2 b}(2.878 \mathrm{~g}, 17.5 \mathrm{mmol})$ and $\mathrm{N}_{2} \mathrm{H}_{4}(0.83 \mathrm{~mL}, 26.4 \mathrm{mmol}, 1.5$ equiv.) yielded $\mathbf{3 b}$ as a tan powder $(2.774 \mathrm{~g}, 17.3 \mathrm{mmol}, 99 \%) .{ }^{1} \mathrm{H} \mathrm{NMR}\left(\mathrm{CDCl}_{3}\right): \delta_{\mathrm{H}} 8.75(\mathrm{~m}, 2 \mathrm{H}), 7.18(\mathrm{~m}, 1 \mathrm{H})$, $6.83(\mathrm{~s}, 1 \mathrm{H}), 2.38(\mathrm{~s}, 3 \mathrm{H}) .{ }^{13} \mathrm{C} \mathrm{NMR}\left(\mathrm{CDCl}_{3}\right): \delta_{\mathrm{C}} 158.4,157.5,148.4,144.0,119.5,106.0,13.2$. One ${ }^{13} \mathrm{C}$ resonance was not observed. Anal. Calcd. for $\mathrm{C}_{8} \mathrm{H}_{8} \mathrm{~N}_{4}$ : C, 59.99; H, 5.03; N, 34.98. Found: C, 60.05; H, $4.91 ; \mathrm{N}, 34.74$.

${ }^{p y M e} \mathrm{PzH}$ (3c). A solid sample of $\mathbf{2 c}(7.524 \mathrm{~g}, 46.2 \mathrm{mmol})$ and $\mathrm{N}_{2} \mathrm{H}_{4}(2.2 \mathrm{~mL}, 70 \mathrm{mmol}, 1.5$ equiv.) yielded $3 \mathrm{c}$ as an off-white powder $(6.324 \mathrm{~g}, 39.7 \mathrm{mmol}, 86 \%$ yield $)$. Compound characterization matched literature reports. ${ }^{8}$

${ }^{C l P y M e} \mathrm{PzH}$ (3d). A solid sample of $2 \mathbf{2 d}(4.516 \mathrm{~g}, 22.9 \mathrm{mmol})$ and $\mathrm{N}_{2} \mathrm{H}_{4}(1.0 \mathrm{~mL}, 31.9 \mathrm{mmol}, 1.5$ equiv.) yielded 3d (3.165 g, $16.3 \mathrm{mmol}, 72 \%)$. ${ }^{1} \mathrm{H}$ NMR $\left(\mathrm{CDCl}_{3}\right): \delta_{\mathrm{H}} 11.99$ (brs, $\left.1 \mathrm{H}\right), 8.49$ (d, $\left.5.4 \mathrm{~Hz}, 1 \mathrm{H}\right), 7.78$ (brs, $1 \mathrm{H}), 7.17(\mathrm{dd}, 5.4,2.0 \mathrm{~Hz}, 1 \mathrm{H}), 6.58(\mathrm{~s}, 1 \mathrm{H}), 2.32(\mathrm{~s}, 3 \mathrm{H}) .{ }^{13} \mathrm{C}$ NMR: $\delta_{\mathrm{C}} 150.4,144.9,122.8$, 120.3, 103.6, 77.2, 12.4. Two ${ }^{13} \mathrm{C}$ resonances were not observed. Anal. Calcd for $\mathrm{C}_{9} \mathrm{H}_{8} \mathrm{ClN}_{3}: \mathrm{C}, 55.83 ; \mathrm{H}$, 4.16; N, 21.70. Found: C, 55.68; H, 4.39; N, 21.45.

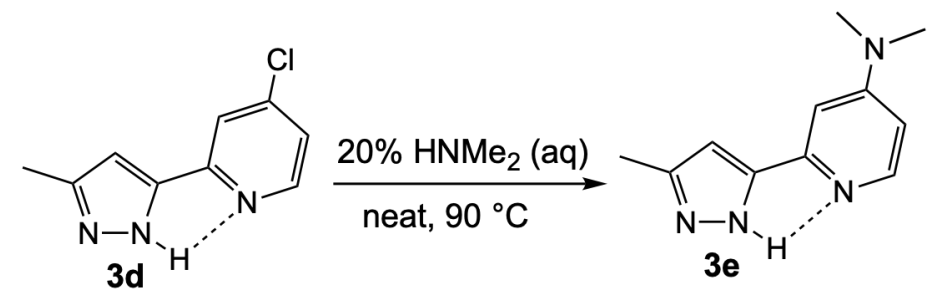

Scheme S4. Synthesis of ${ }^{\text {DMAPMe }} \mathrm{PzH}(\mathbf{3 e})$.

${ }^{D M A P y M e} \mathrm{PzH}$ (3e). A solid sample of $\mathbf{3 d}(1.750 \mathrm{~g}, 9.04 \mathrm{mmol})$ was added to a $350 \mathrm{~mL}$ thick walled glass pressure vessel charged with a stir bar. A large excess of $\mathrm{HNMe}_{2}$ was added (150 $\mathrm{mL}, 40 \% \mathrm{HNMe}_{2}(\mathrm{aq})$, $1.2 \mathrm{~mol})$. This mixture was gradually heated to $90{ }^{\circ} \mathrm{C}$ and left to react for $48 \mathrm{~h}$ to ensure complete conversion. The solution was gradually cooled to room temperature with a small amount of nucleation 
becoming visible. Subsequently, the mixture was cooled to chilled to $0{ }^{\circ} \mathrm{C}$ in an ice bath. The suspension 3e was filtered out over a fine frit and $\mathbf{3 e}$ was collected and dried on the frit completely in vacuo to yield a white powder (1.343 g, $6.6 \mathrm{mmol}, 73 \%)$. ${ }^{1} \mathrm{H}$ NMR (DMSO- $\left.d_{6}\right): \delta_{\mathrm{H}} 12.85$ (brs, $0.5 \mathrm{H}$ ), 12.58 (brs, $0.5 \mathrm{H}$ ), $8.11(\mathrm{~J}=5.9 \mathrm{~Hz}, 1 \mathrm{H}), 7.10$ (brs, $1 \mathrm{H}), 6.53(\mathrm{~s}, 1 \mathrm{H}), 6.52(\mathrm{dd}, \mathrm{J}=5.9,2.6 \mathrm{~Hz}, 1 \mathrm{H}), 2.98(\mathrm{~s}, 6 \mathrm{H}), 2.2(\mathrm{~s}, 3$ $\mathrm{H})$. Resonances slightly overlap, total integration of combined peaks adds to $2 \mathrm{H}$. ${ }^{13} \mathrm{C}$ NMR (DMSO- $d_{6}$ ): $\delta_{\mathrm{C}} 154.50,149.02,105.81,102.58,101.61,38.75$. Four ${ }^{13} \mathrm{C}$ resonances were not observed. Anal. Calcd. for $\mathrm{C}_{11} \mathrm{H}_{14} \mathrm{~N}_{4}(3 \mathbf{c})$ : C, 65.32; H, 6.98; N, 27.70. Found: C, 65.24; H, 7.31; N, 27.43.

\section{General Preparation of ${ }^{\mathrm{XpyMe}}$ TpK Ligands $4 \mathrm{a}-4 \mathrm{e}$}

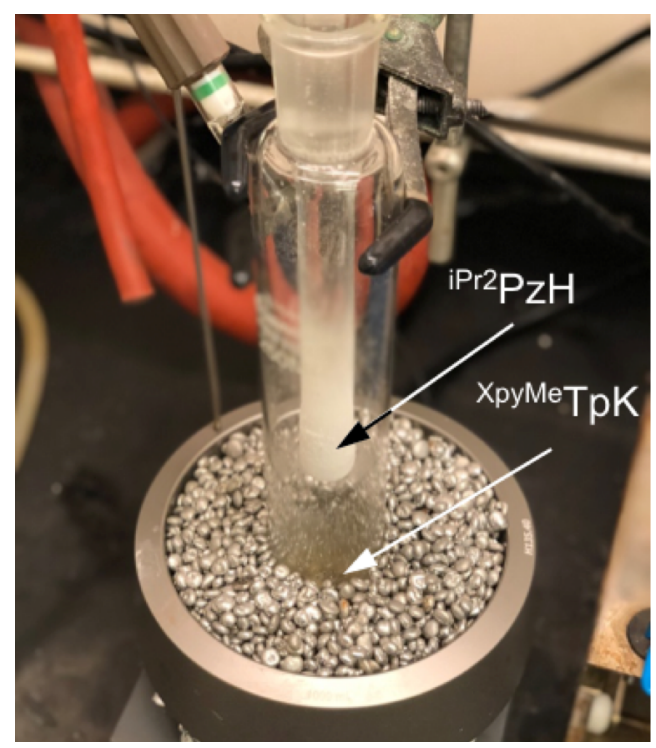

Figure S1. Sublimation apparatus used to generate ligands $\mathbf{3 a}-\mathbf{3 e}$ in a heated heat bead bath. The picture was taken at the end of the reaction. Arrows indicate sublimed ${ }^{\text {iPr2 }} \mathrm{PzH}$ onto water-chilled cold finger (top) and synthesized ${ }^{\mathrm{XpyMe}}$ TpK (bottom) at the bottom of the flask as a glass.

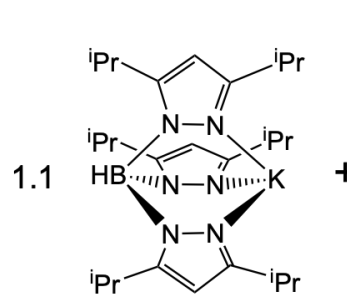

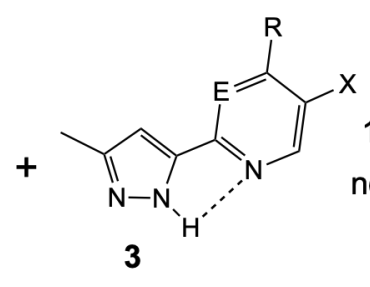

(3a) $\mathrm{E}=\mathrm{CH}, \mathrm{R}=\mathrm{H}, \mathrm{X}=\mathrm{CF}_{3}$

(3b) $\mathrm{E}=\mathrm{N}, \mathrm{R}=\mathrm{H}, \mathrm{X}=\mathrm{H}$

(3c) $\mathrm{E}=\mathrm{CH}, \mathrm{R}=\mathrm{H}, \mathrm{X}=\mathrm{H}$

(3e) $\mathrm{E}=\mathrm{CH}, \mathrm{R}=\mathrm{NMe}_{2}, \mathrm{X}=\mathrm{H}$

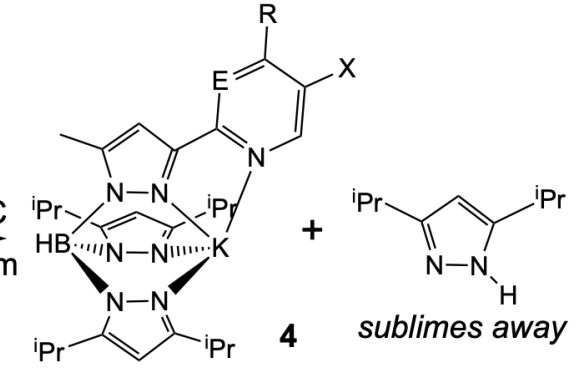

(4a) $\mathrm{E}=\mathrm{CH}, \mathrm{R}=\mathrm{H}, \mathrm{X}=\mathrm{CF}_{3}$

(4b) $E=N, R=H, X=H$

(4c) $\mathrm{E}=\mathrm{CH}, \mathrm{R}=\mathrm{H}, \mathrm{X}=\mathrm{H}$

(4e) $\mathrm{E}=\mathrm{CH}, \mathrm{R}=\mathrm{NMe}_{2}, \mathrm{X}=\mathrm{H}$

Scheme S5. The general synthesis of ${ }^{\mathrm{XpyMe}} \mathrm{TpK}$ ligands $4 \mathbf{a}-\mathbf{4 e}$. 


\section{Synthesis of ${ }^{\mathrm{XpyMe}}$ TpK Ligands $4 \mathrm{a}-4 \mathrm{e}$}

Reactions were carried out under ambient atmosphere in a $250 \mathrm{~mL}$ Schlenk tube charged with a stir bar. For $4 \mathbf{a}-4 \mathbf{c}^{\mathrm{iPr} 2} \mathrm{TpK}$ and ${ }^{\mathrm{Xpy}} \mathrm{PzH}$ were combined in a 1.1:1 ratio and stirred together under vacuum for 20 min at room temperature to form an evenly mixed powder. To increase yield, $\mathbf{4 e}$ was synthesized from a 1.3:1 mixture of ${ }^{\mathrm{iPr} 2} \mathrm{TpK}$ and ${ }^{\mathrm{DMAPMe}} \mathrm{PzH}$. In all cases water-chilled cold finger was inserted fed by flowing tap water was inserted. The vessel was evacuated again and gradually heated to $160{ }^{\circ} \mathrm{C}$ under dynamic vacuum. The reaction was allowed to proceed for $4 \mathrm{~h}$, resulting in noticeable ${ }^{\mathrm{iPr} 2} \mathrm{PzH}$ sublimation onto the cold finger (Scheme S1). See Figure S1 for a picture of the sublimation / reaction apparatus. Once gradually cooled to room temperature, the sublimed ${ }^{\mathrm{iPr} 2} \mathrm{PzH}$ was removed with the cold finger leaving behind the ${ }^{\mathrm{XpyMe}} \mathrm{TpK}$ as a glassy solid in the Schlenk tube. This glass was removed, ground to powders with a mortar and pestle, and then washed over a fine glass frit with several equivalents of pentane. The resulting free-flow powder is sufficiently pure for metalation at this step. Further purification may be performed via crystallization in a supersaturated solution in DCM layered with a few drops of MeCN left to stand overnight at $-45{ }^{\circ} \mathrm{C}$. This resulted in crystals of $\mathbf{4 a}$ and $\mathbf{4 c}$ suitable for $\mathrm{X}$-ray. NMR characterization was performed on the $\mathrm{MeCN}$ adducts of ligands $4 \mathbf{a}-\mathbf{4 e}$. Reported yields, however, correspond to ${ }^{\mathrm{XpyM}} \mathrm{TpK} \mathbf{4 a}-\mathbf{4 e}$ with coordinated MeCN removed in vacuo.

${ }^{\text {CF3pyMe }}{ }^{2 p K}$ (4a). 3a (1.626 g, $7.16 \mathrm{mmol}$ ) was reacted with ${ }^{\mathrm{iPr} 2} \mathrm{TpK}$ (3.981 g, $7.89 \mathrm{mmol}, 1.1$ equiv.), yielding $4 \mathrm{a}$ as a white powder $(1.919 \mathrm{~g}, 3.31 \mathrm{mmol}, 46 \%) .{ }^{1} \mathrm{H} \mathrm{NMR}\left(\mathrm{CDCl}_{3}\right): \delta_{\mathrm{H}} 8.58(\mathrm{bs}, 1 \mathrm{H}), 7.82(\mathrm{dd}$, $J=8.5,2.0 \mathrm{~Hz}, 1 \mathrm{H}), 7.53(\mathrm{~d}, J=8.5 \mathrm{~Hz}, 1 \mathrm{H}), 6.30$ (s, $1 \mathrm{H}), 5.79$ (s, $2 \mathrm{H}), 3.10$ (hept, $J=6.9 \mathrm{~Hz}, 2 \mathrm{H})$, 2.71 (hept, $J=6.9 \mathrm{~Hz}, 2 \mathrm{H}), 2.17$ (s, $3 \mathrm{H}), 1.97$ (s, $(\mathrm{MeCN}), 3 \mathrm{H}), 1.14$ (d, $J=6.9 \mathrm{~Hz}, 6 \mathrm{H}), 1.06$ (d, $J=$ $6.9 \mathrm{~Hz}, 6 \mathrm{H}) 1.02(\mathrm{~d}, J=6.9 \mathrm{~Hz}, 6 \mathrm{H}), 1.00(\mathrm{~d}, J=6.9 \mathrm{~Hz}, 6 \mathrm{H}) .{ }^{13} \mathrm{C} \mathrm{NMR}\left(\mathrm{CDCl}_{3}\right): \delta_{\mathrm{C}} 158.3,157.2$, 156.2, $148.0146 .3,145.6,133.7,124.0$ (q, $J=271.8 \mathrm{~Hz}), 123.5$ (q, $J=33 \mathrm{~Hz}), 119.9,116.6,104.3,96.9$, 28.0, 26.2, 24.0, 23.5, 23.4, 23.2, 22.7, 12.4, 2.0. ${ }^{9} \mathrm{~F} \mathrm{NMR}\left(\mathrm{CDCl}_{3}\right): \delta_{\mathrm{F}}=-62.3$. IR: $2453 \mathrm{~cm}^{-1}\left(v_{\mathrm{BH}}\right)$. Anal. Calcd. for $\mathrm{C}_{28} \mathrm{H}_{38} \mathrm{BF}_{3} \mathrm{KN}_{7}$ (4a): C, 58.03; H, 6.61; N, 16.92. Found: C, 57.74; H, 6.78; N, 16.74 .

${ }^{\text {pymMe }}$ TpK (4b). 3b (0.580 g, $\left.3.62 \mathrm{mmol}\right)$ was reacted with ${ }^{\mathrm{iPr} 2} \mathrm{TpK}(2.024 \mathrm{~g}, 4.01 \mathrm{mmol})$ yielding $4 \mathbf{b}$ as an off-white powder $(0.871 \mathrm{~g}, 1.70 \mathrm{mmol}, 47 \%) .{ }^{1} \mathrm{H} \mathrm{NMR}\left(\mathrm{MeCN}-d_{3}\right): \delta_{\mathrm{H}} 8.69(\mathrm{~d}, J=4.9 \mathrm{~Hz}, 2 \mathrm{H}), 7.16(\mathrm{t}$, $J=4.9 \mathrm{~Hz}, 1 \mathrm{H}), 6.51(\mathrm{~s}, 1 \mathrm{H}), 5.74$ (s, $2 \mathrm{H}), 3.40$ (hept, $J=6.9 \mathrm{~Hz}, 2 \mathrm{H}), 2.89$ (hept, $J=6.9 \mathrm{~Hz}, 2 \mathrm{H}$ ), 2.37 (s, $3 \mathrm{H}), 1.14(\mathrm{~d}, J=6.9 \mathrm{~Hz}, 6 \mathrm{H}), 1.12(\mathrm{~d}, J=6.9 \mathrm{~Hz}, 6 \mathrm{H}), 1.08(\mathrm{~d}, J=6.9 \mathrm{~Hz}, 6 \mathrm{H}), 1.07(\mathrm{~d}, J=$ $6.9 \mathrm{~Hz}, 6 \mathrm{H}) .{ }^{13} \mathrm{C} \mathrm{NMR}\left(\mathrm{MeCN}_{-} \mathrm{d}_{3}\right): \delta_{\mathrm{C}} 163.05,158.77,158.33,155.87,144.90,119.47,105.71,30.91$, 
28.95, 27.11, 24.19, 23.71, 13.29, 1.77. IR: $2446 \mathrm{~cm}^{-1}\left(v_{\mathrm{BH}}\right)$. ESI-HRMS (4b): m/z 513.3017; Calc. for $\left[{ }^{\mathrm{pymMe}} \mathrm{TpKH}\right]^{+}: 513.3022$.

${ }^{p y M e} \operatorname{TpK}$ (4c). 3c (0.818 g, $\left.5.14 \mathrm{mmol}\right)$ mixed with ${ }^{\mathrm{iPr} 2} \mathrm{TpK}$ (2.832 g, $5.61 \mathrm{mmol}, 1.1$ equiv.) yielding $4 \mathbf{c}$ as a white powder $(1.438 \mathrm{~g}, 2.81 \mathrm{mmol}, 55 \%) .{ }^{1} \mathrm{H} \mathrm{NMR}\left(\mathrm{CDCl}_{3}\right): \delta_{\mathrm{H}} 8.29(\mathrm{~d}, J=4.8 \mathrm{~Hz}, 1 \mathrm{H}), 7.60(\mathrm{ddd}, J$ $=8.3,7.7,1.8 \mathrm{~Hz}, 1 \mathrm{H}), 7.49(\mathrm{~d}, J=7.7 \mathrm{~Hz}, 1 \mathrm{H}), 7.02(\mathrm{ddd}, J=8.3,4.8,1.0,1 \mathrm{H}), 6.30(\mathrm{~s}, 1 \mathrm{H}), 5.77(\mathrm{~s}$, $2 \mathrm{H}$ ), 3.20 (hept, $J=6.8 \mathrm{~Hz}, 2 \mathrm{H}), 2.71$ (hept, $J=6.9 \mathrm{~Hz}, 2 \mathrm{H}), 2.23$ (s, $3 \mathrm{H}), 1.97$ (s, $6 \mathrm{H}(2, \mathrm{MeCN})$ ), $1.15(\mathrm{~d}, J=6.9 \mathrm{~Hz}, 6 \mathrm{H}), 1.09$ (d, $J=6.9 \mathrm{~Hz}, 6 \mathrm{H}), 1.04(\mathrm{~d}, J=6.8 \mathrm{~Hz}, 6 \mathrm{H}), 1.02(J=\mathrm{d}, 6.8 \mathrm{~Hz}, 6 \mathrm{H})$. ${ }^{13} \mathrm{C} \mathrm{NMR}\left(\mathrm{CDCl}_{3}\right): \delta_{\mathrm{C}} 157.8,155.8,154.4,149.2,144.7,136.6,121.0,120.7,96.6,28.0,26.3,23.9,23.6$, 23.4, 23.3, 12.6, 2.0. IR, $2464 \mathrm{~cm}^{-1}\left(v_{\mathrm{BH}}\right)$. ESI-HRMS (4c): $\mathrm{m} / \mathrm{z}$ 512.3064; Calc. for $\left[{ }^{\text {pyMe }} \mathrm{TpKH}\right]^{+}$: 513.3070 .

${ }^{D M A P y M e}$ TpK (4e). 3c (0.893 g, $\left.4.42 \mathrm{mmol}\right)$ mixed with ${ }^{\mathrm{iPr} 2} \mathrm{TpK}(2.820 \mathrm{~g}, 5.59 \mathrm{mmol}, 1.3$ equiv.) and 3e yielding 4c (1.468 g, $2.65 \mathrm{mmol}, 60 \%) .{ }^{1} \mathrm{H}$ NMR $\left(\mathrm{MeCN}-d_{3}\right): \delta_{\mathrm{H}}=8.16(\mathrm{~d}, J=5.9 \mathrm{~Hz}, 1 \mathrm{H}), 6.82(\mathrm{~d}, J=$ $2.6 \mathrm{~Hz}, 1 \mathrm{H}), 6.46(\mathrm{dd}, J=5.9,2.6 \mathrm{~Hz}, 1 \mathrm{H}), 6.32$ (s, $1 \mathrm{H}), 5.76$ (s, $2 \mathrm{H}), 5.45$ (s, $1 \mathrm{H}), 3.39$ (hept., $J=7.0$ Hz, 2 H), 2.99 (s, 6 H), 2.89 (hept., $J=7.0$ Hz, 2 H), 2.34 (s, 3 H), 1.15 (d, $J=7.0 \mathrm{~Hz}, 6$ H), 1.13 (d, $J=$ $7.0 \mathrm{~Hz}, 6 \mathrm{H}), 1.09$ (d, $J=7.0 \mathrm{~Hz}, 12 \mathrm{H}) .{ }^{13} \mathrm{C} \mathrm{NMR}\left(\mathrm{MeCN}-d_{3}\right): \delta_{\mathrm{C}}=158.51,156.18,155.76,154.82$, 151.04, 150.59, 144.29, 105.73, 103.62, 102.99, 96.67, 28.94, 27.10, 24.20, 24.15, 23.72, 13.21. IR: 2449 $\mathrm{cm}^{-1}\left(v_{\mathrm{BH}}\right)$. ESI-HRMS (4e): m/z 555.3489; Calc. for [ $\left.{ }^{\text {DMAPMe }} \mathrm{TpKH}\right]^{+}: 555.3492$.

\section{General Synthesis of ${ }^{\mathrm{XpyMe}} \mathrm{TpCuOAc}$ Complexes 5a - 5e}

In a $22 \mathrm{~mL}$ scintillation vial, the naked ${ }^{\mathrm{XpyMe}} \mathrm{TpK}$ ligand was dissolved in $5 \mathrm{~mL}$ of DCM and mixed with a suspension of anhydrous $\mathrm{Cu}(\mathrm{OAc})_{2}$ in $5 \mathrm{~mL}$ of $\mathrm{DCM}$. The mixture was allowed to stir for $14 \mathrm{~h}$ and then was filtered through a Celite pad to give a light blue filtrate, which was then concentrated to dryness and washed with $3 \times 5 \mathrm{~mL}$ portions of $\mathrm{MeCN}$. A light blue powder was then recrystallized out of a supersaturated DCM solution left to stand at $-45^{\circ} \mathrm{C}$ overnight.

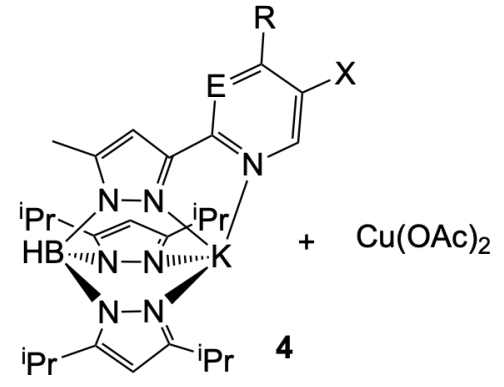
(4a) $\mathrm{E}=\mathrm{CH}, \mathrm{R}=\mathrm{H}, \mathrm{X}=\mathrm{CF}_{3}$
(4b) $E=N, R=H, X=H$
(4c) $\mathrm{E}=\mathrm{CH}, \mathrm{R}=\mathrm{H}, \mathrm{X}=\mathrm{H}$
(4e) $\mathrm{E}=\mathrm{CH}, \mathrm{R}=\mathrm{NMe}_{2}, \mathrm{X}=\mathrm{H}$

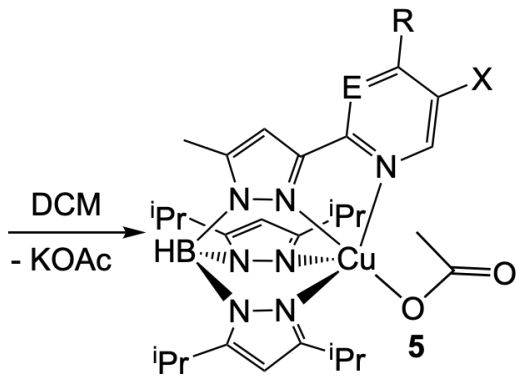

(5a) $\mathrm{E}=\mathrm{CH}, \mathrm{R}=\mathrm{H}, \mathrm{X}=\mathrm{CF}_{3}$

(5b) $\mathrm{E}=\mathrm{N}, \mathrm{R}=\mathrm{H}, \mathrm{X}=\mathrm{H}$

(5c) $\mathrm{E}=\mathrm{CH}, \mathrm{R}=\mathrm{H}, \mathrm{X}=\mathrm{H}$

(5e) $\mathrm{E}=\mathrm{CH}, \mathrm{R}=\mathrm{NMe}_{2}, \mathrm{X}=\mathrm{H}$

Scheme S6. The general synthesis of ${ }^{\mathrm{XpyMe}} \mathrm{TpK}$ ligands $4 \mathbf{a}-\mathbf{4 e}$. 
${ }^{C F 3 p y M e}$ TpCuOAc (5a). 4a (0.926 g, $\left.1.60 \mathrm{mmol}\right)$ reacted with $\mathrm{Cu}(\mathrm{OAc}){ }_{2}(0.296 \mathrm{~g}, 1.63 \mathrm{mmol})$ in a $100 \mathrm{~mL}$ round-bottom flask charged with $50 \mathrm{~mL}$ of DCM and a stir bar. The reaction yielded 5a $(0.761 \mathrm{~g}, 1.15$ mmol, 72\%). UV-vis $\left(\mathrm{CH}_{2} \mathrm{Cl}_{2}, 25^{\circ} \mathrm{C}\right): \lambda_{\max }\left(\varepsilon \mathrm{M}^{-1} \mathrm{~cm}^{-1}\right)=760 \mathrm{~nm}(101) . \mu_{\mathrm{eff}}=1.9$ B.M. IR: $2531 \mathrm{~cm}^{-1}$ $\left(v_{\mathrm{BH}}\right)$. Anal. Calcd. for $\mathrm{C}_{30} \mathrm{H}_{41} \mathrm{BCuF}_{3} \mathrm{~N}_{7} \mathrm{O}_{2}(\mathbf{5 a}): \mathrm{C}, 54.34 ; \mathrm{H}, 6.23 ; \mathrm{N}, 14.79$. Found: $\mathrm{C}, 54.46 ; \mathrm{H}, 6.45 ; \mathrm{N}$, 14.68 .

A

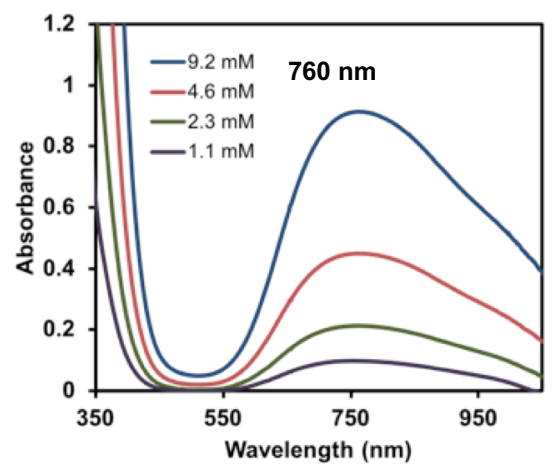

B

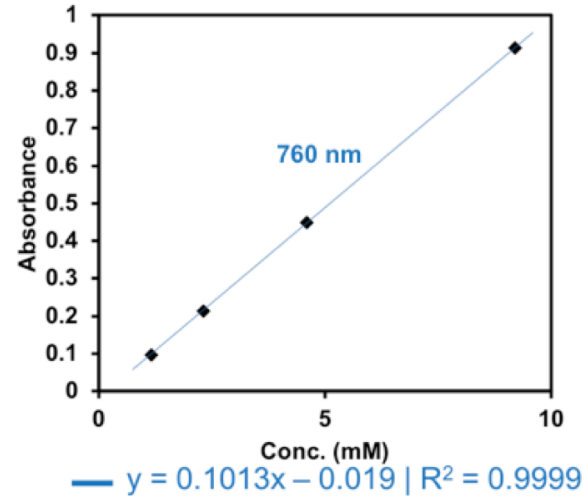

Figure S2. (A) UV-vis of 5a in DCM at $25^{\circ} \mathrm{C}$ at different concentrations $(9.2 \mathrm{mM}, 4.6 \mathrm{mM}, 2.3 \mathrm{mM}$, and $1.1 \mathrm{mM}$ ) (B) Corresponding Beer's law plot of 5 a to give $\lambda_{\max }=760 \mathrm{~nm}, \varepsilon=101 \mathrm{M}^{-1} \mathrm{~cm}^{-1}$.

${ }^{\text {pymMe }} \mathrm{TpCuOAc}(\mathbf{5 b}) .4 \mathbf{b}(0.160 \mathrm{~g}, 0.312 \mathrm{mmol})$ reacted with $\mathrm{Cu}(\mathrm{OAc})_{2}(0.058 \mathrm{~g}, 0.320 \mathrm{mmol})$ yielded $\mathbf{5 b}$ $(0.146 \mathrm{~g}, 0.245 \mathrm{mmol}, 78 \%)$. UV-vis $\left(\mathrm{CH}_{2} \mathrm{Cl}_{2}, 25^{\circ} \mathrm{C}\right): \lambda\left(\varepsilon \mathrm{M}^{-1} \mathrm{~cm}^{-1}\right)=700 \mathrm{~nm}(105), 814 \mathrm{~nm}(126) . \mu_{\mathrm{eff}}=$ 1.9 B.M. IR: $2538 \mathrm{~cm}^{-1}\left(v_{\mathrm{BH}}\right)$. Anal. Calcd. for $\mathrm{C}_{28} \mathrm{H}_{41} \mathrm{BCuN}_{8} \mathrm{O}_{2}(\mathbf{5 b})$ : C, 56.42; H, 6.93; N, 18.80. Found: C, 56.50; H, 7.12; N, 18.41 .

A

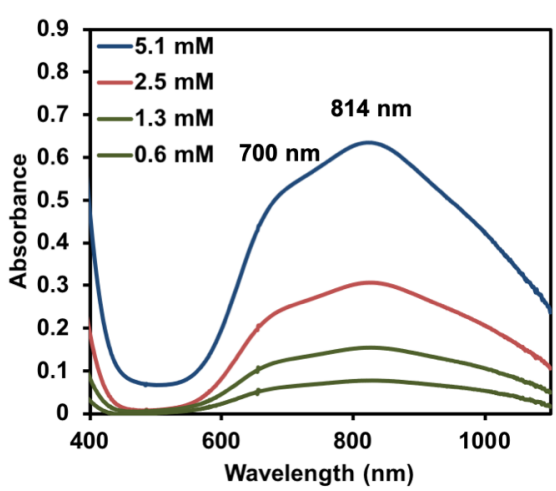

B

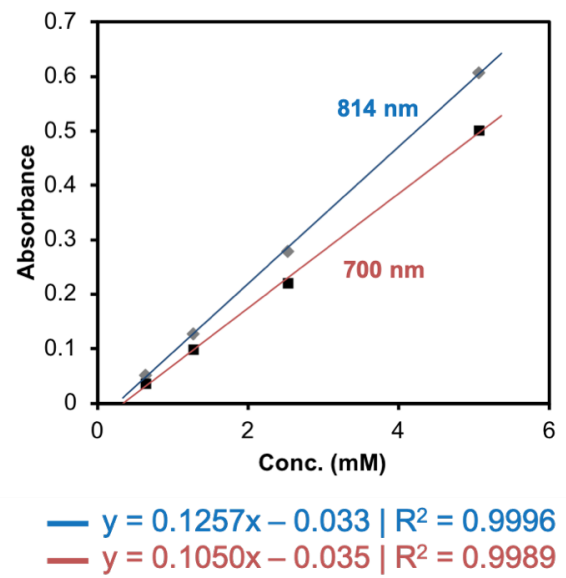

Figure S3. (A) UV-vis of $\mathbf{5 b}$ in DCM at $25^{\circ} \mathrm{C}$ at different concentrations $(5.1 \mathrm{mM}, 2.5 \mathrm{mM}, 1.3 \mathrm{mM}$, and $0.6 \mathrm{mM}$ ) (B) Corresponding Beer's law plot of $\mathbf{5 b}$ to give $\lambda_{\max }=814 \mathrm{~nm}, \varepsilon=126 \mathrm{M}^{-1} \mathrm{~cm}^{-1} ; \lambda=700(\mathrm{sh})$, $\varepsilon=105 \mathrm{M}^{-1} \mathrm{~cm}^{-1}$. 
${ }^{p y M e}$ TpCuOAc (5c). 4c $(0.482 \mathrm{~g}, 0.941 \mathrm{mmol})$ reacted with $\mathrm{Cu}(\mathrm{OAc})_{2}(0.171 \mathrm{~g}, 0.943 \mathrm{mmol})$ yielded $\mathbf{5 c}$ (0.493 g, $0.823 \mathrm{mmol}, 88 \%$ yield). UV-vis $\left(\mathrm{CH}_{2} \mathrm{Cl}_{2}, 25{ }^{\circ} \mathrm{C}\right): \lambda\left(\varepsilon \mathrm{M}^{-1} \mathrm{~cm}^{-1}\right)=670 \mathrm{~nm}(82), 820 \mathrm{~nm}(113)$. $\mu_{\text {eff }}=1.9$ B.M. IR: $2535 \mathrm{~cm}^{-1}\left(v_{\mathrm{BH}}\right)$. Anal. Calcd for $\mathrm{C}_{29} \mathrm{H}_{42} \mathrm{BCuN}_{7} \mathrm{O}_{2}(\mathbf{5 c}) \mathrm{C}, 58.54 ; \mathrm{H}, 7.11 ; \mathrm{N}, 16.48$. Found: C, 58.65; H, 7.36; N, 16.35 .

A

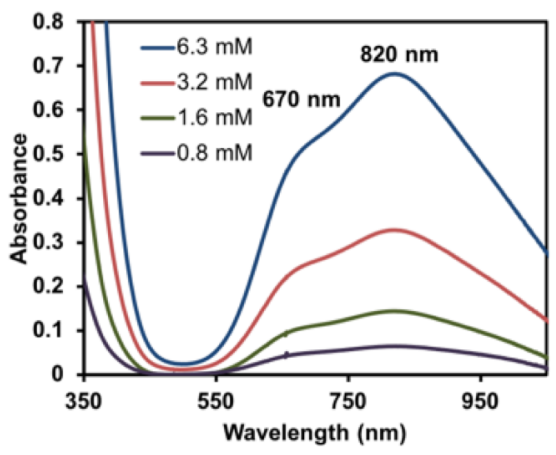

B

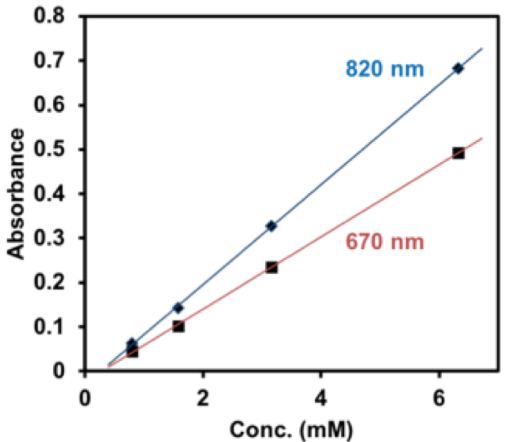

$-y=0.1127 x-0.030 \mid R^{2}=0.9998$

$-y=0.0816 x-0.023 \mid R^{2}=0.9997$

Figure S4. (A) UV-vis of $\mathbf{5 c}$ in DCM at $25^{\circ} \mathrm{C}$ at different concentrations $(6.3 \mathrm{mM}, 3.2 \mathrm{mM}, 1.6 \mathrm{mM}$, and $0.8 \mathrm{mM}$ ) (B) Corresponding Beer's law plot of $5 \mathbf{c}$ to give $\lambda_{\max }=820 \mathrm{~nm}, \varepsilon=113 \mathrm{M}^{-1} \mathrm{~cm}^{-1} ; \lambda=670(\mathrm{sh})$, $\varepsilon=82 \mathrm{M}^{-1} \mathrm{~cm}^{-1}$.

${ }^{D M A P M e}$ TpCuOAc (5e). 4e (0.578 g, $\left.1.04 \mathrm{mmol}\right)$ reacted with $\mathrm{Cu}(\mathrm{OAc})_{2}(0.196 \mathrm{~g}, 1.08 \mathrm{mmol})$ yielded 5e ( $0.523 \mathrm{~g}, 0.820 \mathrm{mmol}, 79 \%)$. UV-vis $\left(\mathrm{CH}_{2} \mathrm{Cl}_{2}, 25^{\circ} \mathrm{C}\right): \lambda_{\max }\left(\varepsilon \mathrm{M}^{-1} \mathrm{~cm}^{-1}\right)=688 \mathrm{~nm}$ (99) $798 \mathrm{~nm}\left(134 \mathrm{M}^{-}\right.$ $\left.{ }^{1} \mathrm{~cm}^{-1}\right) . \mu_{\text {eff }}=1.9$ B.M. IR: $2531 \mathrm{~cm}^{-1}\left(v_{\mathrm{BH}}\right)$. Anal. Calcd. for $\mathrm{C}_{31} \mathrm{H}_{47} \mathrm{BCuN}_{8} \mathrm{O}_{2}(\mathbf{5 e}): \mathrm{C}, 58.35 ; \mathrm{H}, 7.42 ; \mathrm{N}$, 17.56. Found: C, 58.36; H, 7.75; N, 17.42.

A

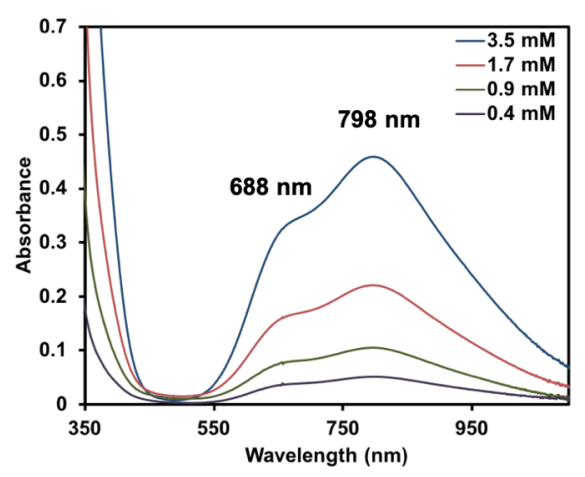

B

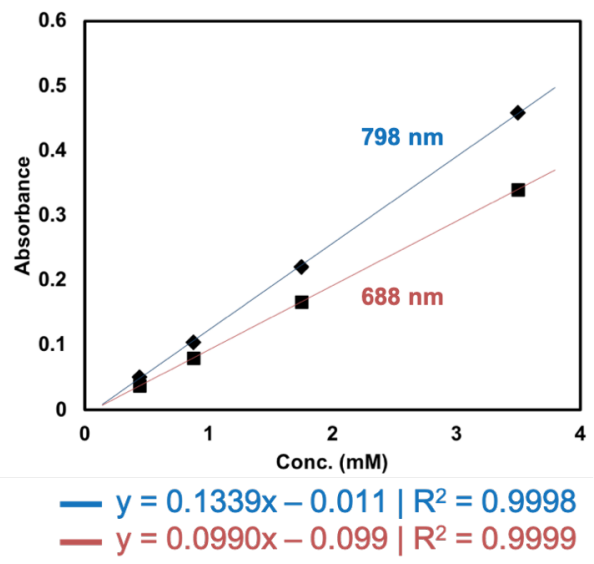

Figure S5. (A) UV-vis of 5e in DCM at $25^{\circ} \mathrm{C}$ at different concentrations $(3.5 \mathrm{mM}, 1.7 \mathrm{mM}, 0.9 \mathrm{mM}$, and $0.4 \mathrm{mM}$ ) (B) Corresponding Beer's law plot of $5 \mathbf{e}$ to give $\lambda_{\max }=798 \mathrm{~nm}, \varepsilon=134 \mathrm{M}^{-1} \mathrm{~cm}^{-1} ; \lambda=688(\mathrm{sh}), \varepsilon$ $=99 \mathrm{M}^{-1} \mathrm{~cm}^{-1}$. 


\section{General Synthesis of $\left[{ }^{\mathrm{XpyMe}} \mathrm{TpCu}\right]_{2}(\mu-\mathrm{OH})_{2}$ Complexes $6 \mathrm{a}-6 \mathrm{e}$}

Methods were adapted from Kitajima and Fujisawa's preparation of $\left[{ }^{\mathrm{iPr} 2} \mathrm{TpCu}\right]_{2}(\mu-\mathrm{OH})_{2} .{ }^{3} \mathrm{~A} 40 \mathrm{~mL} \mathrm{DCM}$ solution of ${ }^{\mathrm{XpyMe}} \mathrm{TpCuOAc}$ was prepared in a Schlenk flask charged with a magnetic stir bar. This solution was then allowed to stir under nitrogen with $10 \mathrm{~mL}$ of a nitrogen-sparged $1 \mathrm{M} \mathrm{NaOH}$ (aq) solution for 2 h. The DCM layer was then decanted and concentrated to dryness to a deep blue powder. This powder was transferred to an $\mathrm{N}_{2}$ filled glovebox, washed with $3 \times 5 \mathrm{~mL}$ portions of dry MeCN and then dissolved in DCM. The solutions were then filtered through a syringe filter, concentrated in vacuo, layered with $\mathrm{MeCN}$, and left to stand at $-45^{\circ} \mathrm{C}$ overnight to crystallize.

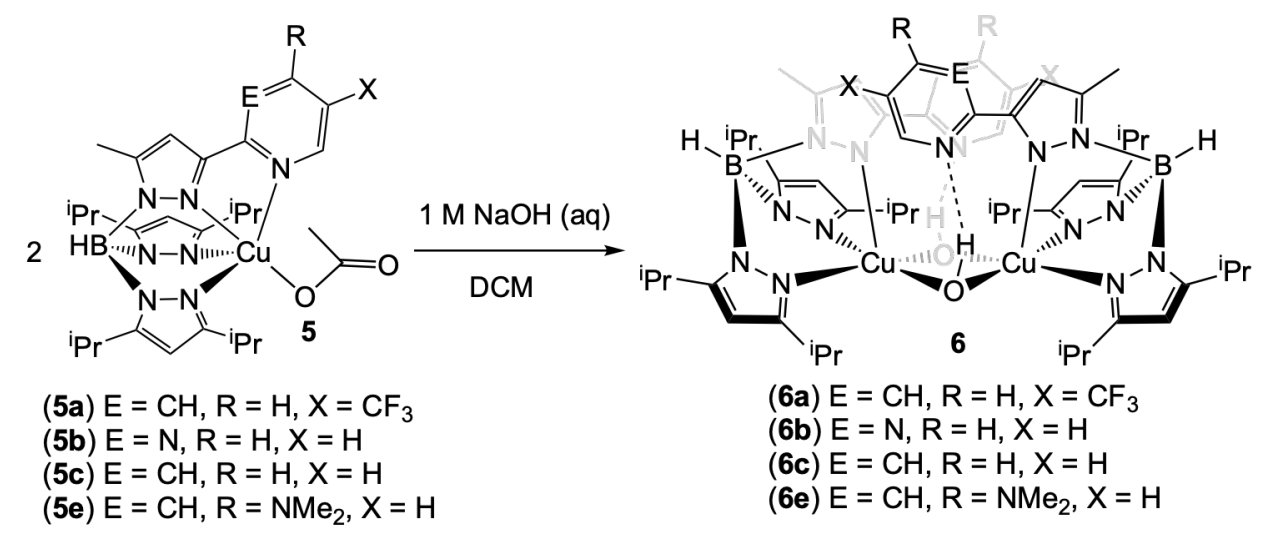

Scheme S7. The general synthesis of $\left[{ }^{\mathrm{XpyMe}} \mathrm{TpCu}\right]_{2}(\mu-\mathrm{OH})_{2}$ complexes $\mathbf{6 a}-\mathbf{6 e}$.

$\left[^{C F 3 p y M e} \mathrm{TpCu}\right]_{2}(\mu-\mathrm{OH})_{2}(\mathbf{6 a}) .5 \mathbf{5}(0.269 \mathrm{~g}, 0.406 \mathrm{mmol})$ afforded $\mathbf{6 a}$ as navy blue crystals $(0.210 \mathrm{~g}, 0.169$ mmol, 83\%). UV-vis $\left(\mathrm{CH}_{2} \mathrm{Cl}_{2}, 25^{\circ} \mathrm{C}\right): \lambda_{\max }\left(\varepsilon \mathrm{M}^{-1} \mathrm{~cm}^{-1}\right)=605 \mathrm{~nm}(103) . \mu_{\text {eff }}=2.6$ B.M. IR (KBr pellet): $3356 \mathrm{~cm}^{-1}$ (br, $v_{\mathrm{OH}}$ ). Anal. Calcd. for $\mathrm{C}_{56} \mathrm{H}_{78} \mathrm{~B}_{2} \mathrm{Cu}_{2} \mathrm{~F}_{6} \mathrm{~N}_{14} \mathrm{O}_{2}$ (6a): C, 54.15; H, 6.33; N, 15.79. Found: $\mathrm{C}$, 54.03; H, 6.33; N 15.63. 
A

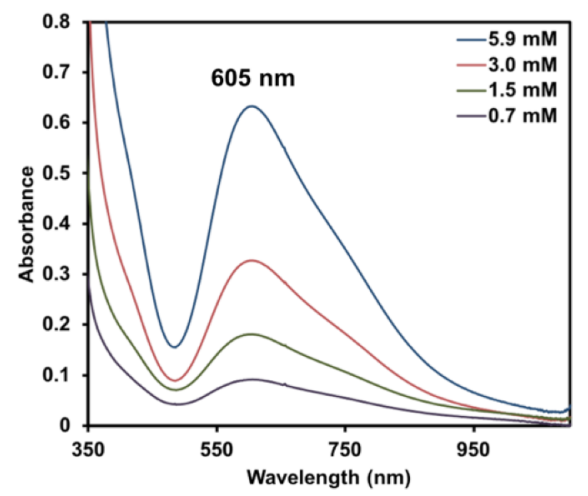

B

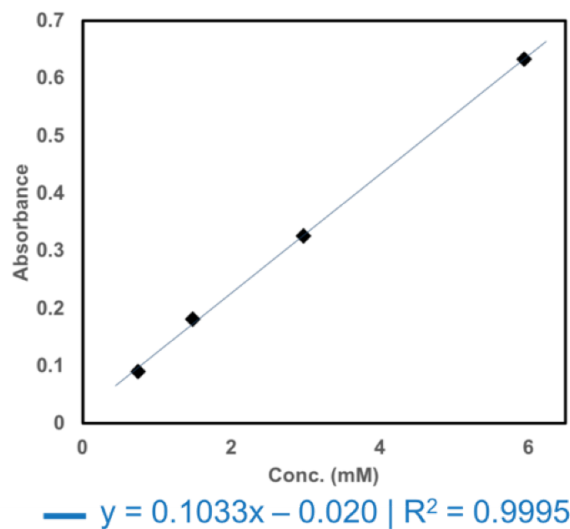

Figure S6. (A) UV-vis of 6a in DCM at $25^{\circ} \mathrm{C}$ at different concentrations $(5.9 \mathrm{mM}, 3.0 \mathrm{mM}, 1.5 \mathrm{mM}$, and $0.7 \mathrm{mM}$ ) (B) Corresponding Beer's law plot of 6a to give $\lambda_{\max }=605 \mathrm{~nm}, \varepsilon=103 \mathrm{M}^{-1} \mathrm{~cm}^{-1}$.

$\left[^{\text {pymMe }} \mathrm{TpCu}\right]_{2}(\mu-\mathrm{OH})_{2}(\mathbf{6 b}) .5 \mathbf{b}(0.141 \mathrm{~g}, 0.237 \mathrm{mmol})$ afforded $\mathbf{6 b}$ as navy blue crystals $(0.124 \mathrm{~g}, 0.112$ mmol, 95\%). UV-vis $\left(\mathrm{CH}_{2} \mathrm{Cl}_{2}, 25^{\circ} \mathrm{C}\right): \lambda\left(\varepsilon \mathrm{M}^{-1} \mathrm{~cm}^{-1}\right)=413 \mathrm{~nm}(63), 588 \mathrm{~nm}$ (100), $752 \mathrm{~nm}(49) . \mu_{\mathrm{eff}}=2.6$ B.M. IR (KBr pellet): $3525 \mathrm{~cm}^{-1}$ (br, $v_{\mathrm{OH}}$ ). Anal. Calcd. for $\mathrm{C}_{52} \mathrm{H}_{78} \mathrm{~B}_{2} \mathrm{Cu}_{2} \mathrm{~F}_{6} \mathrm{~N}_{16} \mathrm{O}_{2}(6 \mathbf{b})$ : C, 56.37, H, 7.10; N, 20.23. Found: C, 56.39; H, 7.16; N, 19.91.

A

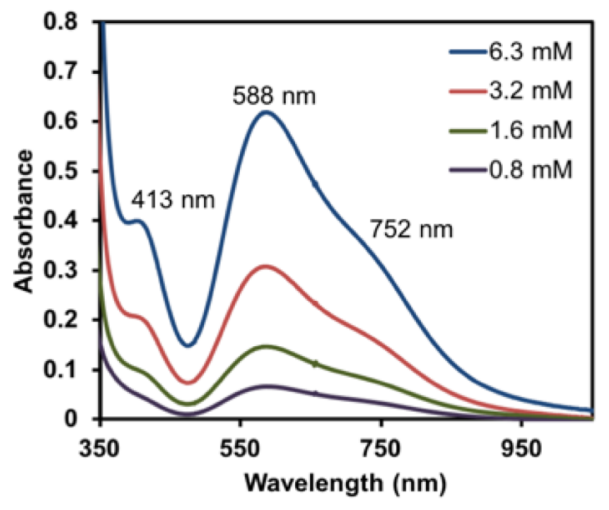

B

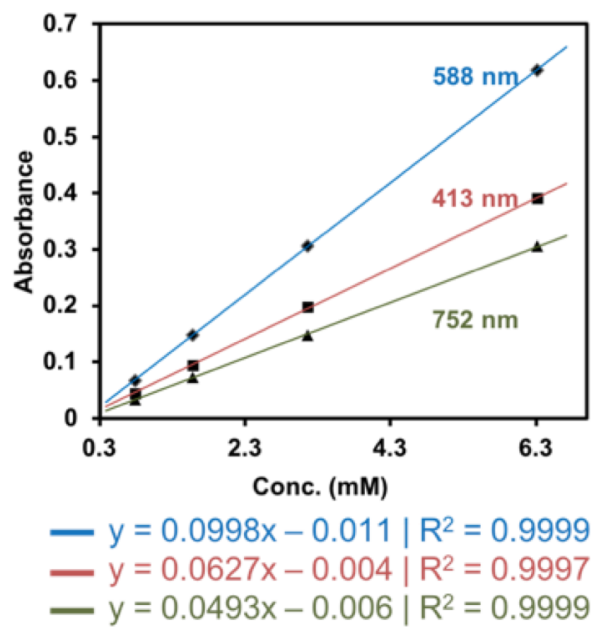

Figure S7. (A) UV-vis of $6 \mathbf{b}$ in DCM at $25^{\circ} \mathrm{C}$ at different concentrations $(6.3 \mathrm{mM}, 3.2 \mathrm{mM}, 1.6 \mathrm{mM}$, and $0.8 \mathrm{mM}$ ) (B) Corresponding Beer's law plot of $6 \mathbf{b}$ to give $\lambda_{\max }=588 \mathrm{~nm}, \varepsilon=100 \mathrm{M}^{-1} \mathrm{~cm}^{-1} ; \lambda=413 \mathrm{~nm}$, $\varepsilon=63 \mathrm{M}^{-1} \mathrm{~cm}^{-1} ; \lambda=752 \mathrm{~nm}(\mathrm{sh}), \varepsilon=49 \mathrm{M}^{-1} \mathrm{~cm}^{-1}$. 
$\left[^{p y M e} \mathrm{TpCu}\right]_{2}(\mu-\mathrm{OH})_{2}(\mathbf{6 c}) .5 \mathbf{c}(0.754 \mathrm{~g}, 1.27 \mathrm{mmol}) \mathbf{6 c}$ as cerulean crystals $(0.523 \mathrm{~g}, 0.472 \mathrm{mmol}, 75 \%)$. UV-vis $\left(\mathrm{CH}_{2} \mathrm{Cl}_{2}, 25^{\circ} \mathrm{C}\right): \lambda_{\max }\left(\varepsilon \mathrm{M}^{-1} \mathrm{~cm}^{-1}\right)=630 \mathrm{~nm}(116) . \mu_{\mathrm{eff}}=2.6$ B.M. IR (KBr pellet): $3269 \mathrm{~cm}^{-1}$ (br, $\left.v_{\mathrm{OH}}\right)$. Anal. Calcd for $\mathrm{C}_{54} \mathrm{H}_{80} \mathrm{~B}_{2} \mathrm{Cu}_{2} \mathrm{~N}_{14} \mathrm{O}_{2}(6 \mathrm{c})$ : C, 58.64; H, 7.29; N, 17.7. Found: C, 58.60; H, 7.46; N, 17.57 .

A

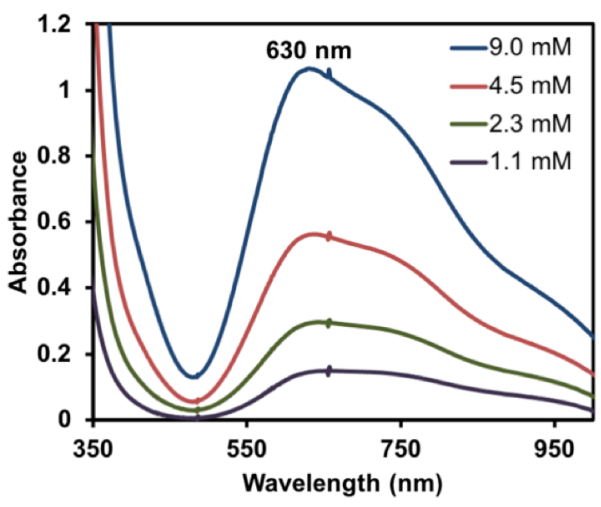

B

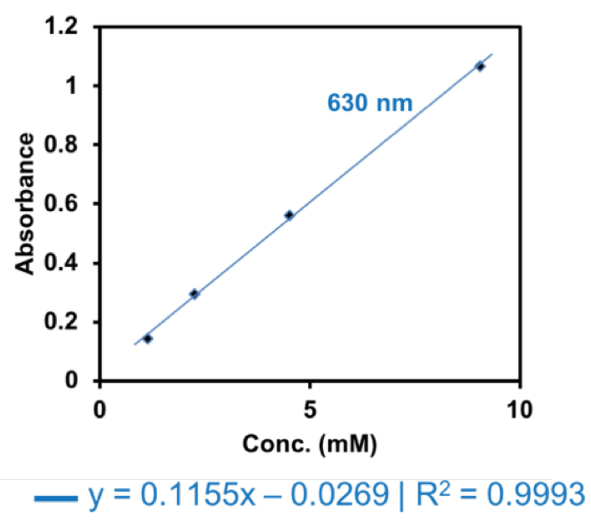

Figure S8. (A) UV-vis of $6 \mathbf{c}$ in DCM at $25^{\circ} \mathrm{C}$ at different concentrations $(6.3 \mathrm{mM}, 3.2 \mathrm{mM}, 1.6 \mathrm{mM}$, and $0.8 \mathrm{mM}$ ) (B) Corresponding Beer's law plot of $6 \mathbf{c}$ to give $\lambda_{\max }=630 \mathrm{~nm}, \varepsilon=116 \mathrm{M}^{-1} \mathrm{~cm}^{-1}$.

$\left[^{D M A P M e} T p C u(\mu-O H)_{2}(6 \mathbf{6 e}) .5 e(0.133 \mathrm{~g}, 0.209 \mathrm{mmol})\right.$ afforded $\mathbf{6 e}$ as indigo crystals $(0.104 \mathrm{~g}, 0.087 \mathrm{mmol}$, 84\%). UV-vis $\left(\mathrm{CH}_{2} \mathrm{Cl}_{2}, 25^{\circ} \mathrm{C}\right): \lambda_{\max }\left(\varepsilon \mathrm{M}^{-1} \mathrm{~cm}^{-1}\right)=644 \mathrm{~nm}$ (118). $\mu_{\mathrm{eff}}=2.7$ B.M. IR (Thin film evaporated from DCM on $\mathrm{KBr}$ window): $3217 \mathrm{~cm}^{-1}$ (br, $v_{\mathrm{OH}}$ ). Anal. Calcd. for $\mathrm{C}_{58} \mathrm{H}_{90} \mathrm{~B}_{2} \mathrm{Cu}_{2} \mathrm{~N}_{16} \mathrm{O}_{2}(\mathbf{6 e})$ : C, 58.43; H, 7.61; N, 18.80. Found: C, 58.17; H, 7.93; N, 18.50.

A

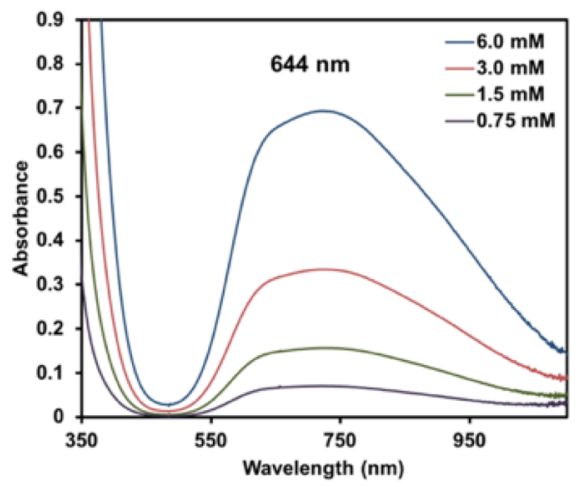

B

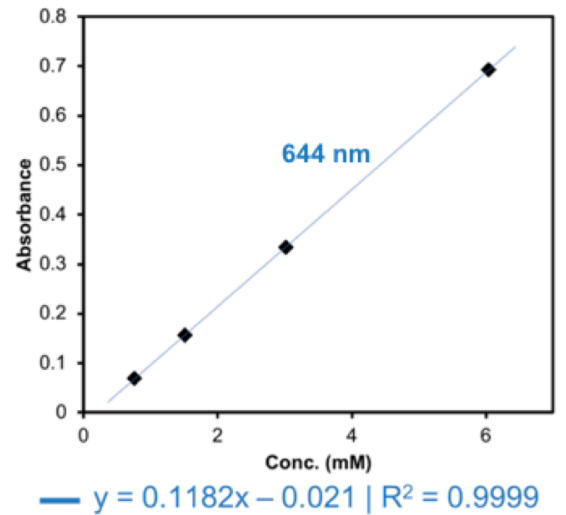

Figure S9. (A) UV-vis of $6 \mathrm{e}$ in $\mathrm{DCM}$ at $25^{\circ} \mathrm{C}$ at different concentrations $(6.0 \mathrm{mM}, 3.0 \mathrm{mM}, 1.5 \mathrm{mM}$, and 0.8 $\mathrm{mM}$ ) (B) Corresponding Beer's law plot of $6 \mathbf{e}$ to give $\lambda_{\max }=644 \mathrm{~nm}, \varepsilon=118 \mathrm{M}^{-1} \mathrm{~cm}^{-1}$. 
IR Characterization of $\left[{ }^{\mathrm{XpyMe}} \mathrm{TpCu}\right]_{2}(\mu-\mathrm{OH})_{2}$ Complexes 6a-6e
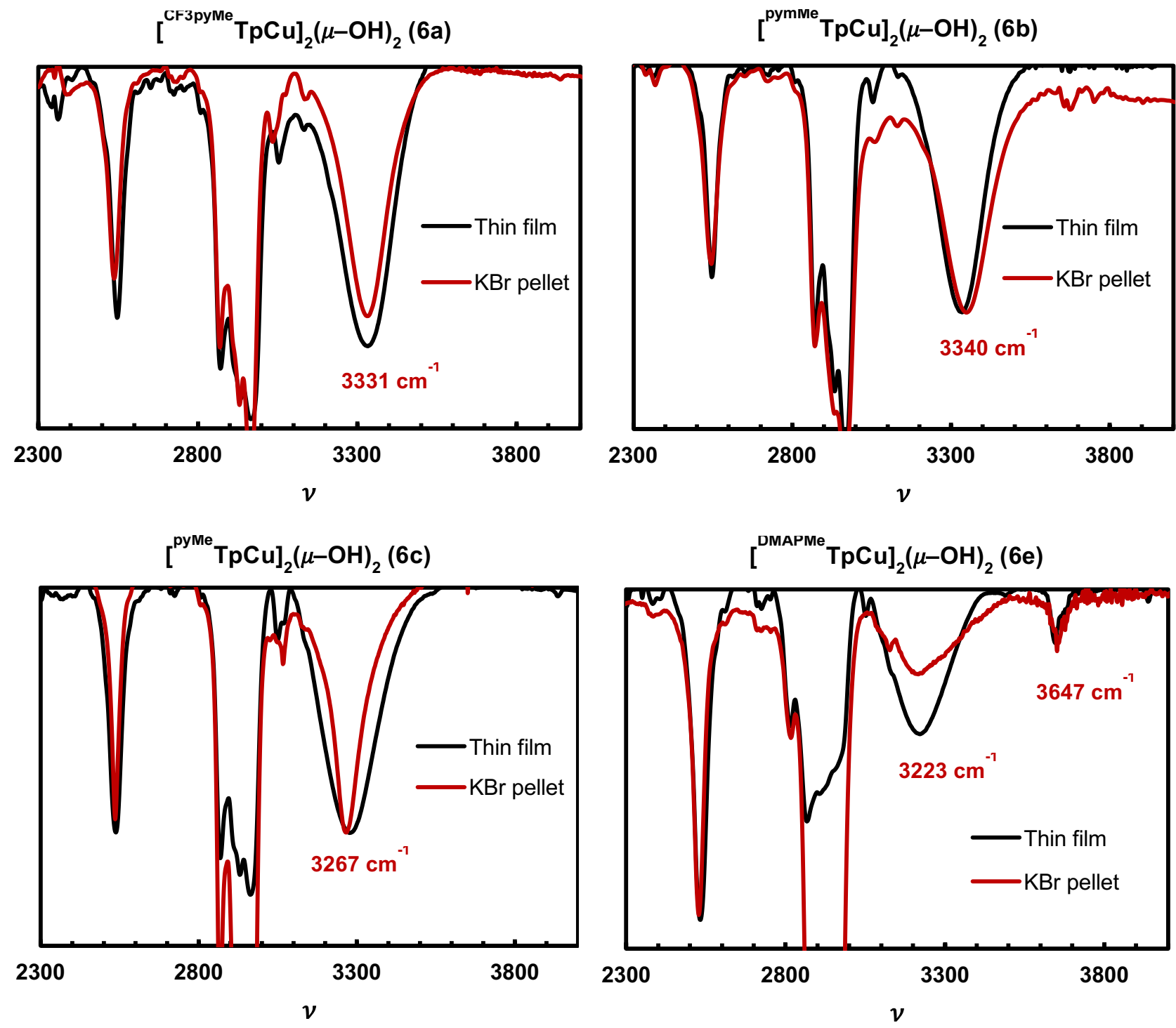

Figure S10. Overlay of IR spectra taken of each $\left[{ }^{\mathrm{XpyMe}} \mathrm{TpCu}\right]_{2}(\mu-\mathrm{OH})_{2}$ taken as a thin film evaporated from $\mathrm{DCM}$ on a $\mathrm{KBr}$ plate (black) and IR spectra of the respective compounds taken as powders in a $\mathrm{KBr}$ pellet (red). 


\section{General Procedure for Titration of $\left[{ }^{\mathrm{XpyMe}} \mathrm{TpCu}\right]_{2}(\mu-\mathrm{OH})_{2}$ Complexes 6a-6e}

Titrations of $\mathbf{6 a}-\mathbf{6 e}$ were monitored using a Varian Cary 8454 UV-vis spectrometer set to kinetic array mode with scans occurring at $20 \mathrm{~s}$ intervals. Solutions of $6 \mathbf{a}-\mathbf{6 e}$ were prepared in $2.5 \mathrm{~mL}$ of DCM and transferred to a quartz cuvette capped with a rubber septum chilled to $-10{ }^{\circ} \mathrm{C}$ unless otherwise noted. Solutions of HOTf or select bases in DCM were freshly prepared in the glovebox transferred sequentially using a Hamilton $880115 \mu \mathrm{L}$ syringe. 


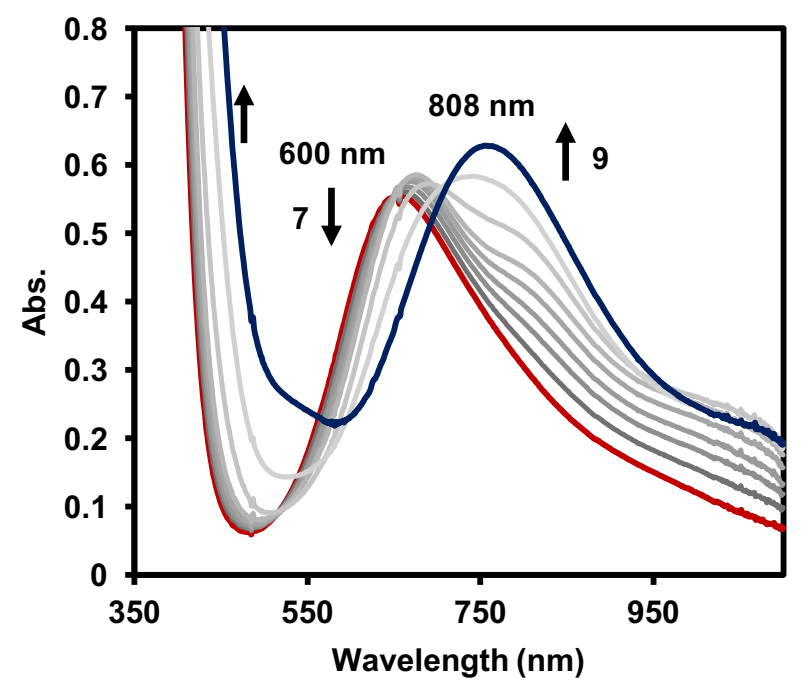

Figure S11. The titration of $\left[{ }^{1 \mathrm{Ir} /} \mathrm{TpCu}\right]_{2}(\mu-\mathrm{OH})_{2}(7)$ by HOTf at $-40{ }^{\circ} \mathrm{C}$. The red trace indicates the initial spectrum of 7, gray traces correspond to species present after each addition of titrant HOTf (ca. 0.1 equiv. per portion), and the blue trace indicates species 9 formed at the endpoint. While a transformation clearly takes place, no clean isosbestic point is observed.
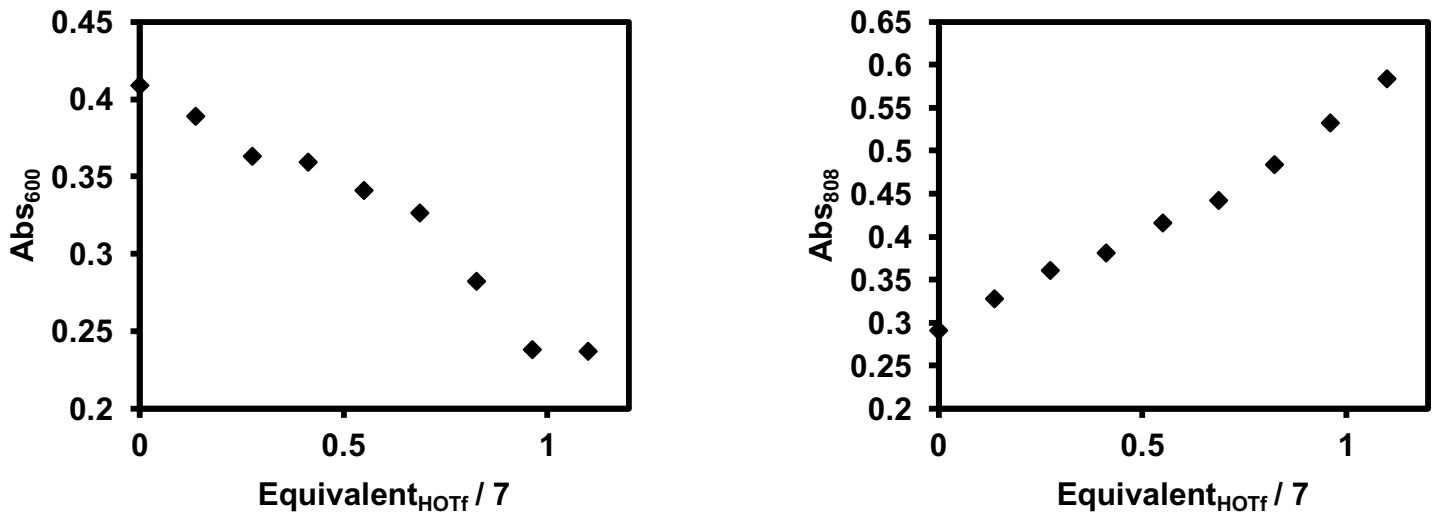

Figure S12. Monitoring the decay in the absorption at $600 \mathrm{~nm}$ (left) and growth in the absorption at 808 (right) during the addition of HOTf. After the addition of nearly 0.96 equiv. HOTf, no further change in the absorbance at $600 \mathrm{~nm}$ occurs, indicating a potential endpoint. 

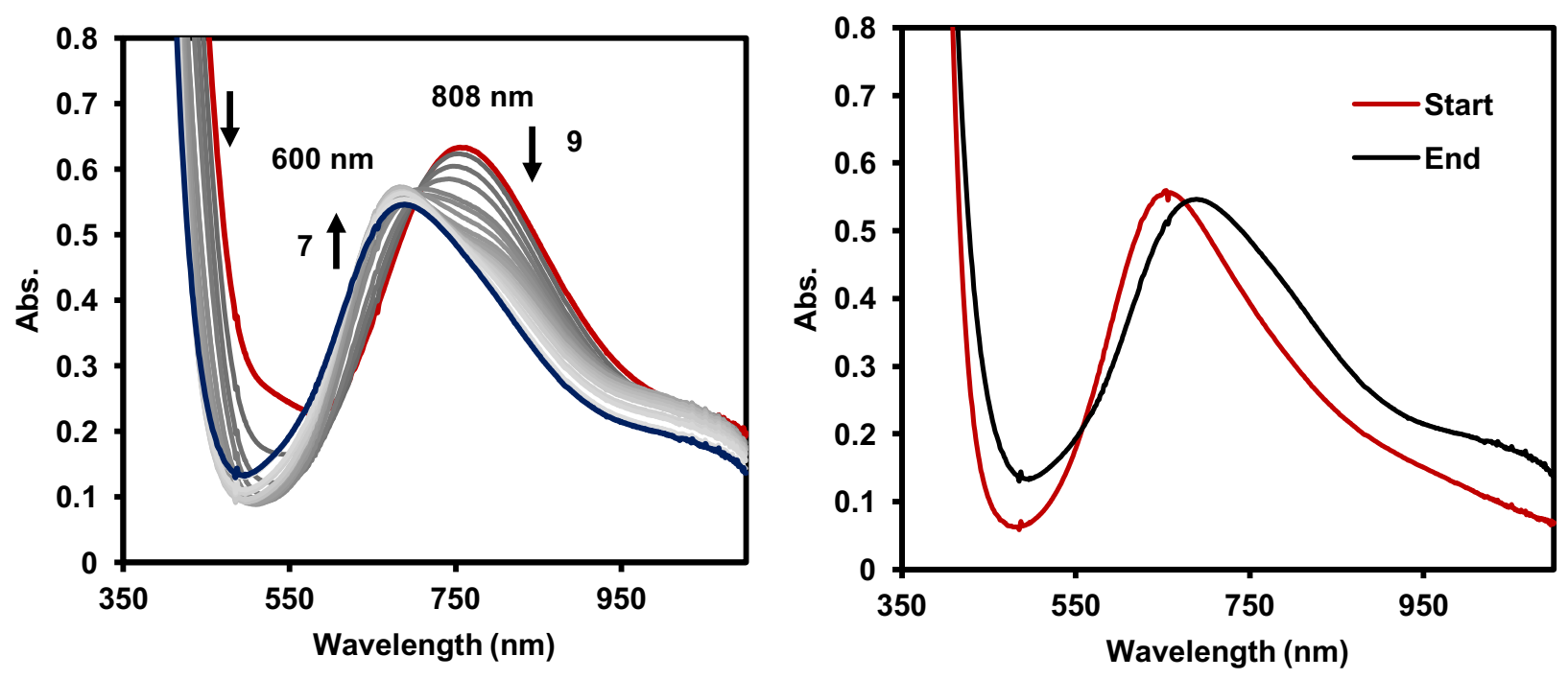

Figure S13. (Left) The backtitration of 9 (previously formed in situ by addition of 1 equiv. HOTf to 7) with collidine at $-40{ }^{\circ} \mathrm{C}$. The red trace corresponds to 9 while each gray trace corresponds to the addition of each portion of collidine (ca. 0.2 equiv). The blue trace represents the end point of the titration. Overlaying traces of 7 before (red trace, right) and after the titration/backtitration sequence indicates some irreversible change in the original compound.
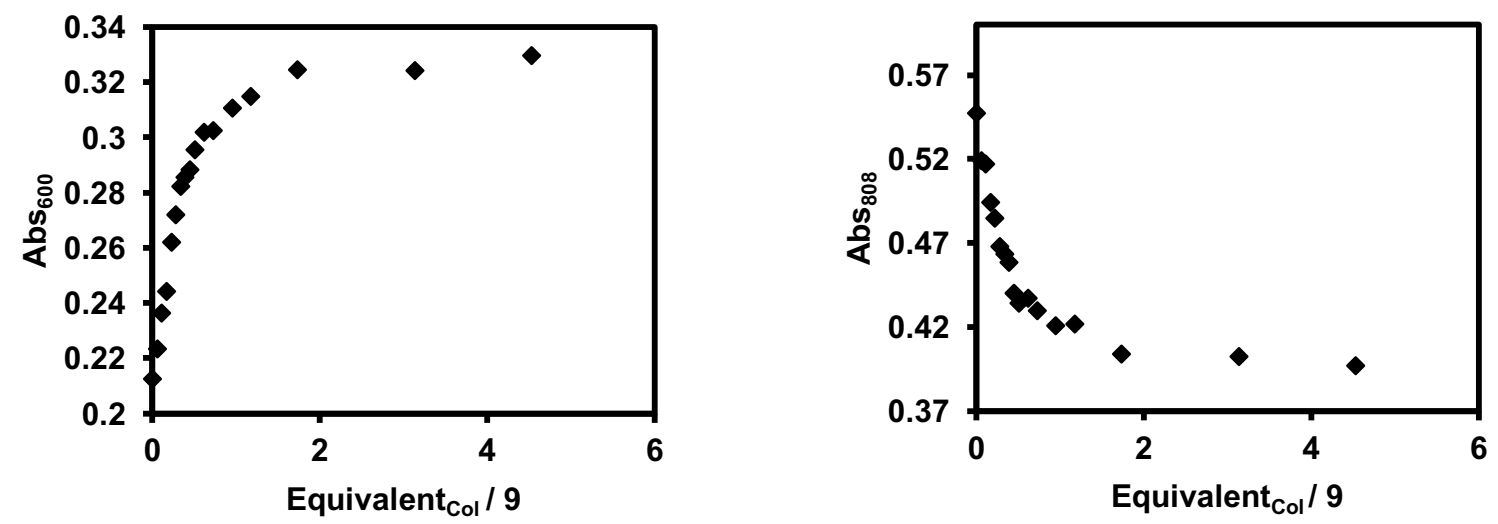

Figure S14. Added equivalents of collidine that result in the growth of the bands at $600 \mathrm{~nm}$ (left) and loss of $808 \mathrm{~nm}$ (right) that correspond to 7 and $\mathbf{9}$, respectively. Judged by the change in $600 \mathrm{~nm}$ and 808 $\mathrm{nm}$, the reaction of 9 is sufficiently complete after the addition of 2 equiv. collidine. 


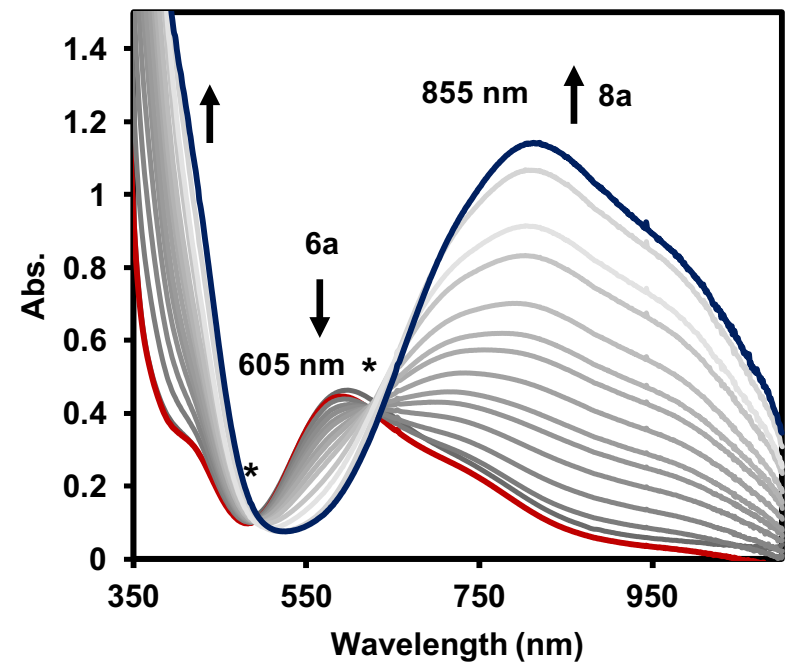

Figure S15. The titration of $\left[{ }^{\mathrm{CF} 3 \mathrm{pyMe}} \mathrm{TpCu}\right]_{2}(\mu-\mathrm{OH})_{2}(\mathbf{6 a})$ by HOTf at $-40{ }^{\circ} \mathrm{C}$. The red trace represents the initial spectrum of $\mathbf{6 a}$, gray traces correspond to each addition of titrant HOTf (ca. 0.2 equiv. each), and the blue trace correspond to $\mathbf{8 a}$ formed at the endpoint. The presence of isosbestic points indicates clean formation $8 \mathbf{a}$ from $\mathbf{6 a}$.
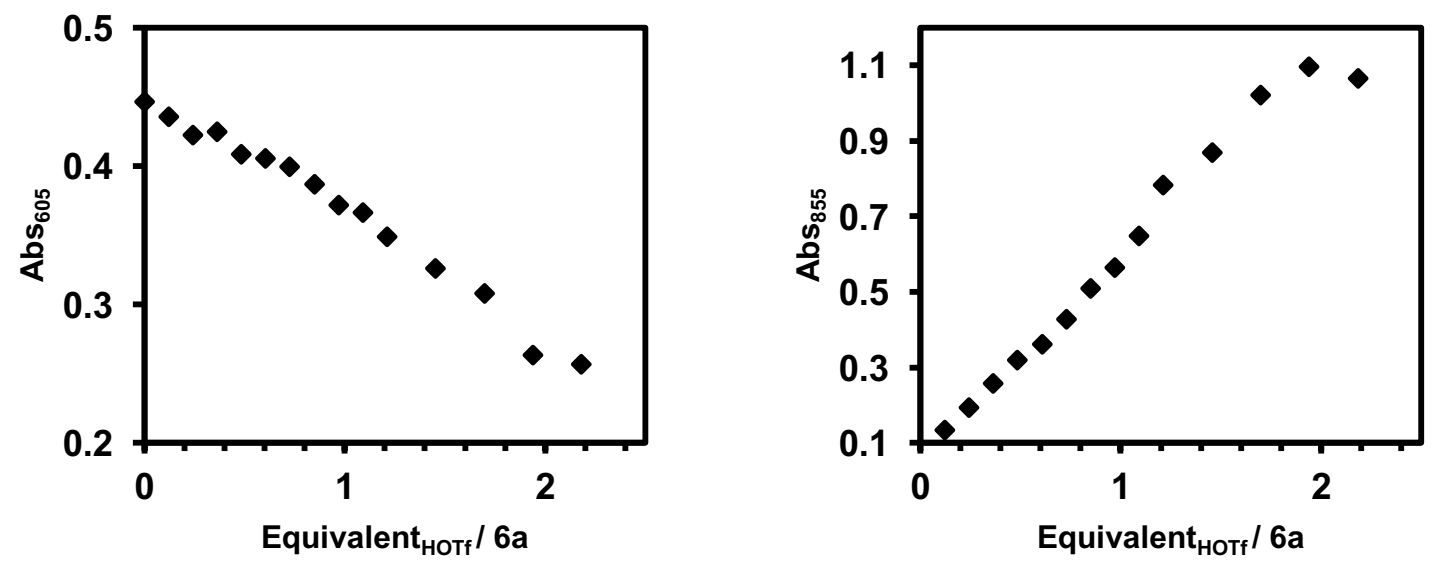

Figure S16. Following the addition of HOTf tracked by the change in absorbances at $605 \mathrm{~nm}$ (left) and $855 \mathrm{~nm}$ (right). After the addition of nearly 1.96 equiv. HOTf, each change in absorbance begins to level off, indicating the endpoint of the reaction. Additional equivalents of acid irreversibly degrade the protonated compound $\mathbf{8 a}$. 

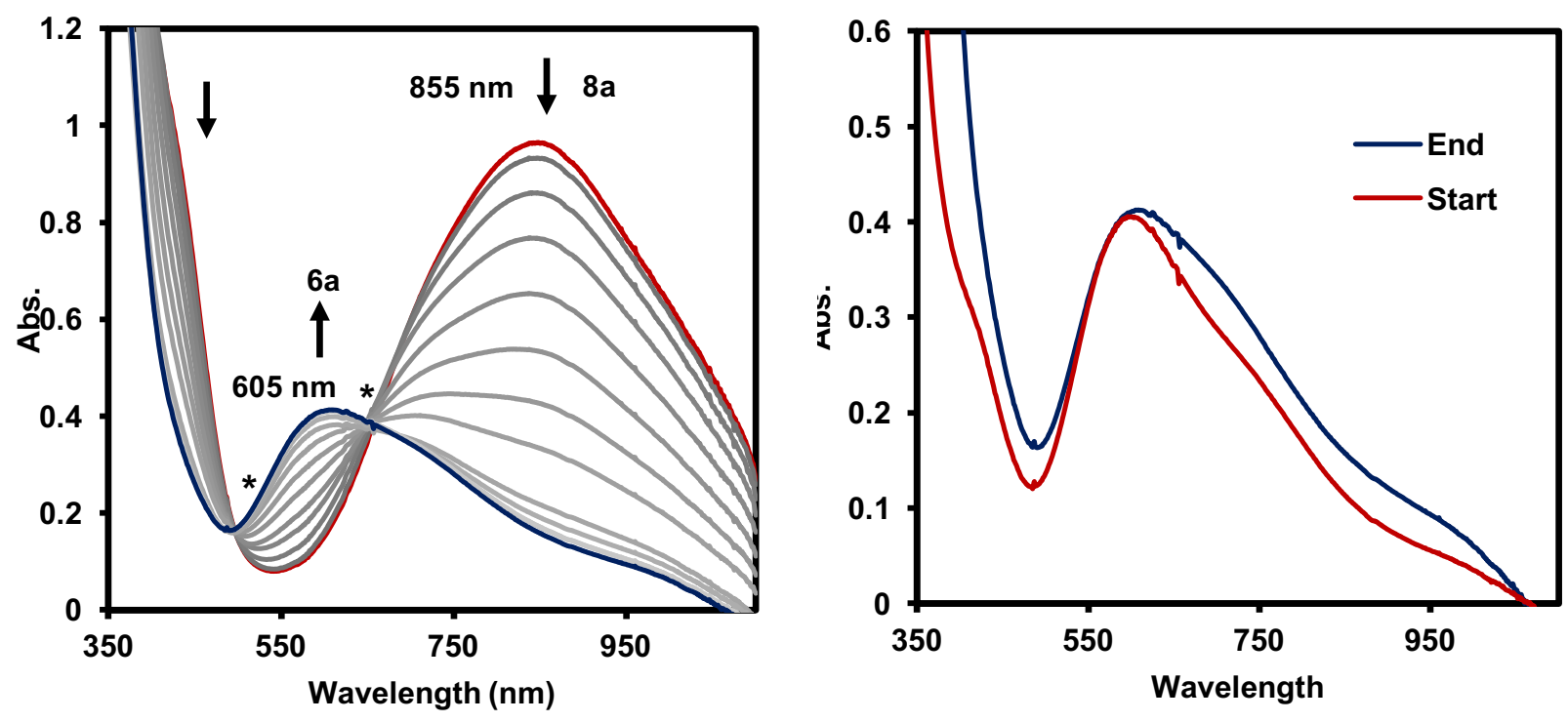

Figure S17. The backtitration of $8 \mathbf{a}$ (formed in situ by addition of 2 equiv. HOTf to the titration/backtitration) at $-10{ }^{\circ} \mathrm{C}$ with $\mathrm{Et}_{3} \mathrm{~N}$ (left). The red trace corresponds to 8a, each gray trace reflects the addition of each portion of $\mathrm{Et}_{3} \mathrm{~N}$ (ca. 0.2 equiv. each), and the blue trace corresponds to the endpoint of the titration to regenerate 6a. Overlaying traces of $\mathbf{6 a}$ before HOTf titration (red) and after full backtitration with $\mathrm{Et}_{3} \mathrm{~N}$ show minor deviations that may reflect minor amounts of degradation of compound 6a during the titration/backtitration sequence (right).
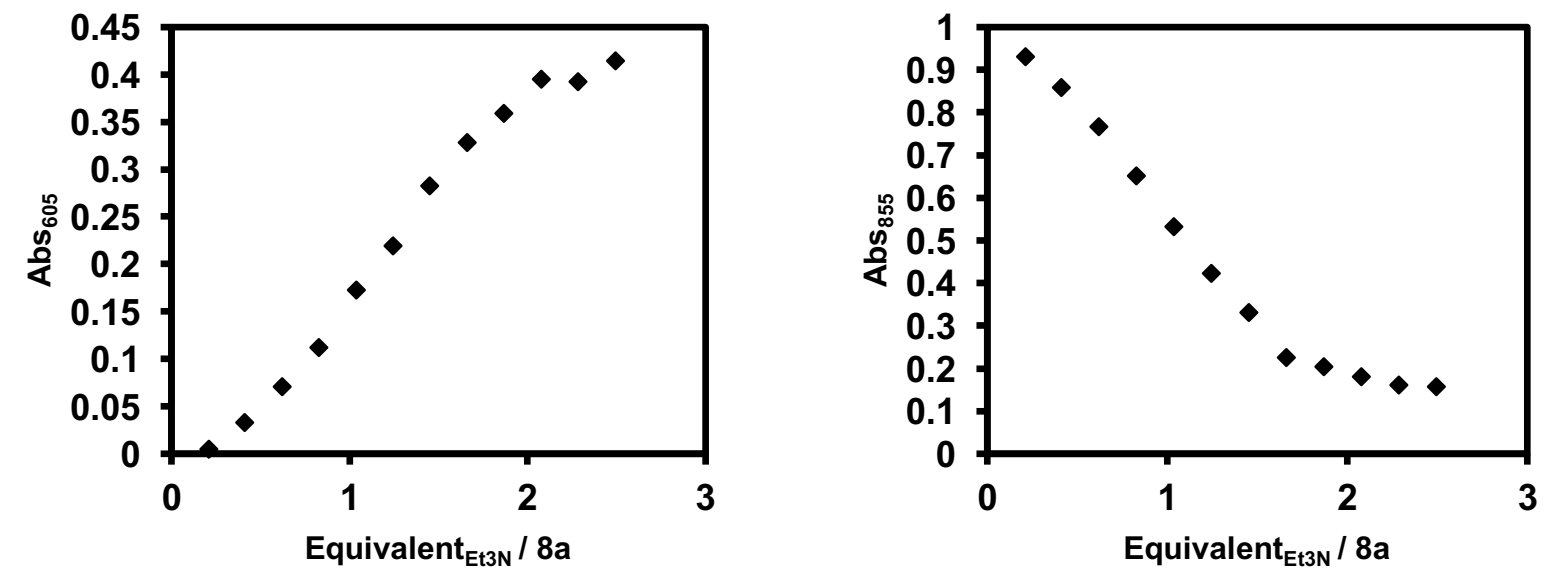

Figure S18. Added equivalents of $\mathrm{Et}_{3} \mathrm{~N}$ tracked by the change in absorbances at $605 \mathrm{~nm}$ (left) and 855 $\mathrm{nm}$ (right) that correspond to $\mathbf{6 a}$ and $\mathbf{8 a}$, respectively. After the addition of ca. 2 equiv. $\mathrm{Et}_{3} \mathrm{~N}$ the conversion of $\mathbf{8 a}$ to $\mathbf{6 a}$ is essentially complete. 


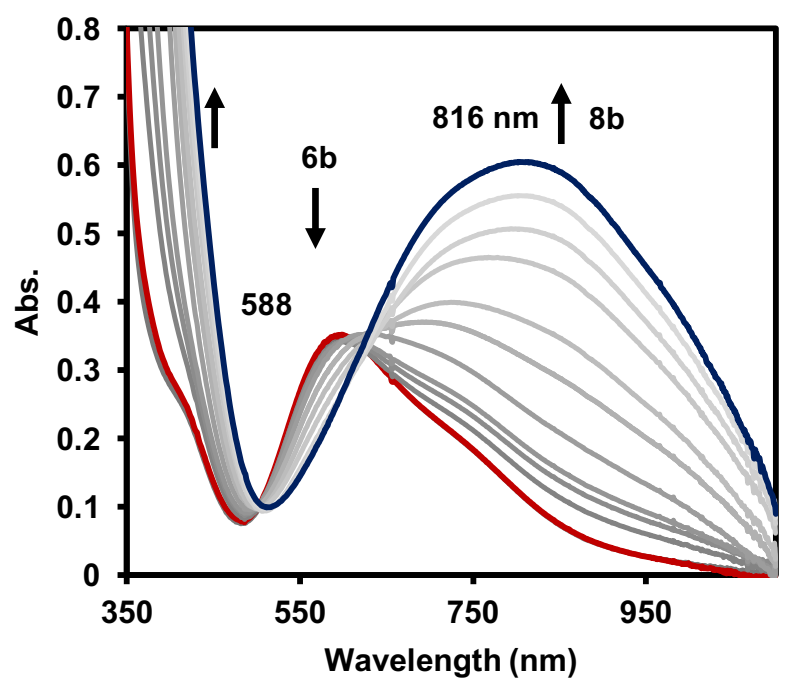

Figure S19. The titration of $\left[{ }^{\mathrm{pymMe}} \mathrm{TpCu}\right]_{2}(\mu-\mathrm{OH})_{2}(\mathbf{6 b})$ by HOTf at $-10{ }^{\circ} \mathrm{C}$. The red trace represents the initial spectrum of $\mathbf{6 b}$, gray traces correspond to the addition of each portion of HOTf (ca. 0.2 equiv. each), and the blue trace correspond to $\mathbf{8 b}$ formed at the endpoint.
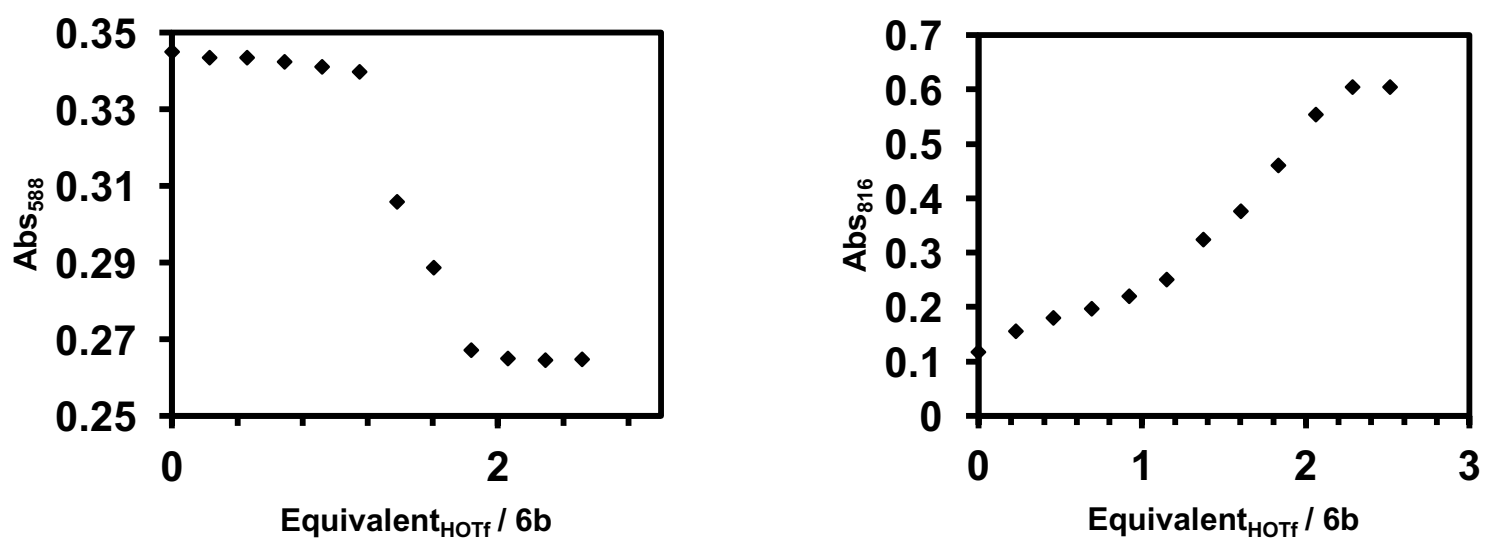

Figure S20. The added equivalents of HOTf tracked by the change in absorbances at $588 \mathrm{~nm}$ (left) and $816 \mathrm{~nm}$ (right). After the addition of nearly ca. 2 equivalents of HOTf, the change in absorbance begins to level off, indicating the endpoint of the reaction. Additional equivalents of acid irreversibly degrade compound $\mathbf{8 b}$. 

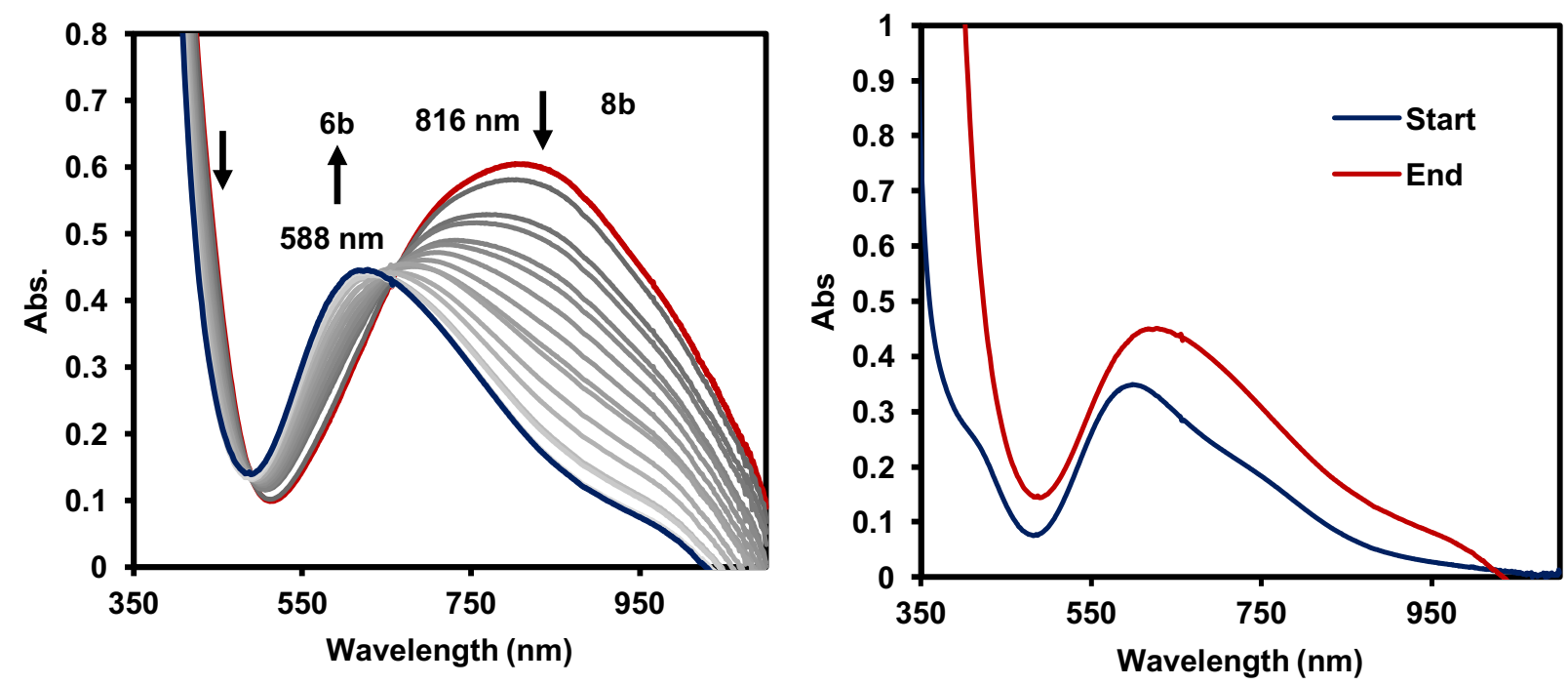

Figure S21. (Left) The backtitration of $\mathbf{8 b}$ formed in situ at $-10{ }^{\circ} \mathrm{C}$ with $\mathrm{Et}_{3} \mathrm{~N}$. The red trace corresponds to $\mathbf{8 b}$ formed in situ at $-10{ }^{\circ} \mathrm{C}$, each gray trace reflects the addition of each portion of $\mathrm{Et}_{3} \mathrm{~N}$ (ca. 0.1 equiv. each), and the blue trace corresponds to the endpoint of the titration to regenerate $6 \mathbf{b}$. (Right) Overlaying traces of $\mathbf{6} \mathbf{b}$ before HOTf titration (red) and after full backtitration with $\mathrm{Et}_{3} \mathrm{~N}$ show deviations that may reflect degradation of compound $\mathbf{6 b}$ during the titration/backtitration sequence (right).
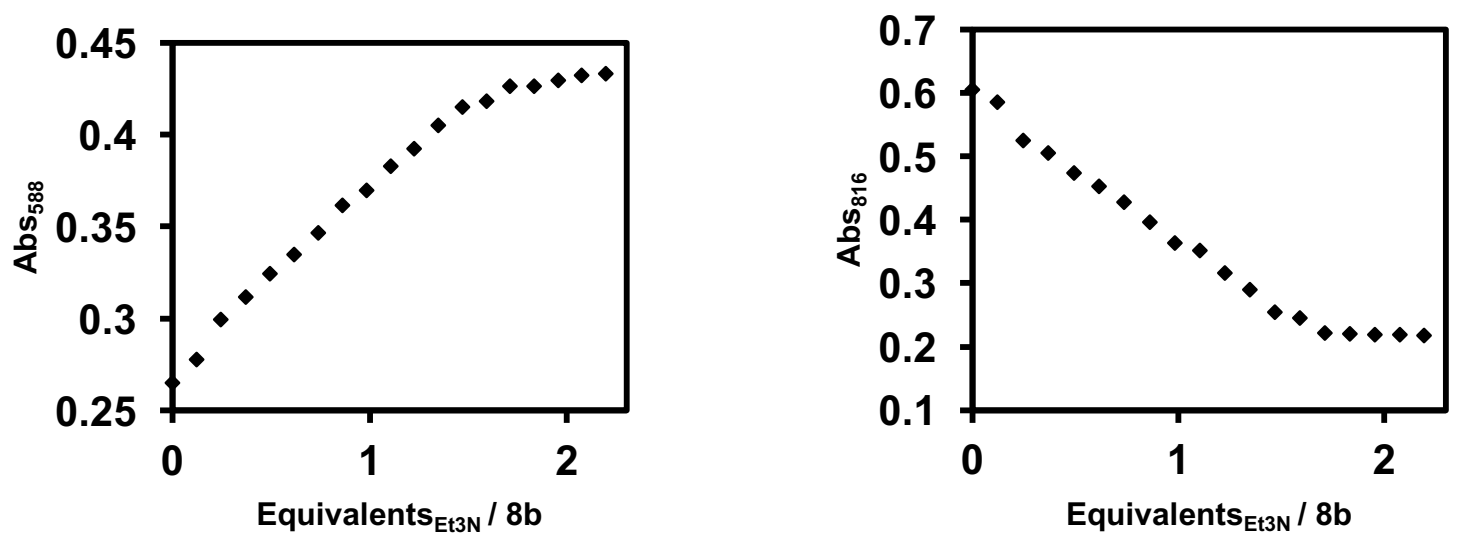

Figure S22. The added equivalents of $\mathrm{Et}_{3} \mathrm{~N}$ tracked by the change in absorbances at $588 \mathrm{~nm}$ (left) and $816 \mathrm{~nm}$ (right). After the addition of ca. 2 equivalents of $\mathrm{Et}_{3} \mathrm{~N}$ the conversion of $\mathbf{8 b}$ to $\mathbf{6} \mathbf{b}$ is nearly complete. 


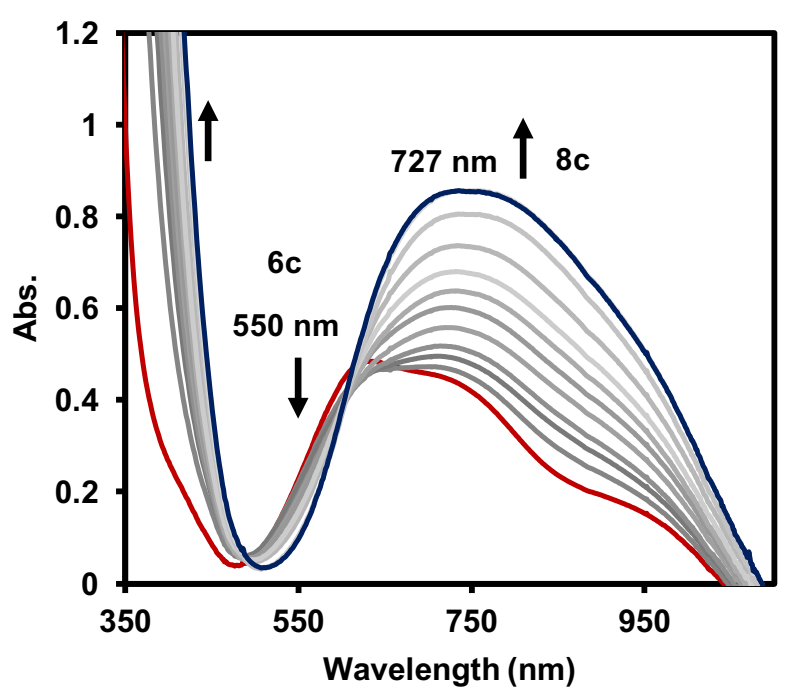

Figure S23. The titration of $\left[{ }^{\mathrm{pyMe}} \mathrm{TpCu}\right]_{2}(\mu-\mathrm{OH})_{2}(\mathbf{6 c})$ by HOTf at $-10{ }^{\circ} \mathrm{C}$. The red trace represents the initial spectrum of $\mathbf{6 c}$, grey traces correspond to species present after each addition of titrant HOTf (ca. 0.2 equiv. each), and the blue trace correspond to $8 \mathbf{c}$ formed at the endpoint.
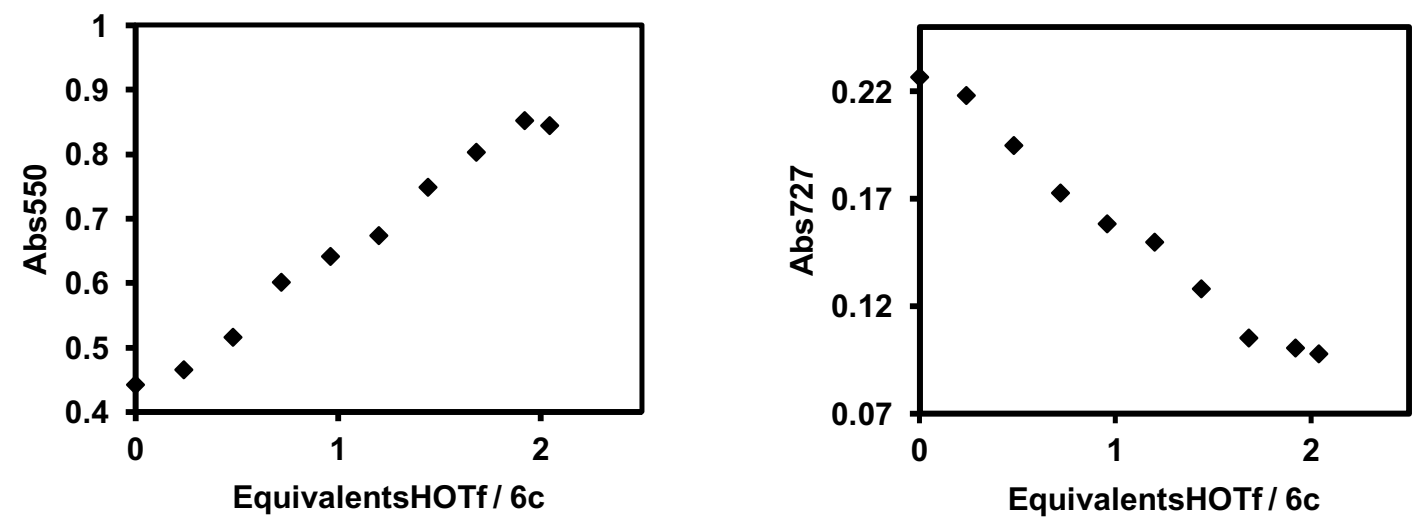

Figure S24. The added equivalents of HOTf tracked by the change in absorbances at $588 \mathrm{~nm}$ (left) and $816 \mathrm{~nm}$ (right). After the addition of nearly ca. 2 equivalents of HOTf, the change in absorbance begins to level off, indicating the endpoint of the reaction. Additional equivalents of acid irreversibly degrade compound $\mathbf{8 b}$. 

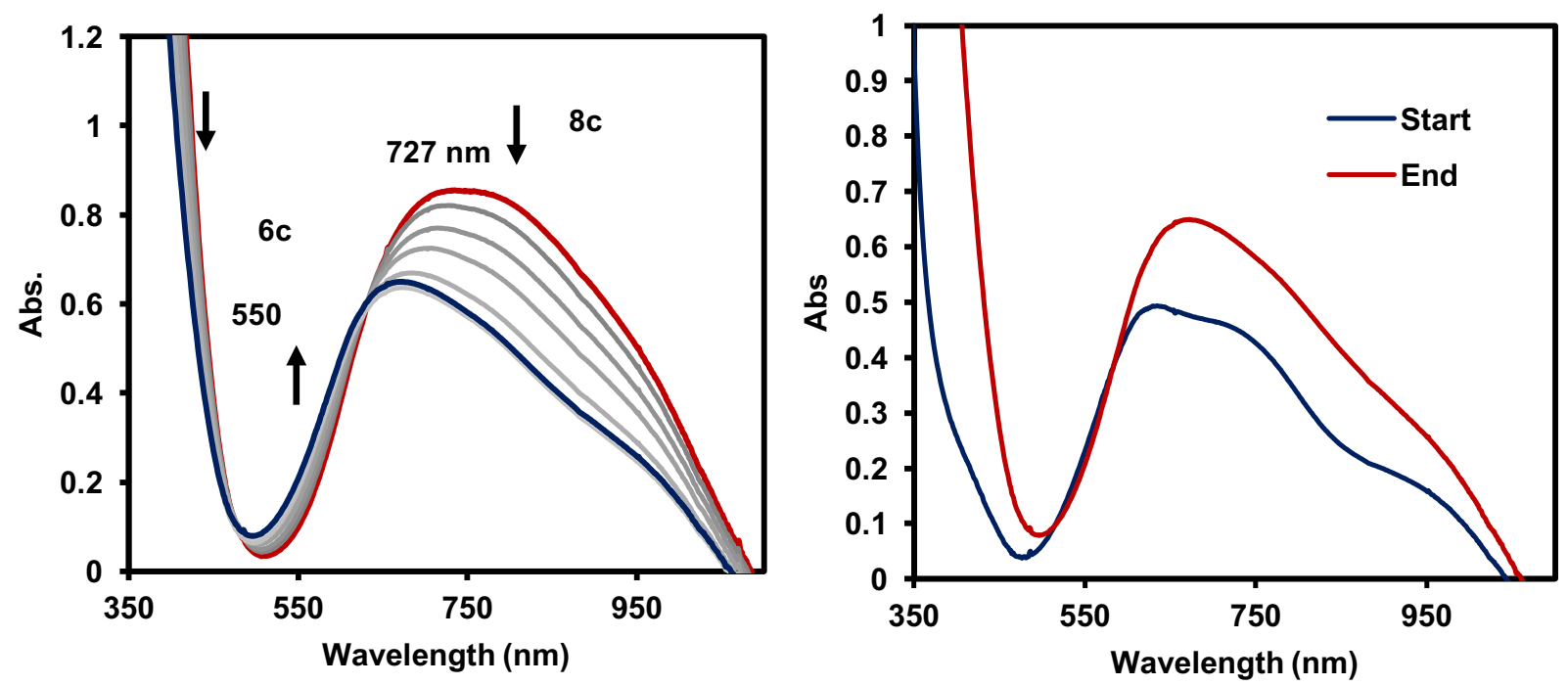

Figure S25. (Left) The backtitration of 8c formed in situ at $-10{ }^{\circ} \mathrm{C}$ with DBU. The red trace corresponds to 8c formed in situ at $-10{ }^{\circ} \mathrm{C}$, each grey trace corresponds to species formed after each addition of DBU (ca. 0.1 equiv. each), and the blue trace corresponds to the endpoint of the titration to regenerate 6c. (Right) Overlaying traces of $\mathbf{6 c}$ before HOTf titration (red) and after full backtitration with DBU show deviations that may reflect the degradation of compound $\mathbf{6 c}$ during the titration/backtitration sequence (right).
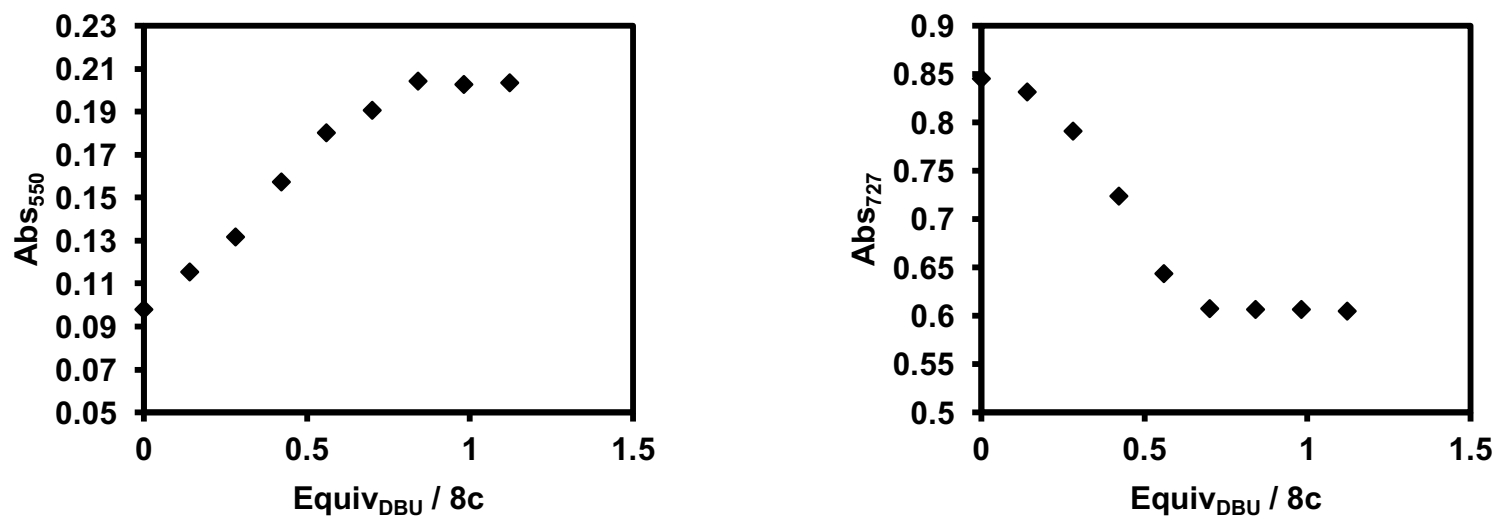

Figure S26. The added equivalents of DBU tracked by the change in absorbances at $550 \mathrm{~nm}$ (left) and $727 \mathrm{~nm}$ (right). After the addition of ca. 1 equivalents of DBU the conversion of $\mathbf{8 c}$ to $\mathbf{6 c}$ is nearly complete. The requirement for 1 equivalent of DBU indicates that DBU is a sufficiently stronger base than the corresponding $\mathrm{OH}^{-}$that is formed. 


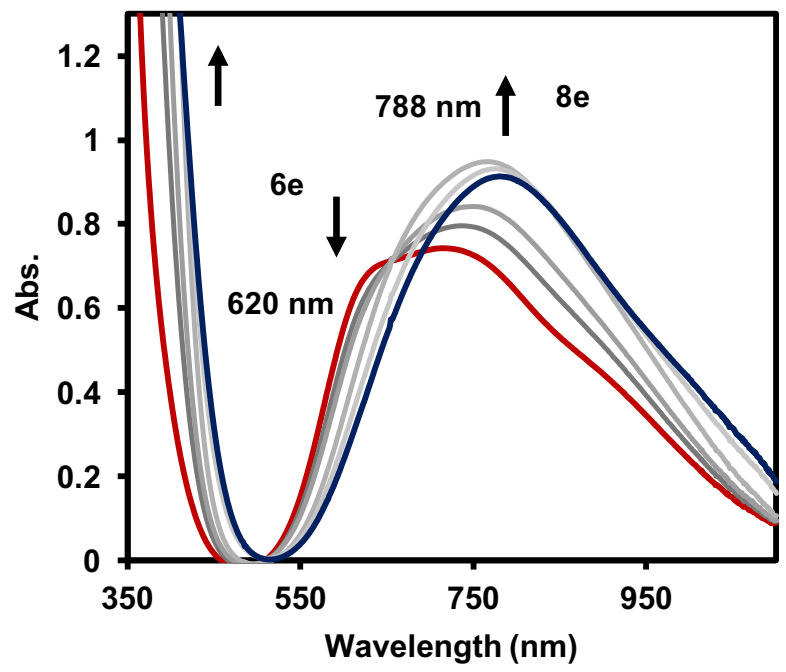

Figure S27. The titration of $\left[{ }^{\mathrm{DMAPMe}} \mathrm{TpCu}\right]_{2}(\mu-\mathrm{OH})_{2}(\mathbf{6 e})$ by HOTf at $-10{ }^{\circ} \mathrm{C}$. The red trace represents the initial spectrum of $\mathbf{6 e}$, grey traces correspond to species present after each addition of titrant HOTf (ca. 0.1 equiv. each), and the blue trace correspond to $\mathbf{8 e}$ formed at the endpoint.
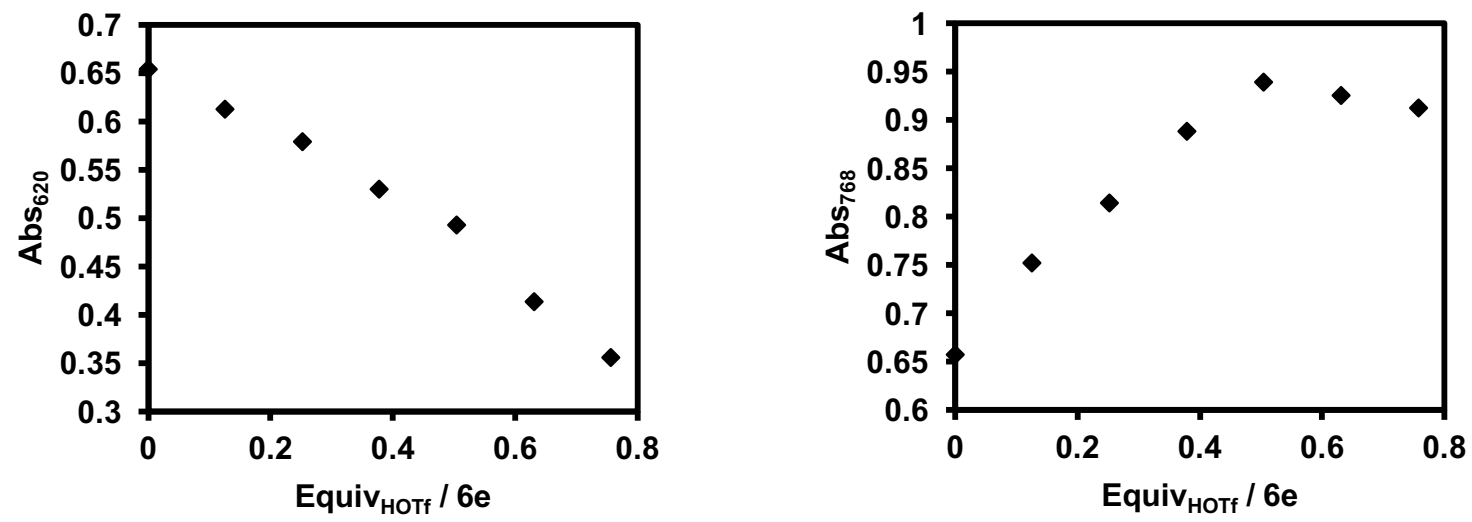

Figure S28. The added equivalents of HOTf tracked by the change in absorbances at $620 \mathrm{~nm}$ (left) and $786 \mathrm{~nm}$ (right). After the addition of nearly ca. 0.8 equivalents of HOTf, the change in absorbance begins to level off, indicating the endpoint of the reaction. Additional equivalents of acid irreversibly degrade compound $\mathbf{8 e}$. Full protonation after one equivalent of HOTf indicates that $\mathbf{6 e}$ is a monomer, as dimers $\mathbf{6 a}-\mathbf{6 c}$ require 2 equivalents of HOTf. 

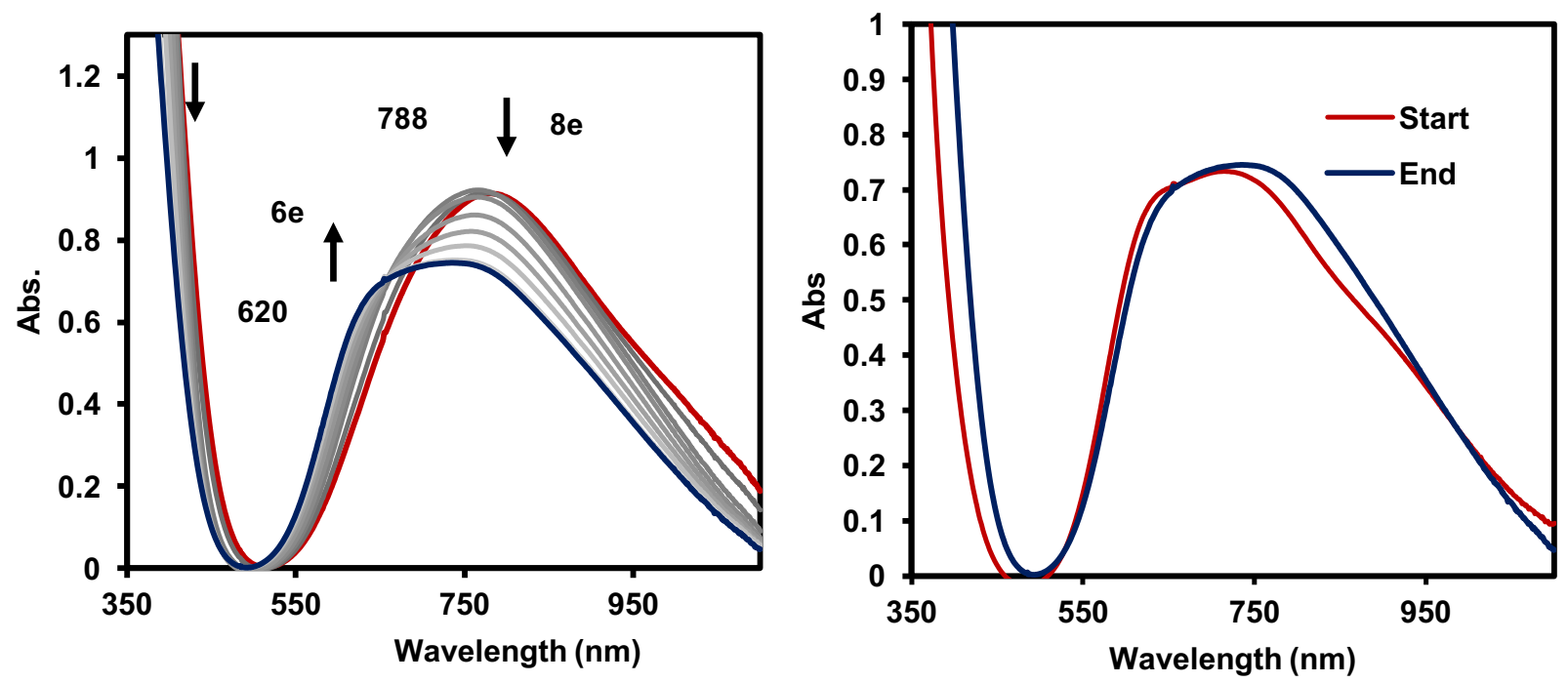

Figure S29. (Left) The backtitration of 8e formed in situ at $-10{ }^{\circ} \mathrm{C}$ with $\mathrm{DBU}$. The red trace corresponds to 8e formed in situ at $-10{ }^{\circ} \mathrm{C}$, each grey trace corresponds to species formed after each addition of DBU (ca. 0.1 equiv. each), and the blue trace corresponds to the endpoint of the titration to regenerate 6e. Overlaying traces of $\mathbf{6 e}$ before HOTf titration (red) and after full backtitration with DBU show minor deviations that may reflect minor amounts of degradation of compound 6e during the titration/backtitration sequence (right).
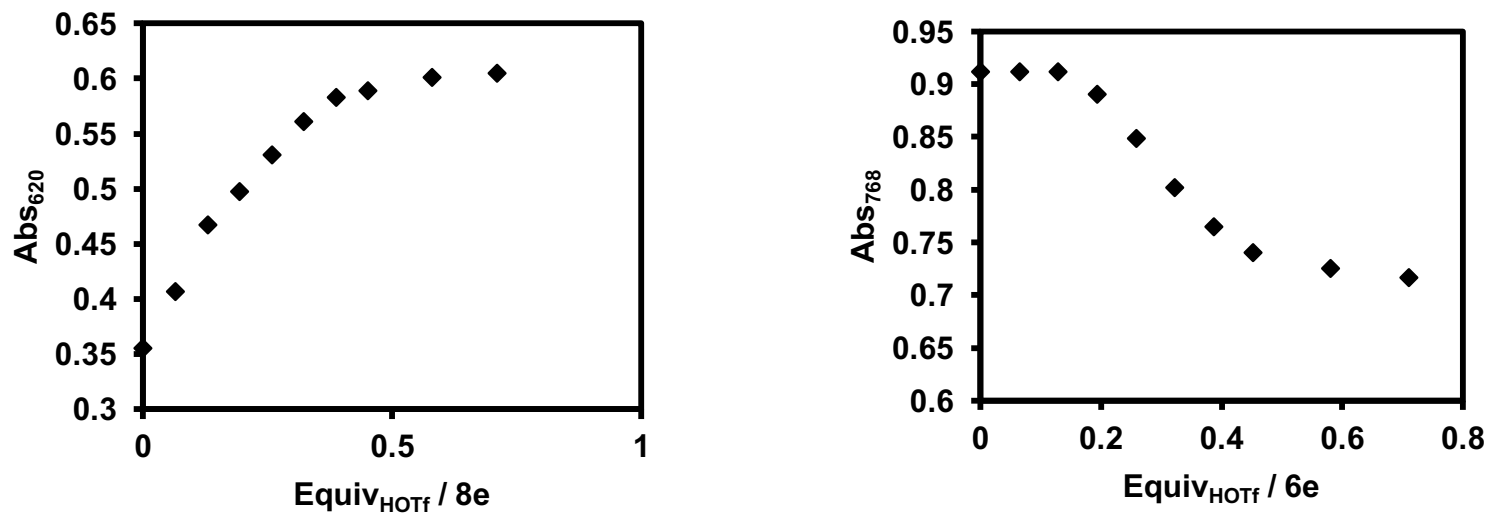

Figure S30. The added equivalents of DBU tracked by the change in absorbances at $620 \mathrm{~nm}$ (left) and $768 \mathrm{~nm}$ (right). After the addition of ca. 0.8 equivalents of DBU the conversion of $8 \mathbf{e}$ to $\mathbf{6 e}$ is nearly complete. The requirement for nearly 0.8 equivalents of DBU indicates that DBU is a sufficiently stronger base than the corresponding $\mathrm{OH}^{-}$that is formed. 
IR Characterization of $8 \mathrm{a}$ and $8 \mathrm{e}$.
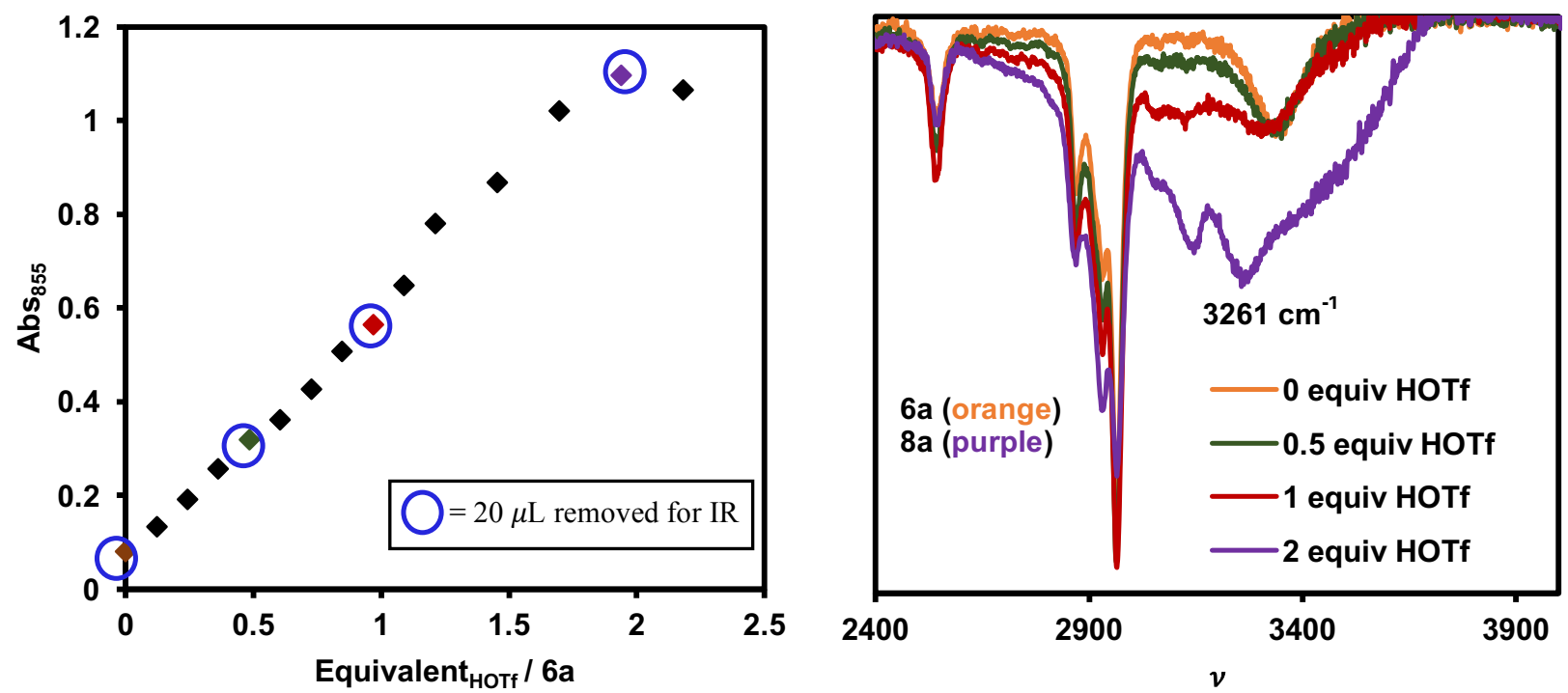

Figure S31. (Left) The growth of the $855 \mathrm{~nm}$ band of 8a relative vs. equivalents of HOTf added to $\left[{ }^{\mathrm{CF} 3 \mathrm{pyMe}} \mathrm{TpCu}\right]_{2}(\mu-\mathrm{OH})_{2}(\mathbf{6 a})$ at $-40{ }^{\circ} \mathrm{C}$ tracked by UV-vis. $20 \mu \mathrm{L}$ aliquots of the solution were removed via a chilled Hamilton syringe at $0,0.5,1$, and 2 equiv. HOTf, which is indicated by a blue circle. These aliquots were then evaporated under a stream of $\mathrm{N}_{2}$ on an ATR-IR probe. The corresponding IR spectra (right) taken at these equivalences reveal the steady formation of broad band centered around $3261 \mathrm{~cm}^{-1}$, corresponding to an aquo ligand formed in situ.
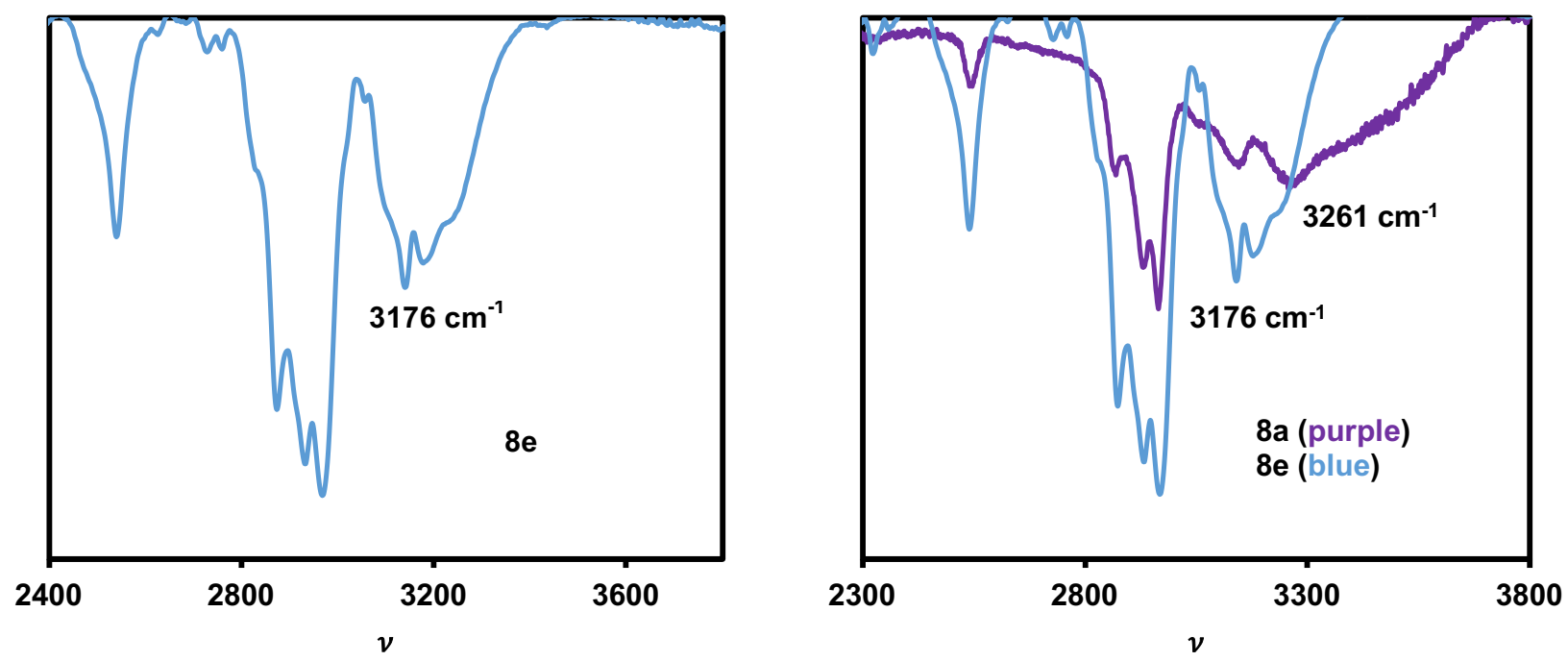

Figure S32. (Left) Thin film IR of 8e, which was evaporated from DCM. 8e was synthesized from protonation of $6 \mathbf{e}$ with one equivalent of HOTf in DCM at $-40{ }^{\circ} \mathrm{C}$. The broad band exhibited by $8 \mathbf{e}$ centered around $3176 \mathrm{~cm}^{-1}$ is distinct from the $\mathrm{OH}^{-}$vibration of the precursor 6e, supporting the formation of an aquo complex. (Right) IR overlay of $\mathbf{8 e}$ (blue) and $\mathbf{8 a}$ (purple) indicate broad bands that can reasonably be assigned to an aquo ligand. The activation of $v_{\mathrm{HO}-\mathrm{H}}$ is greater in $\mathbf{8 e}$ than in $\mathbf{8 a}$, which suggests a stronger $\mathrm{H}$-bond contribution from a pendant DMAP in comparison to ${ }^{\mathrm{CF} 3} \mathrm{py}$. A similar trend is observed in the corresponding hydroxide complexes $\mathbf{6 a}-\mathbf{6 e}$ (Figure S10). 


\section{X-ray Refinement Details}

Single crystals of each compound 3c (CCDC 1890609), 3e (CCDC 1890610), 4a (CCDC 1890611), 4c (CCDC 1890612), 5b (CCDC 1890613), 5c (CCDC 1890614), 6a (CCDC 1890615), 6b (CCDC 1890616), 6c (CCDC 1890617), and 6e (CCDC 1890618) were mounted under mineral oil on a Mitegen micromount and immediately placed in a cold nitrogen stream at 100(2) K prior to data collection. Data for compounds 3c, 3e, 4a, 4c, 5b, and $\mathbf{6 b}$ were collected on a Bruker D8 Quest equipped with a Photon100 CMOS detector and a Mo ImS source. Data for compounds 5c, 6a, $\mathbf{6 c}$, and $\mathbf{6 e}$ were collected on a Bruker DUO equipped with an APEXII CCD detector and Mo fine-focus sealed source. Either full spheres (triclinic) or hemispheres (monoclinic or higher) of data were collected $\left(0.3^{\circ}\right.$ or $0.5^{\circ} \omega$-scans; $2 \theta$ $\max =56^{\circ}$; monochromatic Mo K $\alpha$ radiation, $\lambda=0.7107 \AA$ ) depending on the crystal system and integrated with the Bruker SAINT program. Structure solutions and refinements were performed using the SHELX suite $^{\mathrm{a}}$ and SHELXLE. ${ }^{\mathrm{b}}$ Intensities were corrected for Lorentz and polarization effects and an empirical absorption correction was applied using Blessing's method as incorporated into the program SADABS. ${ }^{\mathrm{c}}$ Non-hydrogen atoms were refined with anisotropic thermal parameters. The borate hydrogen atoms were located in the difference map while all other hydrogen atoms were included in idealized positions unless otherwise noted below. Structures were rendered with POV-Ray in Mercury ${ }^{\mathrm{d}}$ using $50 \%$ probability ellipsoids.

\section{Additional Refinement Notes:}

$X$-ray structure of ${ }^{p y M e} \mathrm{PzH}(\mathbf{3 c})$.

The pyrazole hydrogen atom was located in the difference map and its position was allowed to freely refine. It refined to a good intermolecular hydrogen bonding position.

$X$-ray structure of ${ }^{\text {DMAPMe }} \mathrm{PzH}(\mathbf{3 e})$.

There are two unique molecules in the asymmetric unit. The pyrazole hydrogen atom was located in the difference map for each molecule. The $\mathrm{N}-\mathrm{H}$ distances were restrained to be $0.88(2) \AA$. Both $\mathrm{H}$ atoms refine to good intermolecular hydrogen bonding positions. The datum crystal exhibited pseudomerohedral twinning. The two twin domains are related by the twin law [ $\left[\begin{array}{lll}0 & 0 & -1\end{array}\right]\left[\begin{array}{lll}0 & -1 & 0\end{array}\right]\left[\begin{array}{lll}-1 & 0 & 0\end{array}\right]$ and the ratio refines to $78: 22$.

$X$-ray structure of ${ }^{C F 3 p y M e} T p K$ (4a).

The fluorine atoms of the $\mathrm{CF}_{3}$ group are disordered over two positions. The $\mathrm{C}-\mathrm{F}$ and $\mathrm{F} \cdots \mathrm{F}$ distances, respectively, were restrained to be similar. Similar displacement amplitudes were imposed on disordered sites overlapping by less than the sum of van der Waals radii. The borate hydrogen atom was located in the difference map and its position was allowed to freely refine. 


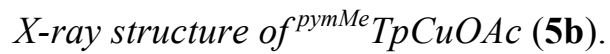

Two of the isopropyl group are disordered over two orientations. In each case, the C-C distances were restrained to be similar. Similar displacement amplitudes were imposed on disordered sites overlapping by less than the sum of van der Waals radii.

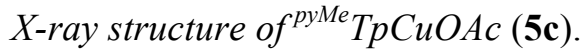

One of the DCM solvent molecules is disordered over positions. The $\mathrm{C}-\mathrm{Cl}$ distances were restrained to be similar. Similar displacement amplitudes were imposed on disordered sites overlapping by less than the sum of van der Waals radii.

$X$-ray structure of $\left[{ }^{\mathrm{CF} 3 p y M e} \mathrm{TpCu}\right]_{2}(\mu-\mathrm{OH})_{2}(\mathbf{6 a})$.

A structural model consisting of the target $\mathrm{Cu}$ dimer complex and four highly disordered DCM solvent molecules was developed. Attempts to model the disordered solvent molecules were unsuccessful, so they were removed from the model using the 'squeeze' routine in PLATON. ${ }^{\mathrm{e}}$ Both $\mathrm{CF}_{3}$ groups are disordered over two orientations. The C-C and C-F distances, respectively, were restrained to be similar. F3 and F3B were restrained to behave relatively isotropic. Similar displacement amplitudes were imposed on disordered sites overlapping by less than the sum of van der Waals radii. The hydroxyl hydrogen atoms were located in the difference map. The O-H distances were restrained to be 0.84(1) $\AA$. Both hydrogen atoms refine to good intramolecular hydrogen bonding positions.

$X$-ray structure of $\left[^{p y m M e} \mathrm{TpCu}\right]_{2}(\mu-\mathrm{OH})_{2}(\mathbf{6 b})$.

A structural model consisting of the target $\mathrm{Cu}$ dimer complex and three highly disordered DCM solvent molecules was developed. Attempts to model the disordered solvent molecules were unsuccessful, so they were removed from the model using the 'squeeze' routine in PLATON. ${ }^{\mathrm{e}}$ Two of the isopropyl group are disordered over two orientations. In each case, the $\mathrm{C}-\mathrm{C}$ distances were restrained to be similar. Similar displacement amplitudes were imposed on disordered sites overlapping by less than the sum of van der Waals radii. The hydroxyl hydrogen atoms were located in the difference map. The O-H distances were restrained to be $0.84(1) \AA$. Both hydrogen atoms refine to good intramolecular hydrogen bonding positions.

$X$-ray structure of $\left[^{p y M e} \mathrm{TpCu}\right]_{2}(\mu-\mathrm{OH})_{2}(\mathbf{6 c})$.

A structural model consisting of one half of the target molecule and two DCM solvent molecules per asymmetric unit was developed. Both solvent molecules are disordered over two positions. The $\mathrm{C}-\mathrm{Cl}$ distances were restrained to be similar. Rigid-bond restraints were imposed on displacement parameters for all disordered sites and similar displacement amplitudes were imposed on disordered sites overlapping by less than the sum of van der Waals radii. The hydroxyl hydrogen atom was located in the difference map. The O-H distance was restrained to be 0.84(1) $\AA$. 
$X$-ray structure of ${ }^{D M A P M e} \mathrm{Tp} C \mathrm{Cu} \mathrm{OH}(\mathbf{6 e})$.

One of the three DCM solvent molecules is disordered over two orientations. The C-Cl distances were restrained to be similar. The disordered atoms were restrained to behave relatively isotropic. Similar displacement amplitudes were imposed on disordered sites overlapping by less than the sum of van der Waals radii. The hydroxyl hydrogen atom was located in the difference map. The O-H distance was

restrained to be $0.84(1) \AA$. There is no hydrogen bond acceptor available for interaction with the hydroxyl $\mathrm{H}$ atom. 


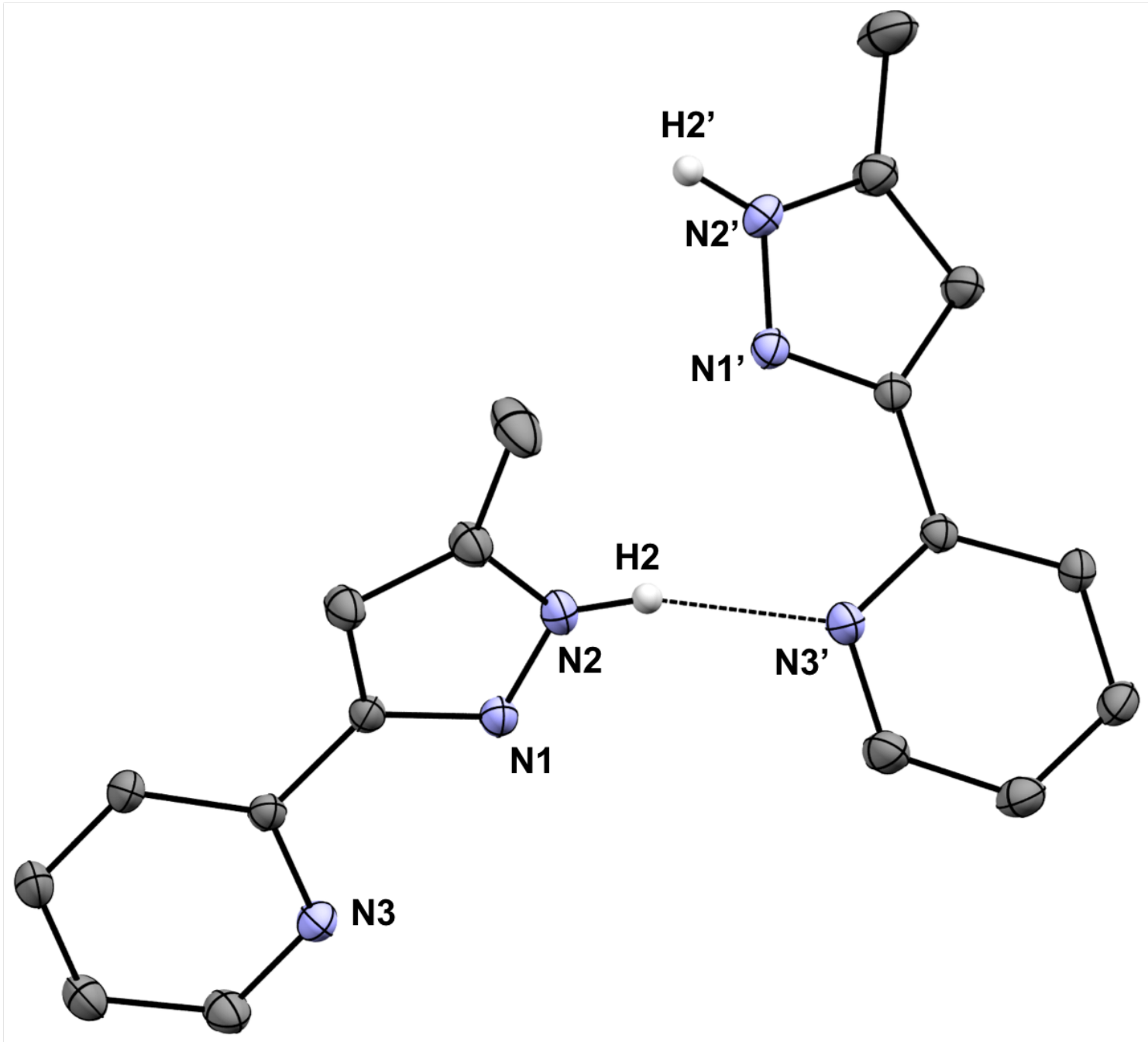

Figure S10. X-ray structure of ${ }^{\text {pyMe }} \mathrm{PzH}$ (3c) (CCDC 1890609, two repeating H-bonding units represented with all but select $\mathrm{H}$ atoms omitted; thermal ellipsoids are represented at the 50\% probability level). Select bond distances $(\AA)$ and angles $\left(^{\circ}\right)$ : N3'-(H2)N2 2.8609(14), N3-H2-N2 163.9(18). 


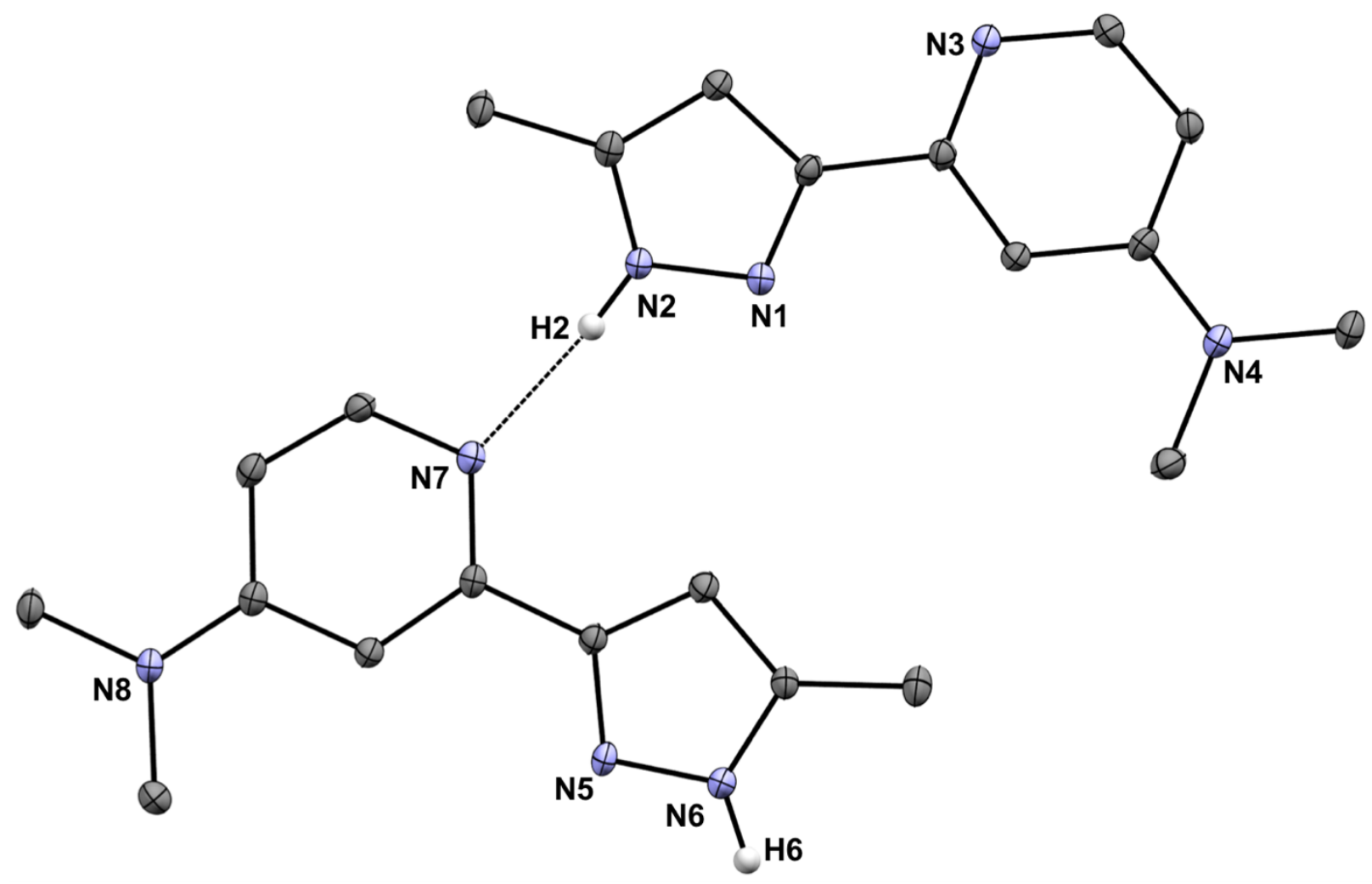

Figure S11. X-ray structure of ${ }^{\text {DMAPMe }} \mathrm{PzH}$ (3e) (CCDC 1890610). The H-bonding units represented with all but select $\mathrm{H}$ atoms omitted. The thermal ellipsoids are represented at the $50 \%$ probability level. Selected bond distance $(\AA)$ and angle $\left(^{\circ}\right)$ : N7-N2 2.902(2), N7-H2-N2 174(2). 


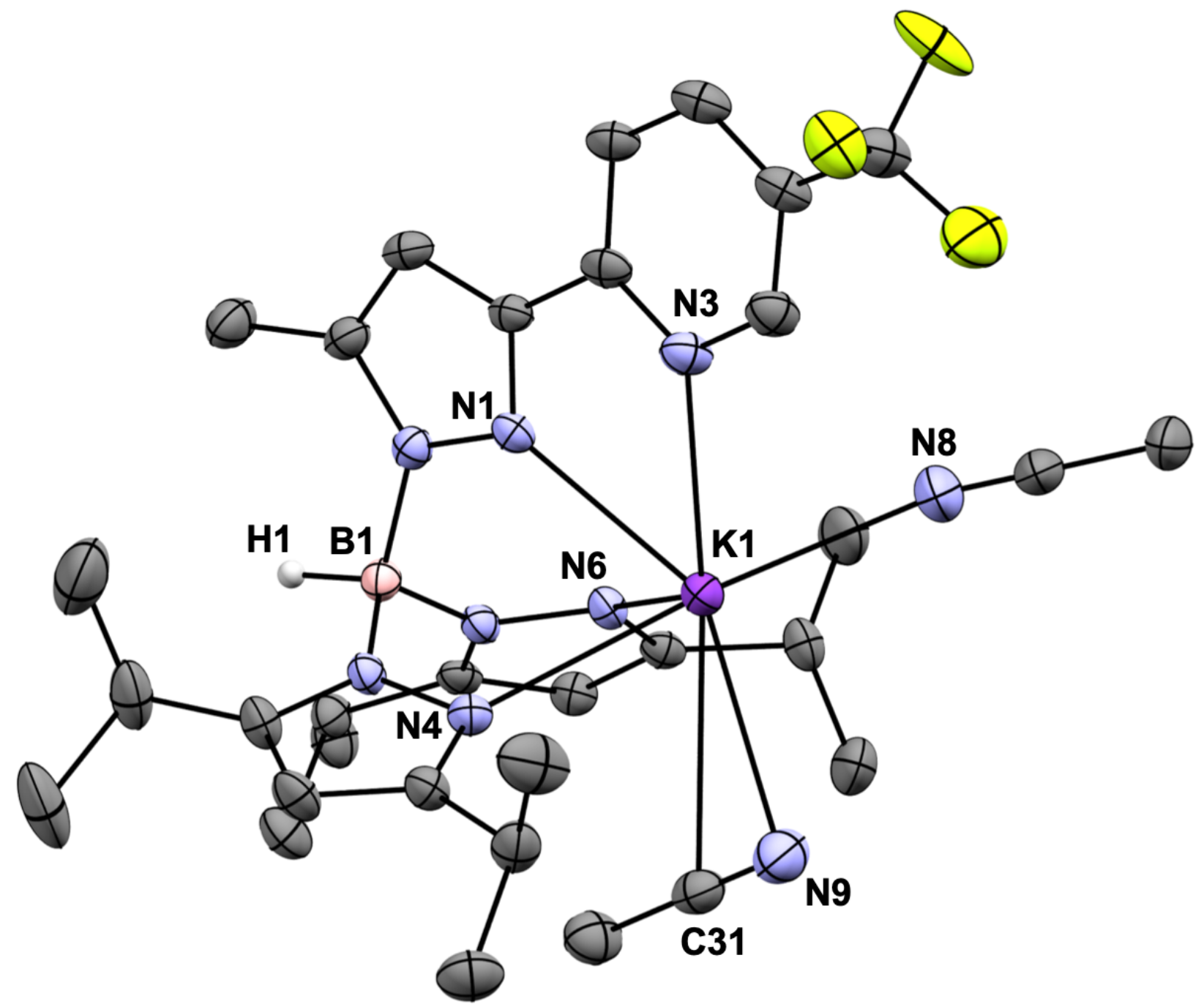

Figure S12. X-ray structure of ${ }^{\text {CF3pyMe }} \mathrm{TpK}$ (4a) (CCDC 1890611). All but selected H atoms omitted. The $\mathrm{CF}_{3}$ group is disordered over two positions and is represented by sites of highest occupancy. The thermal ellipsoids are represented at the 50\% probability level. Selected bond distances $(\AA)$ and angles $\left({ }^{\circ}\right)$ : K1-N1 2.679(2), K1-N3 2.951(2), K1-N4 2.795(2), K1-N6 2.799(2), K1-N8 2.773(3), K1-N9 2.949(3), K1-C31 3.342(3), N1-K1-N3 58.13(6), N1-K1-N4 68.46(6), N1-K1-N6 67.96(6), N4-K1-N6 71.81(6). 


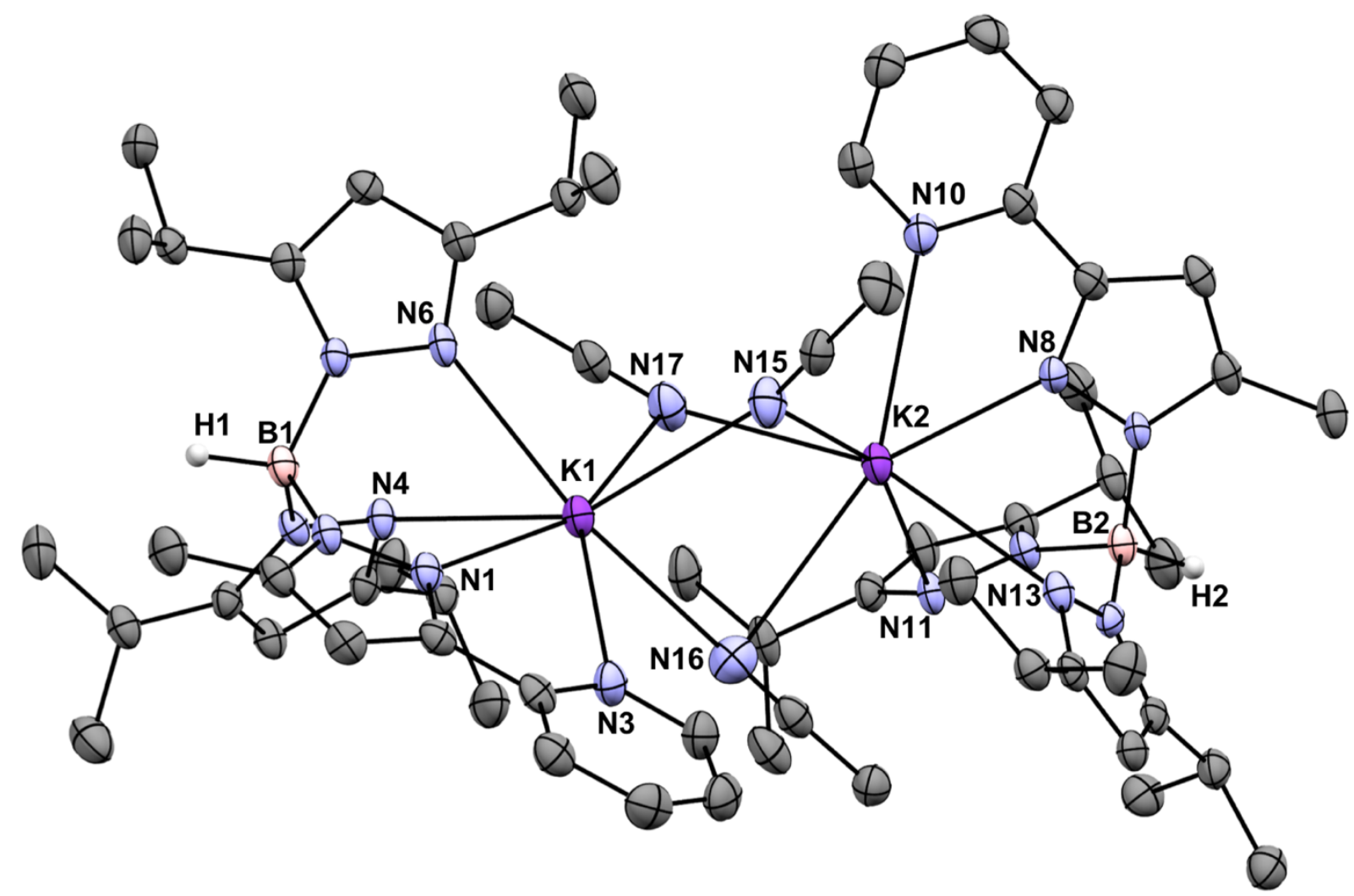

Figure S13. X-ray structure of ${ }^{\text {pyMe }} \mathrm{TpK}$ (4c) (CCDC 1890612). All but selected H atoms and MeCN solvate molecules are omitted. The thermal ellipsoids are represented at the $50 \%$ probability level. Selected bond distances $(\AA)$ and angles $\left(^{\circ}\right)$ : K1-N1 2.699(2), K1-N3 2.958(2), K1-N4 2.922(2), K1-N6 2.863(2), K1-N15 2.912(3), K1-N16 2.958(3), K1-N17 3.067(2), K2-N8 2.700(2), K2-N10 2.990(2), K2N11 2.947(2)，K2-N13 2.896(2)，K2-N15 3.028(3)，K2-N16 3.177(3)，K2-N17 2.849(3)，K1-K2 4.3046(8), N1-K1-N3 58.07(6), N1-K1-N4 72.54(6), N1-K1-N6 68.41(6), N4-K1-N6 66.00(6), N11-K2N13 69.13(6), N8-K2-N11 76.31(6), N8-K2-K13 68.56(6), N8-K2-N10 57.04(6). 


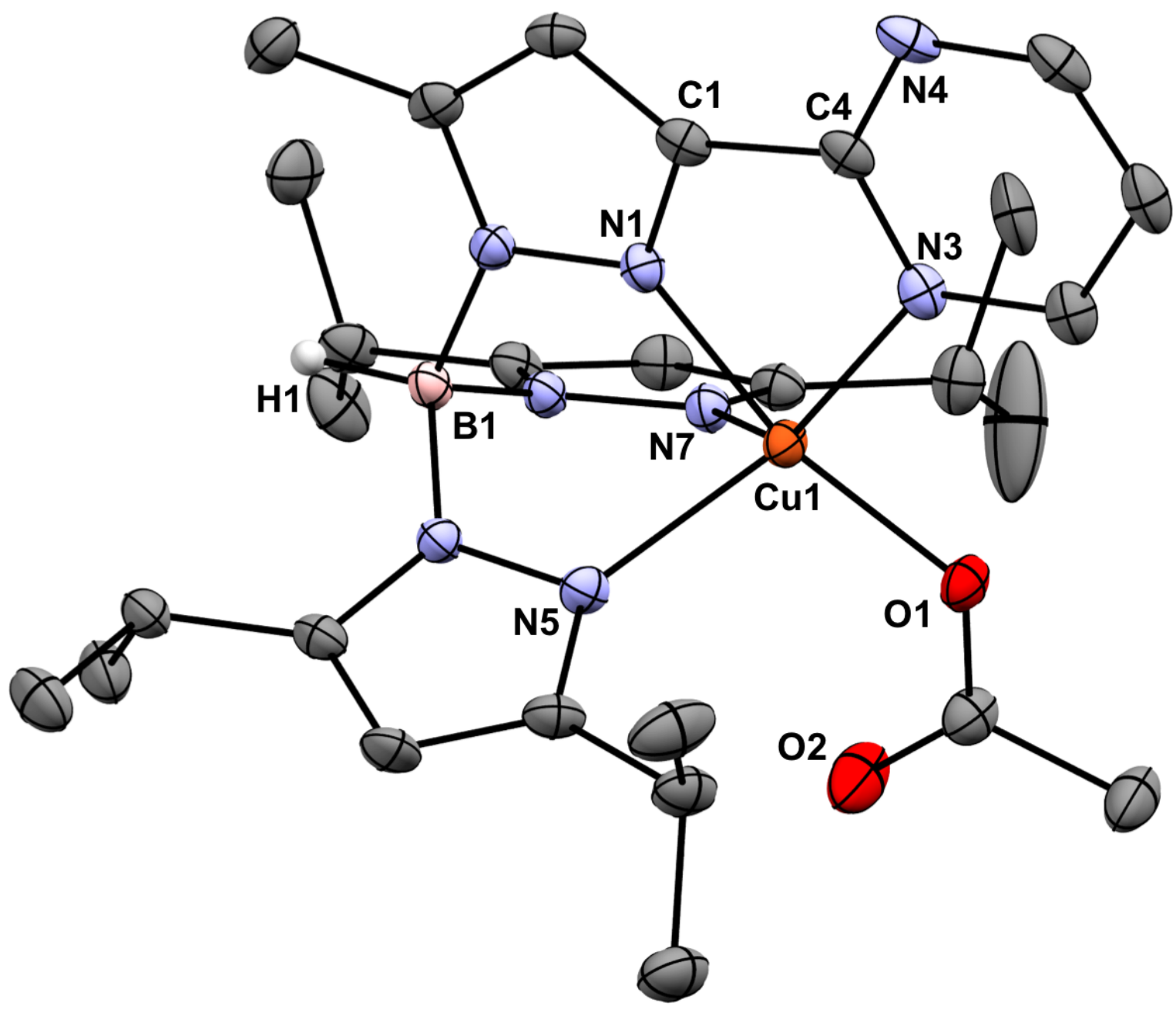

Figure S14. X-ray structure of ${ }^{\text {pymMe }} \mathrm{TpCuOAc}$ (5b) (CCDC 1890613). All but selected $\mathrm{H}$ atoms and a DCM solvate molecule are omitted. Two of the isopropyl groups are disordered over two orientations and are represented by sites of highest occupancy. The thermal ellipsoids are represented at the 50\% probability level. Selected bond distances $(\AA ̊)$ and angles $\left(^{\circ}\right)$ : Cu1-N1 1.9294(19), Cu1-N3 2.1412(18), Cu1-N5 2.1093(19), Cu1-N7 2.1182(18), Cu1-O1 1.9033(16), N1-Cu1-N3 75.91(7), N1-Cu1-N5 85.20(8), N1-Cu1-N7 83.85(8), N5-Cu1-N7 90.57(7), N1-Cu1-O1 164.89(7), N3-Cu1-01 89.07(7), N5Cu1-O1 106.44(7), N7-Cu1-O1 105.20(7), N3-C4-C1 112.81(19). 


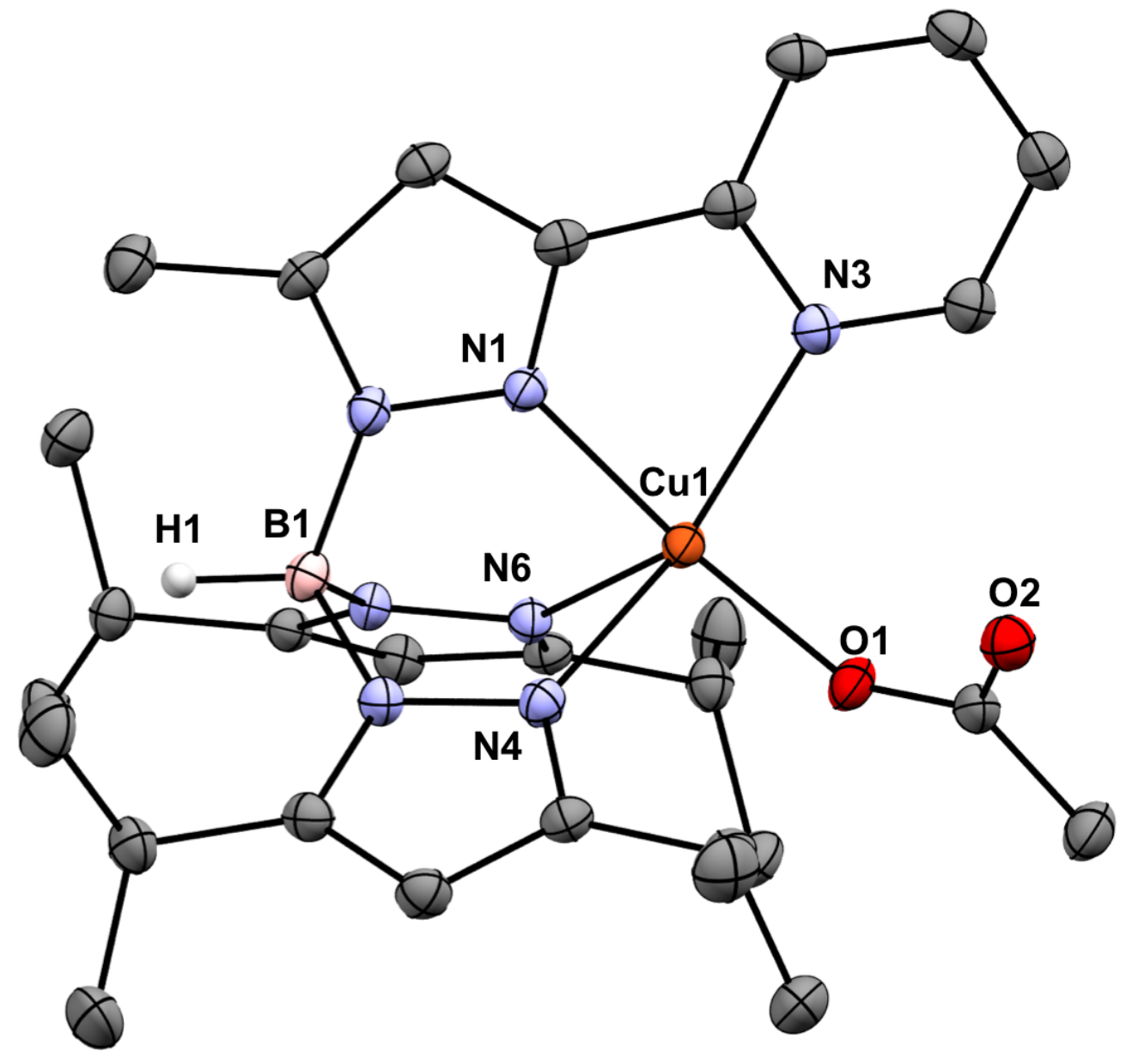

Figure S15. X-ray structure of ${ }^{\text {pyMe }} \mathrm{TpCuOAc}(5 \mathrm{c})$ (CCDC 1890614). All but selected H atoms and DCM solvate molecules are omitted. The thermal ellipsoids are represented at the $50 \%$ probability level. Selected bond distances $(\AA)$ and angles $\left({ }^{\circ}\right)$ : Cu1-N1 1.9225(14), Cu1-N3 2.1454(15), Cu1-N4 2.1240(14), Cu1-N6 2.2208(15), Cu1-O1 1.9010(12), N1-Cu1-N3 76.31(6), N1-Cu1-N4 84.40(6), N1-Cu1-N6 86.93(6), N6-Cu1-N4 85.88(5), N1-Cu1-O1 174.91(6), N3-Cu1-01 98.88(6), N4-Cu1-O1 100.43(6), N6Cu1-O1 95.01(5), N3-C4-C1 113.22(15). 


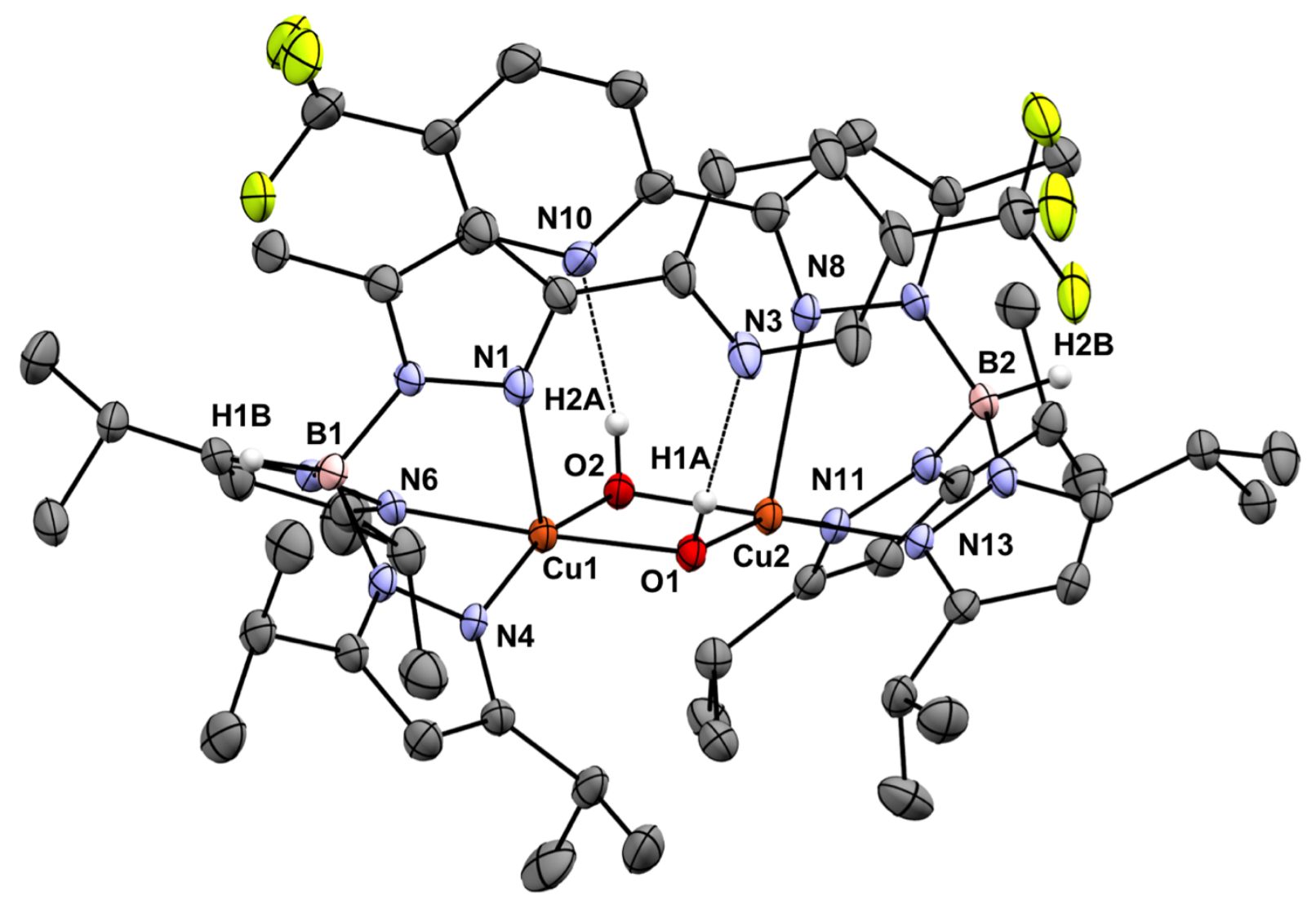

Figure S16. X-ray structure of $\left[{ }^{\mathrm{CF} 3 p y M e} \mathrm{TpCu}\right]_{2}(\mu-\mathrm{OH})_{2}(\mathbf{6 a})(\mathrm{CCDC} 1890615)$. All but selected H atoms omitted. The $\mathrm{CF}_{3}$ groups are disordered over two positions and are represented by sites of highest occupancy. The thermal ellipsoids are represented at the 50\% probability level. Selected bond distances ( $\AA$ ) and angles $\left({ }^{\circ}\right)$ : Cu1-N1 2.5514(33), Cu1-N4 1.991(3), Cu1-N6 2.025(3), N1-Cu1-N4 79.56(12), N1Cu1-N6 92.08(12), N4-Cu1-N6 87.81(12) Cu1-O1 1.943(2), Cu1-O2 1.937(3), O1-O2 2.5365(38), Cu1O1-Cu2 98.49(12), Cu1-O2-Cu2 98.52(12), O1-Cu1-O2 81.65(11), Cu1-O1-H1A 108(3), Cu2-O1-H1A 105(3), Cu1-O2-H2A 110(3), Cu2-O2-H2A 103(3), Cu1-Cu2 2.9457(11), Cu2-N8 2.5514(33), Cu2-N11 1.995(3), Cu2-N13 2.016(3), Cu2-O1 1.946(3), Cu2-O2 1.951(2), N8-Cu2-N11 82.76(12), N8-Cu2-N13 91.51(11), N11-Cu2-N13 86.51(12), O1-Cu2-O2 81.23(11) N3-O1 2.881(4), N10-O2 2.921(4). 


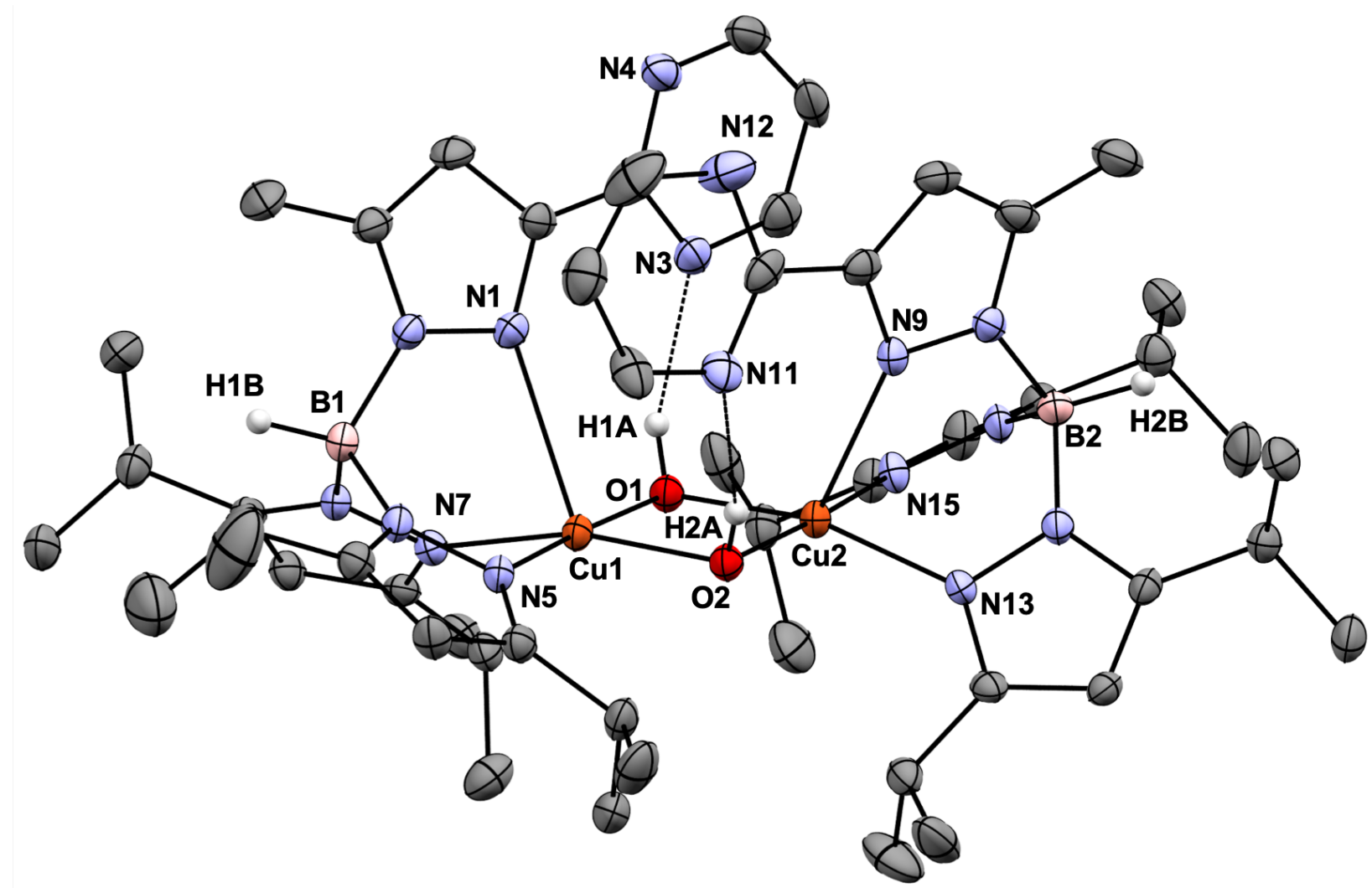

Figure S17. X-ray structure of $\left[{ }^{\mathrm{pymMe}} \mathrm{TpCu}\right]_{2}(\mu-\mathrm{OH})_{2}(\mathbf{6 b})(\mathrm{CCDC} 1890616)$. All but selected $\mathrm{H}$ atoms are omitted. Two of the isopropyl groups are disordered over two orientations and are represented by sites of highest occupancy. The thermal ellipsoids are represented at the $50 \%$ probability level. Selected bond distances $(\AA)$ and angles $\left({ }^{\circ}\right)$ : Cu1-N1 2.5385(28), Cu1-N5 1.995(2), Cu1-N7 2.002(3), N1-Cu1-N5 91.79(10), N1-Cu1-N7 81.15(10), N5-Cu1-N7 88.97(11), Cu1-O1 1.926(2), Cu1-O2 1.948(2), O1-O2 2.5086(33), Cu1-O1-Cu2 99.27(11), Cu1-O2-Cu2 99.03(11), O1-Cu1-O2 80.70(10), Cu1-O1-H1A 104(3), Cu2-O1-H1A 109(3), Cu1-O2-H2A 105(3), Cu2-O2-H2A 106(3), Cu1-Cu2 2.9456(6), Cu2-N9 2.5332(28), Cu2-N13 2.010(3), Cu2-N15 1.995(3), Cu2-O1 1.939(2), Cu2-O2 1.925(2), N9-Cu2-N13 80.20(10), N9-Cu2-N15 93.60(10), N13-Cu2-N15 88.12(11), O1-Cu2-O2 80.96(10), N3-O1 2.853(4), N11-O2 2.0161(15). 


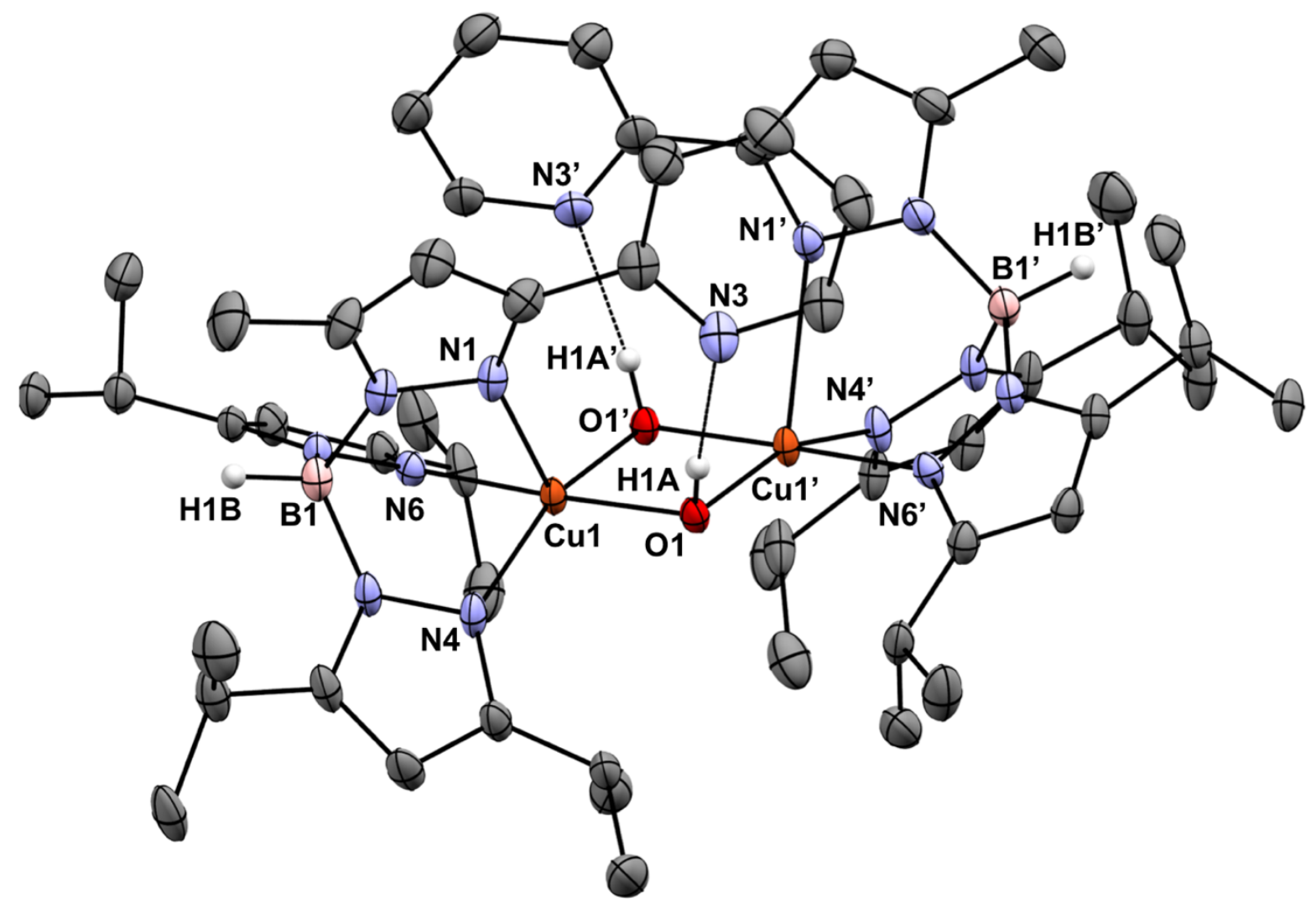

Figure S18. X-ray structure of $\left[{ }^{\mathrm{pyMe}} \mathrm{TpCu}\right]_{2}(\mu-\mathrm{OH})_{2}(\mathbf{6 c})(\mathrm{CCDC} 1890617)$. All but selected $\mathrm{H}$ atoms and DCM solvate molecules are omitted. The thermal ellipsoids are represented at the $50 \%$ probability level. Selected bond distances $(\AA)$ and angles $\left(^{\circ}\right)$ : Cu1-N1 2.4999(26), Cu1-N4 2.014(3), Cu1-N6 2.017(2), N1Cu1-N4 83.06(10), N1-Cu1-N6 91.43(9), N4-Cu1-N6 87.32(9), Cu1-O1 1.9366(19), Cu1-O1' 1.950(2), O1-O1' 2.5430(39), Cu1-O1-Cu1' 98.25(9), O1-Cu1-O1' 81.75(9), Cu1-O1-H1A 106(2), Cu1-O1'-H1A' 100(2), Cu1-Cu1’2.9384(7), N3-(H)O1 2.849(3). 


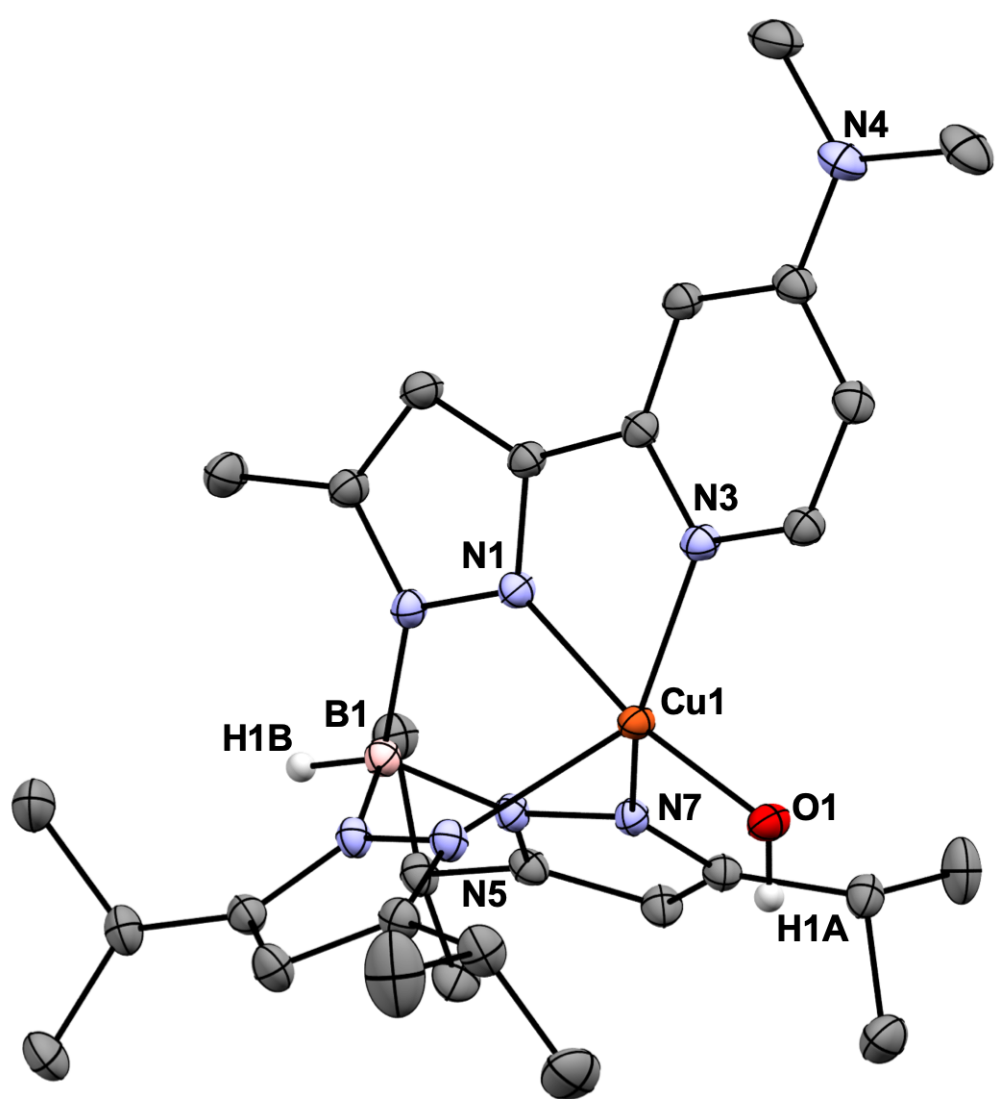

Figure S19. X-ray structure of ${ }^{\mathrm{DMAPMe}} \mathrm{TpCuOH}$ (6e) (CCDC 1890618). All but selected $\mathrm{H}$ atoms and DCM solvate molecules are omitted. The thermal ellipsoids are represented at the $50 \%$ probability level. Selected bond distances $(\AA)$ and angles $\left(^{\circ}\right)$ : Cu1-N1 1.942(2), Cu1-N3 2.087(2), Cu1-N5 2.234(3), Cu1N7 2.153(3), Cu1-O1 1.870(2) N1-Cu1-N3 76.80(6), N1-Cu1-N5 84.69(7), N1-Cu1-N7 85.64(6), N5Cu1-N7 85.64(6), N1-Cu1-O1 169.32(5), N3-Cu1-O1 92.65(6), N5-Cu1-O1 103.71(8), N7-Cu1-O1 101.65(5), Cu1-O1-H1A 107.5(14), C1-C4-N3 112.10(12). 


\section{REFERENCES}

(1) Kitajima, N.; Fujisawa, K.; Fujimoto, C.; Moro-oka, Y., Preparations of Copper(I) Complexes Ligated with with Novel Hindered Polypyrazolylborates. Chem. Lett. 1989, 18, 421-424.

(2) Chia, L. M. L.; Radojevic, S.; Scowen, I. J.; McPartlin, M.; Halcrow, M. A., Steric control of the reactivity of moderately hindered tris(pyrazolyl)borates with copper(II) salts. J. Chem. Soc. Dalt. Trans. 2000, 133-140.

(3) Kitajima, N.; Fujisawa, K.; Fujimoto, C.; Morooka, Y.; Hashimoto, S.; Kitagawa, T.; Toriumi, K.; Tatsumi, K.; Nakamura, A., A New Model for Dioxygen Binding in Hemocyanin. Synthesis, Characteriztion, and Molecular Structure of the $\mu-\eta^{2}: \eta^{2}$ Peroxo Dinuclear Copper(II) Complexes, $\left[\mathrm{Cu}\left(\mathrm{HB}\left(3,5-\mathrm{R}_{2} \mathrm{pz}\right)_{3}\right)\right]_{2}\left(\mathrm{O}_{2}\right)(\mathrm{R}=i-\mathrm{Pr}$ and $\mathrm{Ph})$. J. Am. Chem. Soc. 1992, 114, 1277-1291.

(4) Evans, D. F., The Determination of the Paramagnetic Susceptibility of Substances in Solution by Nuclear Magnetic Resonance. J. Chem. Soc. 1959, 2003-2005.

(5) Ghosh, P.; Ding, S.; Chupik, R. B.; Quiroz, M.; Hsieh, C.-H.; Bhuvanesh, N.; Hall, M. B.; Darensbourg, M. Y. A., A Matrix of Heterobimetallic Complexes for Interrogation of Hydrogen Evolution Reaction Electrocatalysts. Chem. Sci. 2017, 8, 8291-8300.

(6) Bain, G. A.; Berry, J. F., Diamagnetic Corrections and Pascal's Constants. J. Chem. Educ. 2008, $85,532-536$.

(7) Bissantz, C.; Grether, U.; Hebeisen, P.; Kimbara, A.; Liu, Q.; Nettekoven, M.; Prunotto, M.;

Roever, S.; Rogers-Evans, M.; Schulz-Gasch, T.; Ullmer, C.; Wang, Z.; Yang, W. Novel Pyridine Derivatives. U.S. Patent 20,120,316,147, December 13, 2012.

(8) Yu, W. S.; Cheng, C. C.; Cheng, Y. M.; Wu, P. C.; Song, Y. H.; Chi, Y.; Chou, P. T., ExcitedState Intramolecular Proton Transfer in Five-Membered Hydrogen-Bonding Systems: 2-Pyridyl Pyrazoles. J. Am. Chem. Soc. 2003, 125, 10800-10801.

References for Crystal Structure Refinements:

(a) Sheldrick, G. M., Crystal Structure Refinement with SHELXL. Acta Cryst. 2015, C71, 3-8.

(b) Hubschle, C. B.; Sheldrick, G. M.; Dittrich, B., ShelXle: a Qt graphical user interface for SHELXL. J. Appl. Cryst. 2011, 44, 1281-1284.

(c) Bruker (2016). APEX3, SADABS, SAINT, SHELXTL, XCIF, XPREP. Bruker AXS, Inc., Madison, Wisconsin, USA.

(d) C. F. Macrae, I. J. Bruno, J. A. Chisholm, P. R. Edgington, P. McCabe, E. Pidcock, L. RodriguezMonge, R. Taylor, J. van de Streek and P. A. Wood, Mercury CSD 2.0 - new features for the visualization and investigation of crystal structures. J. Appl. Cryst. 2008, 41, 466-470.

(e) Spek, A. L., PLATON SQUEEZE: a tool for the calculation of the disordered solvent contribution to the calculated structure factors, Acta Cryst. 2015, C71, 9-18. 